\title{
On jet substructure methods for signal jets
}

\author{
Mrinal Dasgupta, ${ }^{a}$ Alexander Powling ${ }^{b}$ and Andrzej Siodmok ${ }^{c, d}$ \\ ${ }^{a}$ Consortium for Fundamental Physics, School of Physics $\&$ Astronomy, University of Manchester, \\ Oxford Road, Manchester M13 9PL, U.K. \\ ${ }^{b}$ School of Physics \& Astronomy, University of Manchester, \\ Oxford Road, Manchester M13 9PL, U.K. \\ ${ }^{c}$ Institute of Nuclear Physics, Polish Academy of Sciences, \\ ul. Radzikowskiego 152, 31-342 Kraków, Poland \\ ${ }^{d} \mathrm{CERN}, \mathrm{PH}-\mathrm{TH}$, \\ CH-1211 Geneva 23, Switzerland \\ E-mail: Mrinal.Dasgupta@manchester.ac.uk, \\ alexander.powling@hep.manchester.ac.uk, andrzej@cern.ch
}

ABStRACT: We carry out simple analytical calculations and Monte Carlo studies to better understand the impact of QCD radiation on some well-known jet substructure methods for jets arising from the decay of boosted Higgs bosons. Understanding differences between taggers for these signal jets assumes particular significance in situations where they perform similarly on QCD background jets. As an explicit example of this we compare the Ysplitter method to the more recently proposed Y-pruning technique. We demonstrate how the insight we gain can be used to significantly improve the performance of $\mathrm{Y}$-splitter by combining it with trimming and show that this combination outperforms the other taggers studied here, at high $p_{T}$. We also make analytical estimates for optimal parameter values, for a range of methods and compare to results from Monte Carlo studies.

KEYwords: QCD Phenomenology, Jets

ARXIV EPRINT: 1503.01088 


\section{Contents}

1 Introduction 2

2 Results for plain jet mass 5

2.1 Initial state radiation 6

2.2 Final state radiation $\quad 9$

$\begin{array}{lll}2.3 & \text { Non-perturbative contributions } & 10\end{array}$

$\begin{array}{lll}3 & \text { Trimming } & 11\end{array}$

3.1 Lowest order result 11

$\begin{array}{ll}3.2 & \text { Initial state radiation } \\ \end{array}$

$\begin{array}{lll}3.3 & \text { Final state radiation } & 15\end{array}$

$\begin{array}{ll}3.4 & \text { Non-perturbative contributions } \\ \end{array}$

4 Pruning and mMDT $\quad 20$

4.1 Pruning 20

$\begin{array}{lll}4.2 & \text { Modified mass drop tagger } & 21\end{array}$

4.3 Non-perturbative effects and MC results 23

$5 \quad$ Y-pruning and Y-splitter $\quad 24$

5.1 Y-pruning 25

$\begin{array}{lll}5.2 & \text { Y-splitter } & 27\end{array}$

$6 \quad$ Y-splitter with trimming $\quad 30$

7 Optimal parameter values $\quad 33$

7.1 mMDT 34

$\begin{array}{ll}7.2 & \text { Pruning and Y-pruning }\end{array}$

$\begin{array}{lll}7.3 & \text { Trimming } & 39\end{array}$

8 Conclusions 43

A Angular integration for FSR 46

B Fixed-order results vs parton showers for FSR corrections 47 


\section{Introduction}

In recent years the detailed study and analysis of the internal structure of hadron jets has become an area of very active investigation. The principle reason for such high interest has been in the context of Higgs boson and new physics searches at the LHC and associated phenomenology. Due to the large ( $\mathrm{TeV}$ scale) transverse momenta that can be accessed at the LHC, electroweak scale particles, such as the Higgs boson, can be directly produced with large boosts. Alternatively the decay of yet undiscovered heavy new particles to comparatively light standard model particles, such as top quarks or $W / Z$ bosons, would also result in the production of boosted particles whose decay products would consequently be collimated. This in turn means that rather than producing multiple resolved jets, a significant fraction of the time the decay products are encompassed in a single fat jet. Understanding the substructure of such jets therefore becomes crucial in the context of discriminating between jets originating from QCD background and those originating from signal processes involving e.g. Higgs production and its hadronic decay.

Though pioneering studies were carried out by Seymour several years ago [1], and the Y-splitter method for tagging $\mathrm{W}$ bosons was subsequently introduced in ref. [2] over a decade ago, the revival of interest in jet substructure is relatively recent and owes essentially to seminal work by Butterworth, Davison, Rubin and Salam [3]. These authors revealed the power of substructure analyses by studying the discovery potential for a light Higgs boson $\left(M_{H} \approx 120 \mathrm{GeV}\right)$ in the process $p p \rightarrow W / Z, H$ with Higgs decay to $b \bar{b}$. They demonstrated that exploiting the boosted regime and applying jet substructure methods, specifically a mass-drop and filtering analysis, was sufficient to turn what was previously regarded as an unpromising channel into one of the best channels for Higgs discovery at the LHC. Several other applications followed, dedicated to new physics searches as well as top and $W / Z$ boson tagging, and numerous substructure techniques are now in existence and being commonly employed in experimental analyses both in the context of QCD measurements as well as for searches [4-14]. For the original articles introducing a selection of some of these methods we refer the reader to refs. [15-25] while comprehensive reviews of the field and further studies are available in refs. [26-29].

Most recently research has started to emerge [30-33] which aims at enhancing our understanding of jet substructure methods via the use of analytical calculations that, where possible, lend greater insight and provide powerful complementary information to that available purely from traditional Monte Carlo (MC) based investigations of jet substructure. In ref. [31] in particular, resummed results were provided for jet mass distributions for QCD background jets after the application of a variety of jet substructure methods (that we shall collectively refer to as 'taggers') and detailed comparisons to MC studies were carried out. Jet mass distributions were examined for the case of trimmed [21] and pruned jets [19, 20] as well as for jets obtained after the application of the mass-drop tagger [3].

One of the main aims of ref. [31] was to better understand how aspects of tagger definition and design may interplay with QCD dynamics to dictate the performance of taggers as reflected by their action on background jets. The improved analytical understanding that was achieved led to a better appreciation of the role of tagger parameters (including the 
discovery of the apparent redundancy of the mass-drop parameter $\mu$ in the mass-drop tagger [3]). The analytical studies also paved the way for improvement of theoretical properties of taggers. Examples of improvements that were suggested or made in ref. [31] included the design of taggers with a perturbative expansion more amenable to resummation as for the modified mass-drop (mMDT) as well as removing undesirable tagger features as for the case of pruning via the Y-pruning modification. Subsequent work has also demonstrated how an analytical understanding of the action of taggers on QCD background can be exploited to construct valuable new tools such as the soft drop technique introduced in ref. [33].

While thus far there has been heavy focus on taggers applied to QCD background, until now radiative effects for signal jets have not been investigated in the same level of analytical detail for many commonly used substructure methods. Detailed analytical calculations have however been performed to study the action of filtering for $H \rightarrow b \bar{b}$ [34] and for N-subjettiness [35], while the role of QCD radiation in the context of template tagging was discussed in ref. [23].

We first observe that it is common to study high $p_{T}$ signal jets in some relatively narrow mass window of width $\sim \delta M$ around the mass, $M$ of some boosted decaying heavy resonance of interest, this mass cut being a first step in tagging signal jets. One then has a situation where there are various disparate scales involved in the problem such as the (potentially) $\mathrm{TeV}$ scale transverse momenta of the fat jets, the mass $M$ of the resonance (which for our studies we can consider to be around the electroweak scale) and the width of the window $\delta M$ which for most purposes we can consider as a parameter $\sim 10 \mathrm{GeV}$. These scales are in addition, of course, to the various parameters corresponding to angular distances and energy cuts introduced by tagging and jet finding.

It is well known that in such multi-scale problems radiative corrections have the potential to produce large logarithms involving ratios of disparate scales. In the example of filtering studied in ref. [34] large logarithms in $M_{H} / \delta M$ arose from considering soft emissions, which were accompanied by collinear logarithmic enhancements in $R_{b \bar{b}} / R_{\mathrm{filt}}$, the ratio of the $b \bar{b}$ opening angle to the filtering radius. On the other hand ref. [31] observed via MC studies of the signal that for the taggers studied there (mMDT, pruning, trimming, Ypruning) the tagger performance was primarily driven by the action of taggers on QCD background, with signals not appearing to display very sizeable radiative corrections for the default parameters chosen there.

In order to better understand these apparently contrasting observations it is desirable to acquire a higher level of analytical insight into the action of taggers on signal jets. When comparing the performance of taggers one may also meet a situation where two taggers shall act essentially similarly on background jets and hence their action on signal becomes of critical significance. We shall in fact provide an explicit example of this situation later in this article. It is also of importance to understand and assess the impact of QCD radiative corrections and non-perturbative effects on tagger efficiency for signals, to ascertain what theoretical tools (fixed-order calculations, MC methods, resummed calculations or combinations thereof), should ideally be deployed to get the most reliable picture for the signal efficiency for a given tagger. 
With all the above aims in mind, here we embark on a more detailed study dedicated to signal jets. We shall focus our attention on the case of a jet arising from a boosted Higgs boson for a process such as Higgs production in association with a vector boson $p p \rightarrow W / Z, H$, with $H \rightarrow b \bar{b}$, where we will work in a narrow width approximation. For our analytical approximations we shall also typically consider highly boosted configurations i.e. those where the Higgs has transverse momentum $p_{T} \gg M_{H}$ and shall further take a fat jet with radius $R \gg \frac{M_{H}}{p_{T}}$. We shall work in a small-angle approximation throughout, though we will often consider $R \sim 1$.

We stress here that we do not intend to provide precise high-order calculations for radiative corrections to any given process but seek mainly to understand and compare the behaviour of taggers via a combination of approximate analytics and MC cross-checks. For an example of exact fixed-order calculations involving jet substructure and signal processes we refer the reader to ref. [36].

We start in the next section by analysing the case of a plain jet mass cut focussing on a mass window around the signal mass and deeming a jet to be tagged as signal if the jet mass falls within this window. We consider the impact on signal efficiency of initial state radiation (ISR), final state radiative corrections (FSR) both analytically and in MC studies. We also study the impact of non-perturbative effects (NP) with MC. The results so obtained can then provide a point of reference and comparison to judge the improvements that are offered by use of substructure taggers, which impose requirements in addition to a simple cut on mass.

Next we move to analysing jets with application of various taggers. In section 3 we study trimming at lowest order and with ISR and FSR corrections. We investigate the logarithmic structure that emerges in $M, \delta M$ and $p_{T}$ as well as in tagger parameters and compare to $\mathrm{MC}$ results where appropriate. We also study non-perturbative corrections, though purely with MC results. In section 4 we analyse along similar lines pruning and the modified mass-drop tagger (mMDT). FSR is analysed further for these taggers in appendices A and B where we also compare parton shower results to those from full leadingorder calculations i.e. those that go beyond the soft/collinear approximation.

In section 5 we study Y-pruning and the Y-splitter tagger [2]. We observe that while the action of Y-splitter on QCD background is similar to Y-pruning, the signal jet with Y-splitter is subject to severe loss of resolution due to ISR and underlying event (UE) effects. We show with MC studies that combining Y-splitter with trimming dramatically improves the signal behaviour while leaving the background largely unmodified. As a consequence we show that Y-splitter with trimming outperforms the other taggers we study here, especially at high $p_{T}$. To our mind this example further illustrates how even a relatively basic analytical understanding of all aspects of taggers (for both signal and background) can be exploited to achieve important performance gains. ${ }^{1}$

Finally we carry out analytical studies of optimal values for tagger parameters, obtained by maximising signal significance, and compare to MC results. We conclude with a summary and mention prospects for future work.

\footnotetext{
${ }^{1}$ One other recent example of simple analytical arguments, based on power counting, being used to good effect for top tagging can be found in ref. [37].
} 


\section{Results for plain jet mass}

Here we shall consider the plain jet-mass distribution for fat signal jets without the application of substructure methods, other than the imposition of a mass window $\delta M$, as previously stated. As also mentioned before, we shall consider the case of Higgs boson production in association with an electroweak vector boson $p p \rightarrow W / Z, H$ with Higgs decay to a $b \bar{b}$ quark pair and shall work in a narrow width approximation throughout. For the purposes of examining the jet substructure we shall not need to write down matrix elements for the production of the high $p_{T}$ Higgs boson and shall instead be concerned purely with the details of the Higgs decay and the resulting fat jet, as well as the impact of ISR, FSR and non-perturbative effects. Let us take a boosted Higgs boson produced with transverse momentum $p_{T} \gg M_{H}$ and purely for convenience set it to be at zero rapidity with respect to the beam direction, so that the corresponding energy is $\sqrt{p_{T}^{2}+M_{H}^{2}}$. We further consider Higgs decay into $b \bar{b}$ so that in terms of four-momenta one has $p_{H}=p_{b}+p_{\bar{b}}$. Thus the invariant mass of the Higgs can be expressed as

$$
p_{H}^{2}=M_{H}^{2}=2 p_{b} \cdot p_{\bar{b}}=2 z(1-z)\left(p_{T}^{2}+M_{H}^{2}\right)\left(1-\cos \theta_{b \bar{b}}\right),
$$

with $z$ and $1-z$ the energy fractions of the decay products and $\theta_{b \bar{b}}$ the $b \bar{b}$ opening angle, where we neglected the $b$ quark masses. Furthermore we shall consider the highly boosted regime taking $\Delta=\frac{M_{H}^{2}}{p_{T}^{2}} \ll R^{2}$ and shall systematically neglect power corrections in $\Delta$. Then from eq. (2.1), taking a small-angle approximation, we obtain the standard result:

$$
\theta_{b \bar{b}}^{2} \approx \frac{\Delta}{z(1-z)}
$$

Requiring that the Higgs decay products are contained in a single fat jet, $\theta_{b \bar{b}}^{2}<R^{2}$ thus translates into a constraint on $z$ :

$$
z(1-z)>\frac{\Delta}{R^{2}}
$$

Let us start by providing the results for the signal efficiency, $\varepsilon_{\mathrm{S}}^{(0)}$ to lowest order i.e. taking just the $H \rightarrow b \bar{b}$ decay without any radiative corrections. This can be considered as the fraction of decays that are reconstructed inside a fat jet of radius $R$. Here one has to consider the relevant Feynman amplitude for $H \rightarrow b \bar{b}$ and the full decay phase-space with an integral over the final state parton momenta. However for the fraction of decays inside the fat jet, $\varepsilon_{\mathrm{S}}^{(0)}$, we just obtain

$$
\varepsilon_{\mathrm{S}}^{(0)}=\int_{0}^{1} d z \Theta\left(R^{2}-\frac{\Delta}{z(1-z)}\right)=\sqrt{1-\frac{4 \Delta}{R^{2}}} \Theta\left(R^{2}-4 \Delta\right) \approx 1-\frac{2 \Delta}{R^{2}}+\mathcal{O}\left(\frac{\Delta}{R^{2}}\right)^{2}
$$

which is trivially in good agreement with corresponding $\mathrm{MC}$ event generator results with all ISR, FSR and non-perturbative effects turned off. The above result simply suggests, as one can easily anticipate, that with increasing boosts, i.e. smaller $\Delta$, the efficiency of reconstruction inside a single jet increases. At this lowest-order level there is of course no role for the mass-window $\delta M$ since the jet mass $M_{j}$ coincides with the Higgs mass $M_{H}$. 


\subsection{Initial state radiation}

Let us now account for the impact of initial state radiation on the jet mass distribution. We can anticipate that the impact of soft radiation may be significant here because of the fact that we require the invariant mass of the fat jet to be within $\delta M$ of the Higgs mass, with $\delta M \ll p_{T}$. This requirement imposes a constraint on real emissions, arising from ISR, that enter the jet since these contribute directly to the deviation of the jet mass from $M_{H}$. Hence one can expect that large logarithmic corrections arise as a consequence. In order to understand the structure of the logarithmic corrections that arise, we consider the process $p p \rightarrow Z H$ with the additional production of soft gluons radiated by the incoming hard partons ( $q \bar{q}$ pair). Let us start by taking a single ISR gluon which is soft i.e. has energy $\omega \ll p_{T}$. In the soft limit we can work with the eikonal approximation in which production of the ISR factorises from the Born-level hard process $p p \rightarrow Z H$. To compute the signal efficiency we shall require the jet invariant mass to be within a relatively narrow mass-window $\delta M$ of the Higgs mass:

$$
M_{H}-\delta M<M_{j}<M_{H}+\delta M .
$$

As we observed previously at lowest order (Born level) this inequality is always true since $M_{j}=M_{H}$, however with ISR it amounts to a constraint on the ISR gluon energy. Neglecting corrections of order $\delta M^{2}$ we can write:

$$
M_{j}^{2}-M_{H}^{2}=2 p_{H} \cdot k<2 M_{H} \delta M,
$$

where $p_{H}$ is the four-momentum of the Higgs (or equivalently the sum of the four-momenta of its decay products) and $k$ that of the ISR particle. Defining $\theta$ as the angle between the soft emission and the Higgs direction we can write:

$$
2 p_{H} \cdot k=2 \omega\left(\sqrt{p_{T}^{2}+M_{H}^{2}}-p_{T} \cos \theta\right) .
$$

Since we take $\Delta=M_{H}^{2} / p_{T}^{2} \ll 1$ and also use the small $\theta$ approximation, we can expand in small quantities and write the following constraint on gluon energy:

$$
\omega<\frac{2 M_{H} \delta M}{p_{T}\left(\theta^{2}+\Delta\right)} .
$$

One can express this equation in terms of standard hadron collider variables $k_{t}, \eta$ and $\phi$ defined w.r.t. the beam direction by noting simply that $\omega=k_{t} \cosh \eta$ and $\theta^{2} \approx \eta^{2}+\phi^{2}$.

We wish to examine only the leading logarithmic structure that arises from soft ISR emissions, starting with a single emission i.e. to leading order in the strong coupling. Since we are considering an emission that enters the high $p_{T}$ fat jet we are concerned with largeangle radiation from the incoming hard partons. This in turn implies that there are no collinear enhancements associated to such radiation and the resulting leading logarithmic structure ought to be single-logarithmic, arising purely from the infrared singularities in the gluon emission probability. ${ }^{2}$

\footnotetext{
${ }^{2}$ In practice this expectation is challenged by the discovery of superleading logs [39]. Since these appear at order $\alpha_{s}^{4}$ and are suppressed as $1 / N_{C}^{2}$ we do not expect them to make a significant impact on the essential arguments we make here.
} 
The gluon emission probability is in turn given by the standard two particle antenna for soft emissions off the incoming quark/anti-quark:

$$
W_{i j}=2 C_{F} \frac{\alpha_{s}}{\pi} \frac{\left(p_{i} \cdot p_{j}\right)}{\left(p_{i} \cdot k\right)\left(p_{j} \cdot k\right)}
$$

where $C_{F}=4 / 3$. Analogous to the case of jet mass distributions for hadron collider QCD jets (see for example calculations in ref. [38]) the ISR contribution can be written by integrating eq. (2.9) over the gluon emission phase space. The result can be expressed in terms of $k_{t}, \eta$ and $\phi$ and reads:

$$
\begin{aligned}
& \varepsilon_{\mathrm{S}, \mathrm{ISR}}^{(1)}=\int d z \Theta\left(z(1-z)-\frac{\Delta}{R^{2}}\right) \times \\
& \times 2 C_{F} \int \frac{d k_{t}}{k_{t}} d \eta \frac{d \phi}{2 \pi} \frac{\alpha_{s}\left(k_{t}\right)}{\pi}\left(\Theta\left(\frac{2 M_{H} \delta M}{p_{T}\left(\theta^{2}+\Delta\right)}-k_{t} \cosh \eta\right)-1\right) \Theta_{\text {jet }} .
\end{aligned}
$$

The above equation contains an integral over the energy fraction of the partonic offspring involved in the Higgs decay (i.e. that over $z$ ), with a constraint that is identical to the zeroth order requirement that the hard quarks be contained in the fat jet, which is unmodified by the presence of soft ISR at leading logarithmic level, i.e. in the limit $\omega=k_{t} \cosh \eta \ll p_{T}$. The step function involving a restriction on the transverse momentum $k_{t}$ follows directly from eq. (2.8) and the subsequent arguments. Virtual corrections are incorporated via unitarity through the -1 term also in parenthesis. Lastly we have a factor $\Theta_{\text {jet }}$ that is the condition that the soft ISR is within the fat jet.

The clustering condition $\Theta_{\text {jet }}$ is in principle quite complicated since it involves recombination of three particles within the fat jet, namely the $b, \bar{b}$ and the ISR gluon. In our approximation of $\Delta \ll R^{2}$ i.e. in the limit of large boosts, we are considering a highly collimated quark pair, relative to the radius of the fat jet. One can thus ignore the effect of the finite $b \bar{b}$ opening angle as these effects contribute only terms that are relatively suppressed by powers of $\Delta$ compared to the leading term we compute. Then one only has to consider the fact that the soft ISR gluon is in the interior of the fat jet which amounts to the condition $\Theta_{\text {jet }}=\Theta\left(R^{2}-\left(\eta^{2}+\phi^{2}\right)\right)$, since we had taken the Higgs rapidity as zero.

Within the context of the current purely order $\alpha_{s}$ calculation we shall also consider the coupling as fixed at scale $p_{T}$ and ignore its running. Running coupling effects are of course important to include for leading logarithmic resummation and we shall do so for our final answers. We define $\varepsilon_{\mathrm{S} \text {,ISR }}=\varepsilon_{\mathrm{S}}^{(0)}+\varepsilon_{\mathrm{S} \text {,ISR }}^{(1)}$ and carrying out the relevant integrations with fixed coupling we get from eq. (2.10) the leading logarithmic result:

$$
\frac{\varepsilon_{\mathrm{S}, \mathrm{ISR}}}{\varepsilon_{\mathrm{S}}^{(0)}} \simeq 1-\frac{C_{F} \alpha_{s}}{\pi} R^{2} \ln \left(\frac{p_{T}^{2} R^{2}}{2 M_{H} \delta M}\right) .
$$

In order to obtain the above result starting from eq. (2.10) one can first integrate over $k_{t}$, discarding the $\cosh \eta$ accompanying factor as this will only generate subleading terms. The integral over $k_{t}$ produces the large logarithm we seek. One can then express the $\eta, \phi$ integral as one w.r.t. $\theta^{2}=\eta^{2}+\phi^{2}$ and then integrate over $\theta^{2}$ with the condition $\theta^{2}<R^{2}$. 
With neglect of subleading terms, including those which vanish with $\Delta$, one then obtains the result reported above for the quantity $\varepsilon_{\mathrm{S}, \mathrm{ISR}} / \varepsilon_{\mathrm{S}}^{(0)}$. We have however chosen to retain the formally subleading logarithmic dependence on $R$ which can become important at smaller values of $R$, e.g. $R \sim 0.4$.

Given the large single logarithms that emerge from the above approximate fixed-order calculation, it is natural to wish to attempt to resum at least the leading logarithms to all perturbative orders. This is far from a straightforward exercise. One of the main obstacles to performing a soft single log resummation, in the present context, is the presence of non-global logarithms, associated clustering logarithms [40-43] as well as superleading logarithms referred to previously. Such calculations pose a serious challenge to the current state of the art and are beyond the scope of our work.

In the absence of a complete resummed calculation one can still obtain a working estimate that can be compared to MC results, simply by exponentiating the order $\alpha_{s}$ result obtained above and by including running coupling effects.

The exponentiated result including the running of the QCD coupling is given by

$$
\frac{\varepsilon_{\mathrm{S}, \mathrm{ISR}}^{P}}{\varepsilon_{\mathrm{S}}^{(0)}} \approx \exp \left(-2 C_{F} R^{2} t\right)
$$

where we defined the single-log evolution variable

$$
\begin{aligned}
t & =\frac{1}{2 \pi} \int_{\frac{2 M_{H} \delta M}{p_{T} R^{2}}}^{p_{T}} \frac{d k_{t}}{k_{t}} \alpha_{s}\left(k_{t}\right) \\
& =\frac{1}{4 \pi \beta_{0}} \ln \frac{1}{1-2 \lambda}, \quad \lambda=\beta_{0} \alpha_{s}\left(p_{T}\right) \ln \frac{p_{T}^{2} R^{2}}{2 M_{H} \delta M},
\end{aligned}
$$

where $\beta_{0}=\frac{1}{12 \pi}\left(11 C_{A}-2 n_{f}\right)$, and we shall use $n_{f}=5$. Note that we have indicated the exponentiated result by the superfix $P$, which indicates the resummed contribution from primary emissions alone i.e. excluding secondary emissions which lead to non-global logarithms. We observe here that the perturbative calculations break down at $\lambda=1 / 2$ which corresponds to an ISR emission with $k_{t} \sim \Lambda_{\mathrm{QCD}}$, the QCD scale. This translates into a value of $\delta M$ which is

$$
\delta M_{N P}=\frac{\Lambda_{\mathrm{QCD}} p_{T}}{2 M_{H}}
$$

where $\delta M_{N P}$ is the point of breakdown for perturbative calculations. Taking a value of $\Lambda_{\mathrm{QCD}}=1 \mathrm{GeV}$ for $p_{T}=3 \mathrm{TeV}$ we can deduce that we should not use perturbative results below $\delta M \sim 12 \mathrm{GeV}$.

Although we have emphasised that our estimate of the ISR corrections to the signal efficiency are incomplete, even to leading logarithmic accuracy, it is nevertheless of interest to compare to $\mathrm{MC}$ event generators. This is at least in part because $\mathrm{MC}$ generators themselves do not attain full single logarithmic accuracy and certainly exclude superleading logarithms. They do however contain a number of effects that would be formally subleading from the viewpoint of our calculation but could be of non-negligible significance numerically. Hence while we do not intend to make a detailed quantitative comparison we do expect to find qualitative similarities with MC results. 

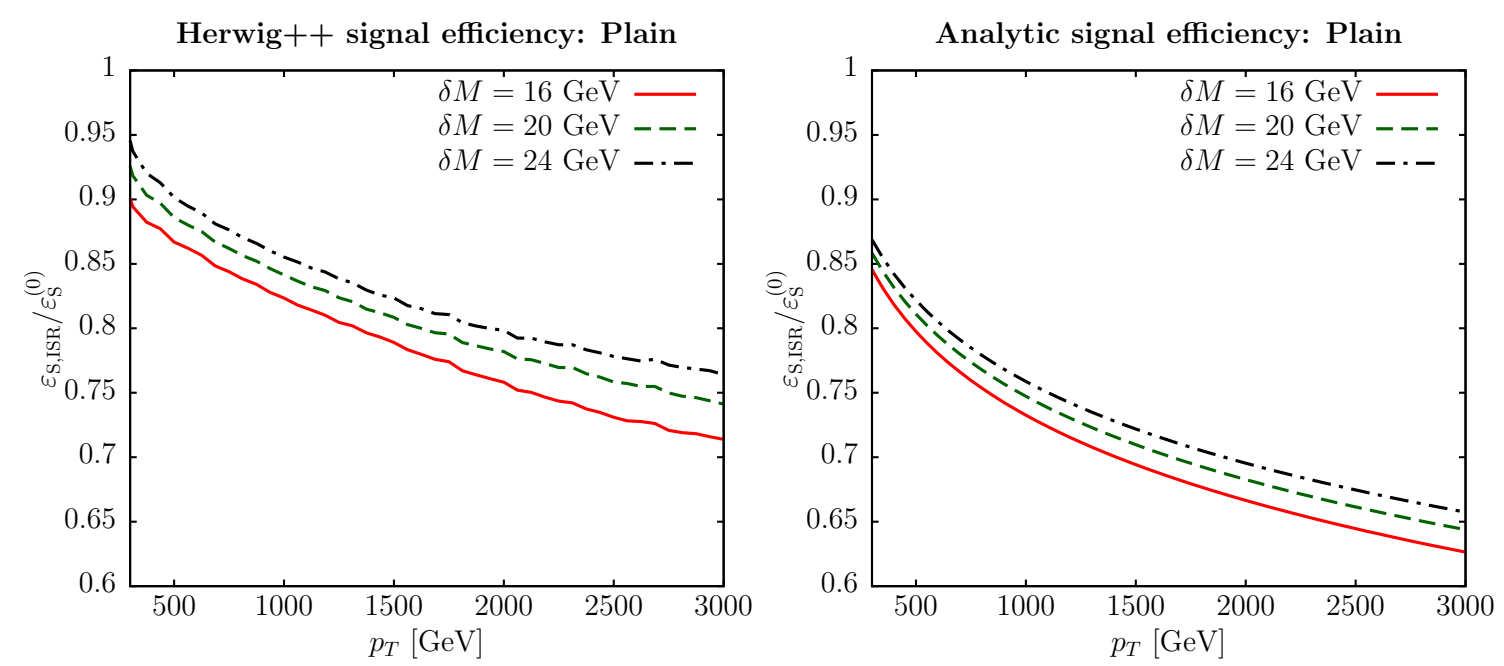

Figure 1. Comparison of MC (left) and analytic (right) eq. (2.12) tagging efficiencies for a range of mass windows as a function of a generator level cut on minimum jet transverse momentum $p_{T}$. This result has been generated using Herwig ++2.7 .0 for $p p \rightarrow Z H$ at $14 \mathrm{TeV}$ with the $\mathrm{Z}$ decaying leptonically and $H \rightarrow b \bar{b}$, setting $M_{H}=125 \mathrm{GeV}$. We have tagged the signal jet as the highest $p_{T}$ Cambridge/Aachen jet with $R=1$. In this figure we have generated events at parton level with ISR only and divided out the contribution due to the lowest order result in both panels for clarity.

To make this comparison, we generate $p p \rightarrow Z H$ events at $14 \mathrm{TeV}$ using Herwig ++ 2.7.0 with the UE-EE-5-MRST tune [46] and constrain the Higgs and Z boson to decay hadronically and leptonically respectively.

Each generated event is directly handed over to the Rivet package [47], which implements our analyses. We tag the signal jet as the highest $p_{T}$ Cambridge/Aachen $[48,49]$ jet with $R=1$ as implemented in FastJet package [50] and plot the fraction of jets which lie in mass in the window $M_{H} \pm \delta M$ as a function of a generator level cut on jet transverse momentum for three separate values of $\delta M$. To make a comparison with our ISR results we omit FSR and non-perturbative corrections including hadronisation and underlying event (UE) corrections, switching them off for the MC results. The resulting comparison is shown in figure 1, where results are displayed for the ratio of the signal efficiency to the lowest order result. We observe that the signal efficiency, from MC, decreases with transverse momentum for all values of $\delta M$ as indicated by our exponentiated result eq. (2.12). By making the mass window wider, i.e. choosing a larger $\delta M$, one of course obtains a smaller Sudakov suppression and hence a larger efficiency but starts to lose the association with a well defined signal peak.

\subsection{Final state radiation}

For the case of plain jet-mass we would expect that the correction due to final state radiation can be neglected in our region of interest where $p_{T} \gg M_{H}$ or equivalently $\Delta \ll 1$. Physically FSR is associated to the $b \bar{b}$ dipole originating from Higgs decay. It is captured within the fat jet as long as the FSR gluons are not radiated at angles beyond those corresponding to the jet radius $R$. Due to angular ordering however, we would expect that 
most of the FSR radiation from the $b$ quarks is emitted at angles smaller than $\theta_{b \bar{b}}^{2}$. In this limit the final state emission is always recombined inside the fat jet. To be more precise, large-angle radiation beyond the jet-radius $R$ is cut-off by the ratio of the dipole size $(b \bar{b}$ opening angle, given by $\Delta /(z(1-z))$, to the jet radius squared. Upon integration over $z$ such corrections translate into terms varying at most as $\Delta \ln \Delta$, which we shall neglect as they vanish with $\Delta$. We have verified this with $\mathrm{MC}$ and find that for sufficiently large transverse momenta, the correction due to final state radiation is of negligible magnitude $\mathcal{O}(0.5 \%)$ when compared to ISR $\mathcal{O}(20 \%)$ for $R=1$ and $p_{T} \gtrsim 500 \mathrm{GeV}$. We shall need to consider FSR more carefully when it comes to analysing the taggers in future sections.

\subsection{Non-perturbative contributions}

In order to get a complete picture of the physical effects that dictate the signal efficiency we also need to study how the signal efficiency changes after including non-perturbative effects such as hadronisation and underlying event (UE). In order to estimate those effects we used Herwig ++2.7 .0 with improved modeling of underlying event [51] and the most recent UE-EE-5-MRST tune [46] which is able to describe the double-parton scattering cross section [52] and underlying event data from $\sqrt{s}=300 \mathrm{GeV}$ to $\sqrt{s}=7 \mathrm{TeV}$. It can readily be anticipated that the underlying event effect in particular will significantly degrade the mass peak and hence lead to a loss of signal.

For this study, we consider all final state hadrons to be stable, therefore we switch off the decay handler module in Herwig++. In doing so, we eliminate the chance of $b$ flavour hadrons decaying into invisible particles such as neutrinos. If one were to include hadronic decay via invisible particles, one notices a universal reduction in signal efficiency for each tagger due to a loss of signal mass resolution. This is particularly important for the jets formed from the decay $H \rightarrow b \bar{b}$ as compared to $W / Z$ jets because these electroweak bosons instead couple strongly to light quarks. For further information on experimental techniques to mitigate the impact of these particular sources of missing transverse energy, see for example [53]. We also assume a $b$-tagging efficiency of $100 \%$ which is sufficient for a relative comparison of tagger performance and behaviour. The reader is referred to ref. [3] for a discussion on the impact of $b$-tagging efficiency on signal significance.

In figure 2 we see how non-perturbative effects such as hadronisation and underlying event affect the signal efficiency when using plain jets tagged with $\delta M=16 \mathrm{GeV}$. One immediately notices that whilst hadronisation has a more moderate effect on the signal efficiency, which however increases with $p_{T}$ (more precisely like $\sqrt{p_{T}}$, see ref. [54]), the dominant contribution comes from underlying event contamination which reduces the efficiency at $p_{T}=3 \mathrm{TeV}$ from about 60 percent to around 20 percent. This implies simply that one needs to consider removal of the UE for efficient tagging, which we shall discuss when we come to the boosted object taggers. We have also presented results here for $R=1$ and the averaged UE contribution to the squared jet mass varies as $R^{4}$ [54]. Thus working with smaller $R$ jets one may expect this contribution to be less significant. One should of course consider also the presence of considerable pile-up contamination, which we do not treat in this paper (see [55-57] for discussion of pileup subtraction techniques), but to which the plain jet mass will also be very susceptible. 


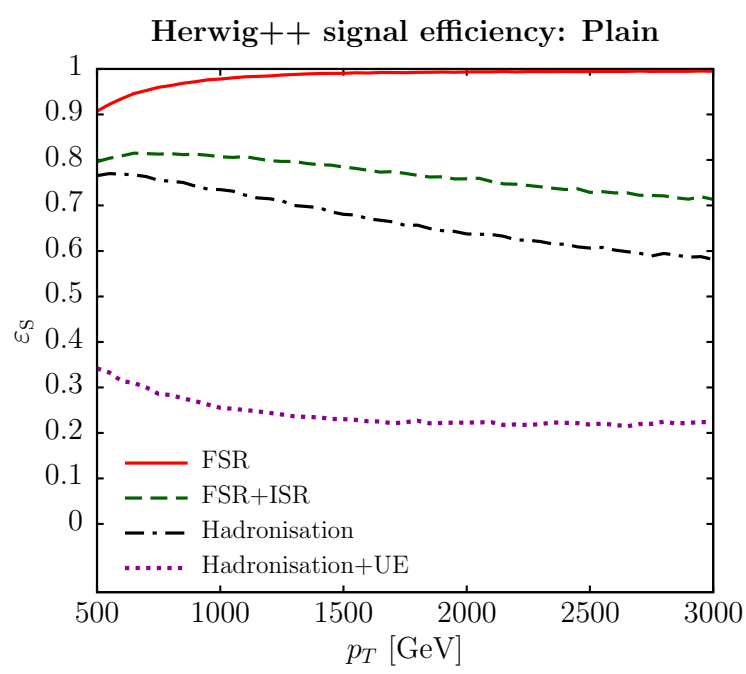

Figure 2. An MC study of the impact of hadronisation and underlying event on the signal efficiency for a plain jet mass cut as a function of the minimum jet transverse momentum. One can see the sizeable impact of both hadronisation and especially underlying event on the signal efficiency in the window $\delta M=16 \mathrm{GeV}$. Details of the generation are as in figure 1 but now we also include FSR at parton level.

For now it is evident (as is well known) that the plain jet, with a mass window cut, is not a useful option from the viewpoint of tagging signal jets due principally to effects such as ISR, UE and pile-up contamination. It however provides a reference point for the discussions to follow.

\section{Trimming}

Trimming [21] takes all the particles in a jet defined with radius $R$ and reclusters them into subjets using a new jet definition with radius $R_{\text {trim }}<R$. It retains only the subjets which carry a minimum fraction $f_{\text {cut }}$ of the original jet transverse momentum $p_{T}^{\text {(subjet) }}>f_{\text {cut }} \times p_{T}^{(\text {jet })}$ and discards the others. The final subjets are merged to form the trimmed jet.

It is standard to use the Cambridge-Aachen (C/A) jet algorithm [48, 49] for substructure studies with trimming (and other taggers) and this is what we shall employ here.

\subsection{Lowest order result}

Compared to the plain jet mass, trimming already has a more interesting structure even without considering any additional radiation. If the opening angle between the $b \bar{b}$ pair is less then $R_{\text {trim }}$ then trimming is inactive. However, if the angle is greater than $R_{\text {trim }}$, one removes the softer particle if its energy fraction is below $f_{\text {cut }}$. The result for signal efficiency is therefore given by an integral over $z$ which can be expressed as,

$$
\varepsilon_{\mathrm{S}}^{(0)}=\int_{0}^{1} d z\left(1-\Theta\left(f_{\text {cut }}-\min [z, 1-z]\right) \Theta\left(\frac{\Delta}{R_{\text {trim }}^{2}}-z(1-z)\right)\right) .
$$


Strictly we should also have written above the condition for the hard prongs to be inside the fat jet as we did for the plain jet case. However since this condition only results in terms varying as $\Delta / R^{2}$ we shall neglect it here, consistent with our approximation $\Delta / R^{2} \ll 1$.

The subtracted term in the above equation represents the removal of any prong that has energy fraction below $f_{\text {cut }}$, in the region where trimming is active.

Evaluating the integral in eq. (3.1) gives the result

$$
\begin{aligned}
& \varepsilon_{\mathrm{S}}^{(0)}=\left(1-2 f_{\text {cut }}\right) \Theta\left(1-2 f_{\text {cut }}\right)+\sqrt{1-\frac{4 \Delta}{R_{\text {trim }}^{2}}} \Theta\left(\frac{1}{4}-\frac{\Delta}{R_{\text {trim }}^{2}}\right) \Theta\left(f_{\text {cut }}-\frac{1}{2}\right)+ \\
& +\left(2 f_{\text {cut }}-1+\sqrt{1-\frac{4 \Delta}{R_{\text {trim }}^{2}}}\right) \Theta\left(\frac{1}{4}-\frac{\Delta}{R_{\text {trim }}^{2}}\right) \Theta\left(\frac{1}{2}-f_{\text {cut }}\right) \Theta\left(f_{\text {cut }}-\frac{1}{2}\left(1-\sqrt{1-\frac{4 \Delta}{R_{\text {trim }}^{2}}}\right)\right)
\end{aligned}
$$

For now we shall consider values of $f_{\text {cut }}$ that are standard in trimming analyses and therefore are considerably smaller than 0.5 . For such choices of $f_{\text {cut }}$ the second term in eq. (3.2), which requires $f_{\text {cut }}>1 / 2$, clearly does not contribute. While the result in eq. (3.2) is general, let us for illustrative purposes consider values of $R_{\text {trim }}$ not too small, such that $\Delta / R_{\text {trim }}^{2} \ll 1$. Then eq. (3.2) implies a transition point at $\Delta \simeq f_{\text {cut }} R_{\text {trim }}^{2}$, which translates to a transition point at $p_{T}=M_{H} /\left(\sqrt{f_{\text {cut }}} R_{\text {trim }}\right)$. We remind the reader that the mass distribution for background jets also had transition points at $M_{j}^{2} / p_{T}^{2}=$ $f_{\text {cut }} R^{2}$ and $M_{j}^{2} / p_{T}^{2}=f_{\text {cut }} R_{\text {trim }}^{2}$ [31, 32]. The latter transition point is coincident with that reported above for the signal and corresponds to the minimal jet mass that can be obtained with trimming for a splitting with opening angle $R_{\text {trim}}$. As one increases $p_{T}^{2}$ beyond $M_{j}^{2} /\left(f_{\text {cut }} R_{\text {trim }}^{2}\right)$ the background distribution starts to grow due to the onset of a double logarithmic behaviour so the mistag rate increases.

Below this value of $p_{T}$ the signal efficiency is given by $1-2 f_{\text {cut }}$ and is therefore $p_{T}$ independent while above it one obtains $\sqrt{1-\frac{4 \Delta}{R_{\text {trim }}^{2}}}$ and hence acquires a $p_{T}$ dependence. We remind the reader that these results apply specifically to the Higgs decay and for processes involving $W / Z$ tagging different results will be obtained. This is due to the different splitting functions involved in hadronic $W / Z$ decay.

In figure 3 we compare the signal efficiency using Herwig ++2.7 .0 for trimming applied to boosted Higgs jets with no ISR, FSR or non-perturbative effects to the analytical calculation above eq. (3.2). We generate the tagging efficiency with two different $f_{\text {cut }}$ values and for $R_{\text {trim }}=0.3$ as a function of $p_{T}$ for both MC (left) and analytics (right). One observes, as we would expect, that the MC clearly reproduces the analytic behaviour of the tagger at lowest order and, for our choice of parameters, the expected transition points at around $1320 \mathrm{GeV}$ and $1860 \mathrm{GeV}$ for $f_{\text {cut }}=0.1$ and $f_{\text {cut }}=0.05$ respectively.

\subsection{Initial state radiation}

Let us consider the action of trimming on ISR and compare to the case of the plain jet. For the plain jet we found a large logarithmic term that results in loss of signal with increasing $p_{T}$. On the other hand we would expect trimming to substantially remove ISR radiation and hence wish to check the impact on the logarithmically enhanced terms that emerge from considering soft ISR. The key difference with the plain jet case is that when the angle 

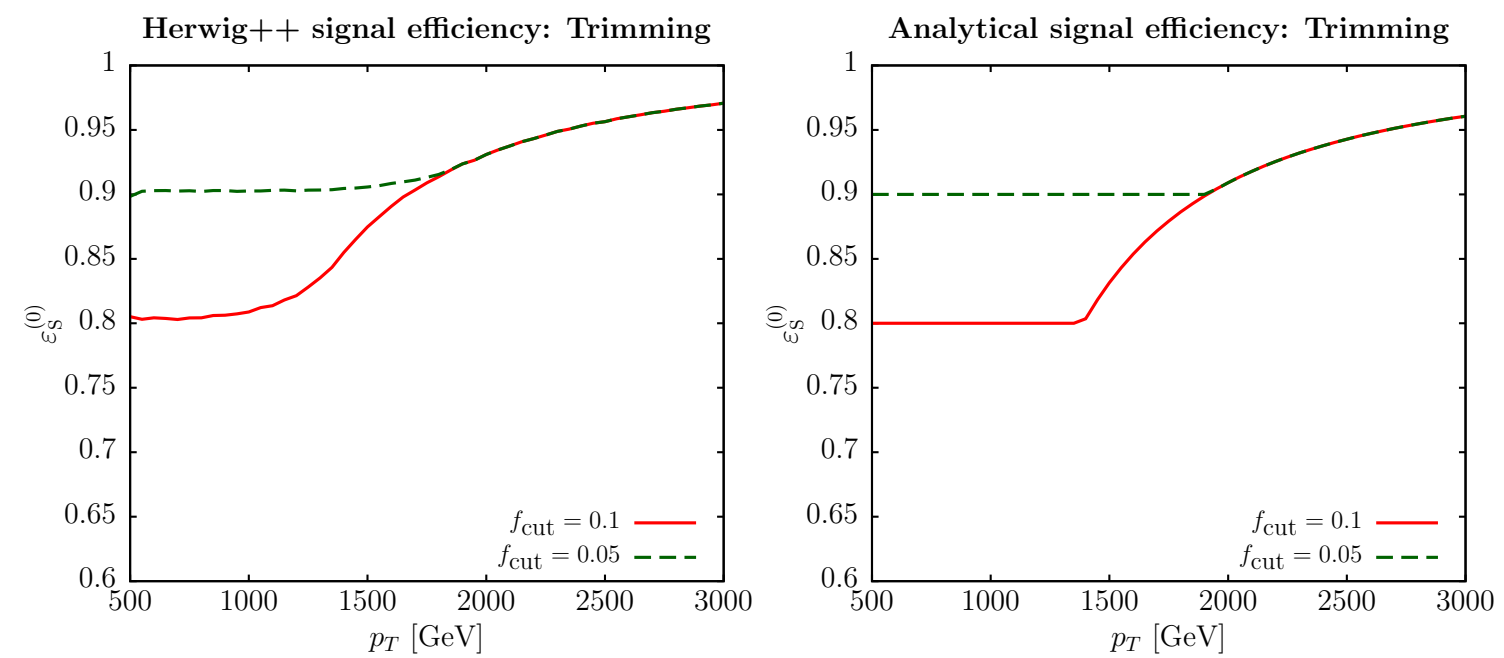

Figure 3. Comparison of MC (left) with the analytic result eq. (3.2) (right) with $R_{\text {trim }}=0.3$ for the tagging efficiency for two values of $f_{\text {cut }}$ as a function of generator level of jet transverse momentum. This result has been generated using Herwig ++2.7 .0 at parton level with no additional radiation for $H \rightarrow b \bar{b}$ jets. We note that the location of the transition points are reproduced by MC.

between the ISR gluon and the jet axis exceeds $R_{\text {trim }}$ the soft gluon is retained only if it has $k_{t} / p_{T}$ greater than $f_{\text {cut }}$, where $k_{t}$ is the transverse momentum of the soft gluon. If the $k_{t}$ fraction is below $f_{\text {cut }}$ the ISR emission is removed by trimming, thus not contributing to the jet mass, and hence in this region there is a complete cancellation with virtual corrections. Alternatively, if the ISR falls into the trimming radius, we always retain the emission, much like the plain jet case. These constraints on real emission can be expressed as:

$$
\begin{aligned}
\Theta_{\text {ISR }, \text { trim }}= & \Theta\left(\theta^{2}-R_{\text {trim }}^{2}\right)\left(\Theta\left(x-f_{\text {cut }}\right) \Theta\left(\frac{2 M_{H} \delta M}{p_{T}^{2}\left(\theta^{2}+\Delta\right)}-x\right)+\Theta\left(f_{\text {cut }}-x\right)\right) \\
& +\Theta\left(R_{\text {trim }}^{2}-\theta^{2}\right) \Theta\left(\frac{2 M_{H} \delta M}{p_{T}^{2}\left(\theta^{2}+\Delta\right)}-x\right)
\end{aligned}
$$

where we defined $x$ as $k_{t} / p_{T}$ and $\theta^{2}=\eta^{2}+\phi^{2}$ is the angle between the ISR gluon and the fat jet axis.

One can then repeat the calculation carried out for the plain jet mass in the previous section using the above constraint. Taking the ISR emission probability in the eikonal approximation as before, and incorporating virtual corrections we get (in a fixed-coupling approximation)

$$
\frac{\varepsilon_{\mathrm{S}, \mathrm{ISR}}}{\varepsilon_{\mathrm{S}}^{(0)}}=1+C_{F} \frac{\alpha_{s}}{\pi} \int_{0}^{1} \frac{d x}{x} d \theta^{2}\left[\Theta_{\mathrm{ISR}, \text { trim }}-1\right]
$$

We can evaluate the integrals straightforwardly and again shall discard terms that are power suppressed in $\Delta$. The result obtained has two distinct regimes: for $f_{\text {cut }}>\frac{2 M_{H} \delta M}{R^{2} p_{T}^{2}}$ one gets an answer of the form

$$
\frac{\varepsilon_{\mathrm{S}, \mathrm{ISR}}}{\varepsilon_{\mathrm{S}}^{(0)}} \approx 1-C_{F} \frac{\alpha_{s}}{\pi}\left(R^{2} \ln \frac{1}{f_{\text {cut }}}+R_{\text {trim }}^{2} \ln \left(\frac{f_{\text {cut }} p_{T}^{2} R_{\text {trim }}^{2}}{2 M_{H} \delta M}\right) \Theta\left(f_{\text {cut }}-\frac{2 M_{H} \delta M}{p_{T}^{2} R_{\text {trim }}^{2}}\right)\right),
$$


while for $f_{\text {cut }}<\frac{2 M_{H} \delta M}{R^{2} p_{T}^{2}}$, we get

$$
\frac{\varepsilon_{\mathrm{S}, \mathrm{ISR}}}{\varepsilon_{\mathrm{S}}^{(0)}} \approx 1-C_{F} \frac{\alpha_{s}}{\pi} R^{2} \ln \frac{R^{2} p_{T}^{2}}{2 M_{H} \delta M}
$$

Eqs. (3.5) and (3.6) basically tell us that for sufficiently large $f_{\text {cut }}$ i.e. above $\frac{2 M_{H} \delta M}{R^{2} p_{T}^{2}}$ one eliminates the logarithm we obtained for the plain jet mass replacing it by a less harmful $\ln 1 / f_{\text {cut }}$, provided one chooses $f_{\text {cut }}$ not too small. On the other hand for smaller $f_{\text {cut }}$ we see a transition to the logarithmic dependence seen for the plain mass. There is an additional correction term in equation eq. (3.5) that represents the region of integration with $\theta^{2}<R_{\text {trim }}^{2}$. This term vanishes as $R_{\text {trim }} \rightarrow 0$ and suggests that choosing smaller $R_{\text {trim }}$ values will result in less contamination from ISR as one may readily expect. We shall however see later, when studying FSR radiative corrections, that we cannot choose $R_{\text {trim }}^{2}$ too small, i.e. $\ll \Delta$, due to degradation of the jet due to FSR loss. If one chooses $R_{\text {trim }}^{2} \ll 1$ but of order $\Delta$, then within our small $\Delta$ approximation we can simply ignore this term. If on the other hand one chooses $R_{\text {trim }}$ to not be too small then at very high $p_{T}$ one should also consider the presence of this term, which appears only for $f_{\text {cut }}>\frac{2 M_{H} \delta M}{p_{T}^{2} R_{\text {trim }}^{2}}$. For most practical purposes, with commonly used parameter values, this term can safely be ignored. For example with $f_{\text {cut }}=0.1, R_{\text {trim }}=0.3$ and $\delta M=16 \mathrm{GeV}$, even at $p_{T}=3 \mathrm{TeV}$ it only contributes order 10 percent corrections relative to the main $\ln 1 / f_{\text {cut }}$ piece.

In principle, we should also resum the logarithms of $f_{\text {cut }}$ that are obtained with trimming. Such a resummation is however also beset by non-global and clustering logarithms and therefore highly involved. Moreover the $\ln 1 / f_{\text {cut }}$ terms also play only a modest role numerically, for typical choices of $f_{\text {cut }} \sim 0.1$ and thus their resummation is not particularly motivated on phenomenological grounds. We note that $\ln 1 / f_{\text {cut }}$ enhanced terms are also produced in corresponding calculations for QCD background [31] and were not resummed in that case either. Consequently, unlike the plain jet case, we do not exponentiate the radiative corrections to the signal efficiency for trimming, or any of the other taggers studied in this paper.

Let us then compare the main features of our simple analytical NLO approximation, augmented to include running coupling effects, to what is seen in MC event generators. In figure 4 we again compare our analytical approximations, with running coupling effects as in the plain mass case eq. (2.13), to Herwig ++2.7 .0 . For the MC studies we turn on ISR effects with boosted $H \rightarrow b \bar{b}$ jets for a range of $f_{\text {cut }}$ values, as a function of jet $p_{T}$, keeping $\delta M$ fixed at $16 \mathrm{GeV}$.

Plotting the ratio of the ISR corrected signal efficiency to the lowest order result, we can see that the approximate NLO analytic result reproduces the MC trends reasonably well. For values of $f_{\text {cut }}=0.1$ and 0.05 we do not obtain a transition over the range of $p_{T}$ values shown (transition points are expected at roughly $200 \mathrm{GeV}$ and $280 \mathrm{GeV}$, which are beyond the range shown) and none is seen in the MC plots. The behaviour over the entire plotted $p_{T}$ range is quite flat with $p_{T}$ since it depends mainly on $\ln 1 / f_{\text {cut }}$, with running coupling and uncalculated subleading effects (in the case of the $\mathrm{MC}$ results) providing the mild $p_{T}$ dependence that is seen. Instead for $f_{\text {cut }}=0.005$ we would anticipate a plain mass 

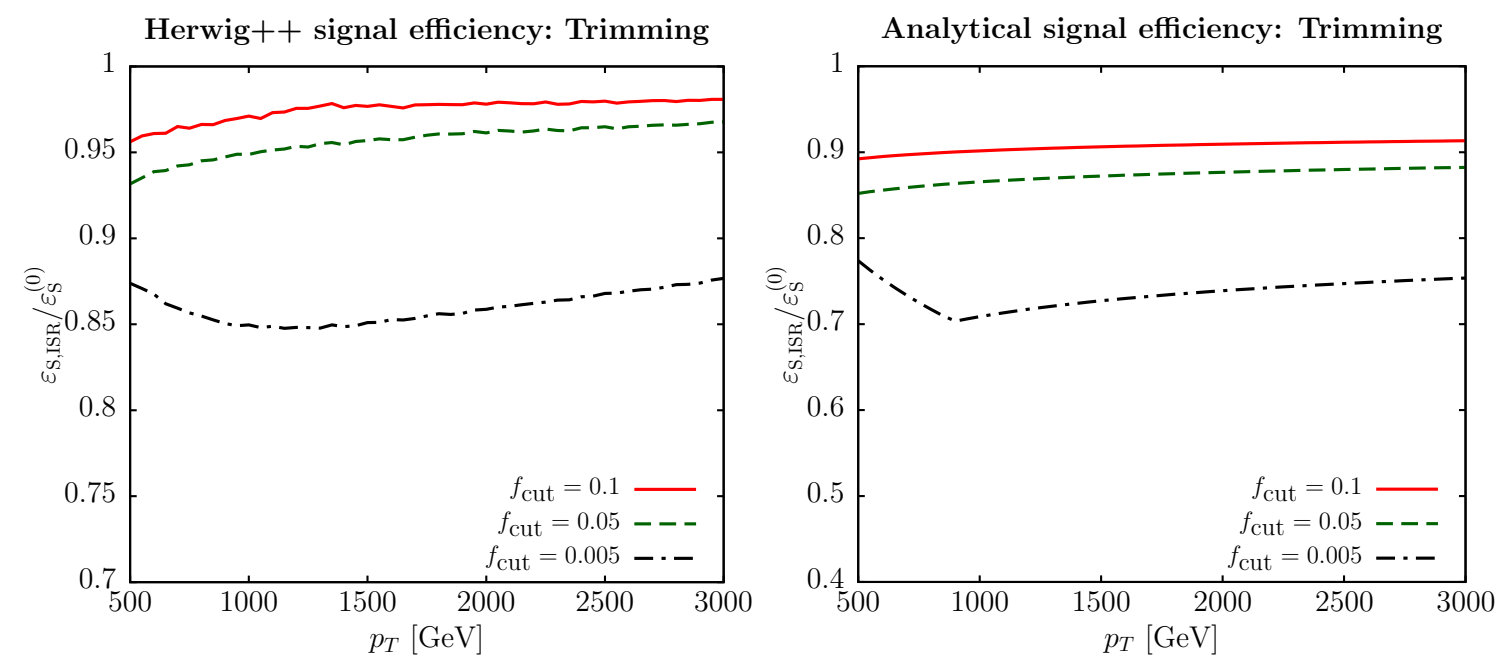

Figure 4. Comparison of MC (left) and analytic eq. (3.4) (right) trimming $\left(R_{\text {trim }}=0.3\right)$ tagging efficiencies for a range of $f_{\text {cut }}$ values as a function of a generator level cut on jet transverse momentum. This result has been generated using Herwig ++2.7 .0 [44] at parton level with ISR only for $H \rightarrow b \bar{b}$ jets, setting $M_{H}=125 \mathrm{GeV}$ and $\delta M=16 \mathrm{GeV}$. The transition points correspond to the change from plain jet mass like behaviour to a $\ln f_{\text {cut }}$ term, discussed in the main text.

like degradation of the signal efficiency until the transition at about $890 \mathrm{GeV}$ and then for higher $p_{T}$ a flatter behaviour with $p_{T}$, consistent with $\mathrm{MC}$ results. This relative flatness over a large range of $p_{T}$ is of course in contrast to the pure mass cut case.

\subsection{Final state radiation}

Let us consider the response of trimming to final state radiation. In principle there are a number of parameters to be considered, in particular $f_{\text {cut }}, \Delta$ and $R_{\text {trim }}$ as well as the mass window $\delta M$ and transverse momentum $p_{T}$. Final state radiation, when not recombined into the fat jet, results in a shift in mass which can cause the resulting jet to fall outside the mass window $\delta M$. Imposing a veto on soft FSR that degrades the jet mass results in the appearance of large logarithms whose structure we examine here. Additionally a relatively hard FSR gluon can also result in one of the primary $b$ quarks falling below the asymmetry cuts that are used in taggers, and hence loss of the signal. Such hard configurations can still come with collinear enhancements and so their role should also be considered.

In order for an FSR gluon to be removed by trimming it has to be emitted at an angle larger than $R_{\text {trim }}$ w.r.t. both the hard primary partons. In addition its energy, expressed as a fraction of the fat jet energy, must fall below the $f_{\text {cut }}$ cut-off. Lastly for the resulting jet to be retained, the consequent loss in mass must be less than $\delta M$.

One can therefore write the following result for real emission contributions, valid in the soft limit where the gluon energy $\omega \ll p_{T}$ :

$$
\varepsilon_{\mathrm{S}, \mathrm{FSR}, \mathrm{REAL}}^{(1)}=C_{F} \frac{\alpha_{s}}{\pi} \int_{f_{\text {cut }}}^{1-f_{\text {cut }}} d z \int \frac{d \omega}{\omega} \frac{d \Omega}{2 \pi} \frac{(b \bar{b})}{(b k)(\bar{b} k)} \Theta_{\text {trim }}^{\mathrm{FSR}} \Theta\left(f_{\text {cut }}-\frac{\omega}{p_{T}}\right) \Theta\left(\delta M-\left(M_{H}-M_{j}\right)\right),
$$




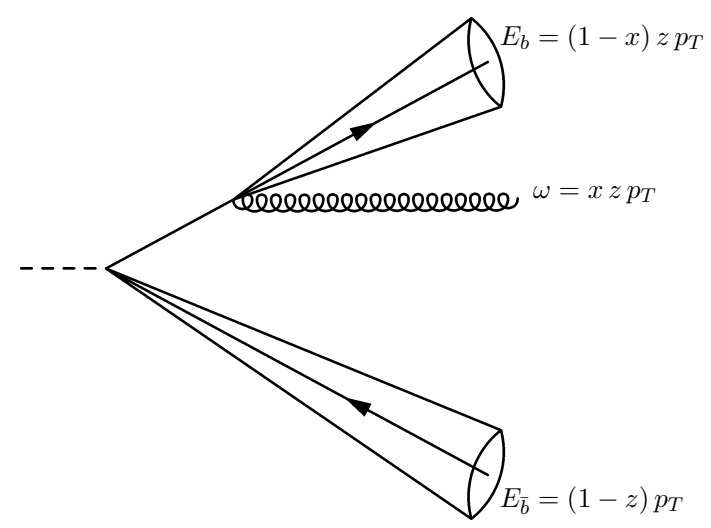

Figure 5. A configuration which contributes to the FSR correction to trimming signal efficiency in the region $R_{\text {trim }} \ll \theta_{b \bar{b}}$. Here gluon $k$ is emitted collinear to the $b$ quark with momentum fraction $x$ outside a cone with radius $R_{\text {trim }}$.

where $d \Omega$ is the solid angle element for the emitted gluon and the spatial distribution of radiation has been expressed in terms of the standard antenna pattern with the notation $(i j)=1-\cos \theta_{i j}$. The condition $\Theta_{\text {trim }}^{\mathrm{FSR}}$ simply represents that the angular integration should be carried out over the region where trimming is active i.e. when the emitted gluon makes an angle larger than $R_{\text {trim }}$ with both $b$ and $\bar{b}$. Moreover there is an additional step function that constrains the energy fraction to be below $f_{\text {cut }}$ and the factor $\Theta\left(\delta M-\left(M_{H}-M_{j}\right)\right)$ represents the constraint on real emissions due to the mass window $\delta M$.

There are three distinct regimes one can consider according to the value of $R_{\text {trim }}$. Firstly when one has $R_{\text {trim }} \ll \theta_{b \bar{b}}$ then one can expect a collinear enhancement with a logarithm in $R_{\text {trim }}$, that accompanies a soft logarithm arising out of the $\delta M$ constraint. This should be the most singular contribution one obtains for trimming so we analyse it in more detail below. In the region where $R_{\text {trim }} \sim \theta_{b \bar{b}}$ on the other hand there will be no collinear enhancement and one obtains a pure soft single logarithm. In this region trimming is similar to pruning and the mMDT as far as FSR is concerned, and we shall comment on the results in somewhat more detail in the next section. Finally in the region where $R_{\text {trim }} \gg \theta_{b \bar{b}}$, the FSR correction for trimming becomes more like the plain jet where large angle corrections are strongly suppressed.

For the soft and collinear enhanced region $R_{\text {trim }} \ll \theta_{b \bar{b}}$, let us perform a more detailed calculation. First let us examine the loss in mass in more detail. One has:

$$
M_{H}^{2}-M_{j}^{2}=2\left(p_{b} \cdot k+p_{\bar{b}} \cdot k\right)=\omega\left(E_{b} \theta_{b k}^{2}+E_{\bar{b}} \theta_{\bar{b} k}^{2}\right) .
$$

Consider first that the gluon $k$ is emitted collinear to the $b$ quark with momentum fraction $x$, as shown in figure 5 . In this limit one can neglect the $E_{b} \theta_{b k}^{2}$ contribution above and set $\theta_{\bar{b} k}^{2} \approx \theta_{b \bar{b}}^{2}=\frac{\Delta}{z(1-z)}$. Requiring $M_{H}-M_{j}<\delta M$ and neglecting terms of order $\delta M^{2}$ relative to $M_{H} \delta M$ gives the mass window constraint $2 M_{H} \delta M>\omega p_{T} \Delta / z$. Next, defining $x$ as the energy fraction of the soft gluon w.r.t. the energy of the hard emitting prong i.e. 
$x=\omega /\left(z p_{T}\right)$, one can write the mass window constraint as the condition $x<2 \delta M / M_{H}$, where we used the fact that $\Delta=M_{H}^{2} / p_{T}^{2}$. Likewise the $f_{\text {cut }}$ cut expressed as a condition on $x$ just gives $f_{\text {cut }} / z>x$.

In the collinear limit, one can also simplify the angular integration in eq. (3.7) which then assumes a simple $d \theta^{2} / \theta^{2}$ form, resulting in a logarithmic contribution, $\ln \frac{\theta_{b \bar{b}}^{2}}{R_{\text {trim }}^{2}}$. It is also possible to perform the angular integration exactly i.e. beyond the collinear limit, to account for less singular soft large-angle contributions. More details of the derivation and results on the angular integration are provided in appendix A.

We can therefore express the soft-collinear contribution to the FSR corrections as:

$$
\varepsilon_{\mathrm{S}, \mathrm{FSR}}^{(1)}=2 C_{F} \frac{\alpha_{s}}{\pi} \int_{f_{\text {cut }}}^{1-f_{\text {cut }}} d z \ln \frac{\theta_{b \bar{b}}^{2}}{R_{\text {trim }}^{2}} \int \frac{d x}{x} \Theta\left(f_{\text {cut }} / z-x\right)\left[\Theta\left(\frac{2 \delta M}{M_{H}}-x\right)-1\right],
$$

where a factor of 2 has been inserted to account for an identical result from the region where $k$ is collinear to $\bar{b}$ rather than $b$ and virtual corrections have been introduced corresponding to the -1 term in square brackets.

Now let us write $\theta_{b \bar{b}}^{2}=\Delta /(z(1-z))$ and carry out the integration over $x$ which gives

$$
\varepsilon_{\mathrm{S}, \mathrm{FSR}}^{(1)}=-2 C_{F} \frac{\alpha_{s}}{\pi} \int_{f_{\text {cut }}}^{1-f_{\text {cut }}} d z\left(\ln \frac{\Delta}{R_{\text {trim }}^{2}}-\ln (z(1-z))\right) \ln \frac{f_{\text {cut }}}{z \epsilon} \Theta\left(f_{\text {cut }}-z \epsilon\right),
$$

where we introduced $\epsilon=\frac{2 \delta M}{M_{H}}$. The structure of the above result is, in essence, a double logarithmic form with a soft divergence in the limit $\delta M \rightarrow 0$ and an accompanying collinear divergence when $R_{\text {trim }} \rightarrow 0$. Let us take $R_{\text {trim }}^{2} \ll \Delta,{ }^{3}$ for simplicity ignore the accompanying $\ln (z(1-z))$ term, and integrate over $z$ to generate the following result:

$$
\varepsilon_{\mathrm{S}, \mathrm{FSR}}^{(1)}=-2 C_{F} \frac{\alpha_{s}}{\pi} \ln \frac{\Delta}{R_{\text {trim }}^{2}}\left[C_{1}\left(f_{\text {cut }}, \epsilon\right) \Theta\left(f_{\text {cut }}-\frac{\epsilon}{1+\epsilon}\right)+C_{2}\left(f_{\text {cut }}, \epsilon\right) \Theta\left(\frac{\epsilon}{1+\epsilon}-f_{\text {cut }}\right)\right],
$$

where

$$
\begin{aligned}
& C_{1}=\left(1-2 f_{\text {cut }}\right)+\left(1-2 f_{\text {cut }}\right) \ln \frac{f_{\text {cut }}}{\epsilon}+f_{\text {cut }} \ln f_{\text {cut }}-\left(1-f_{\text {cut }}\right) \ln \left(1-f_{\text {cut }}\right), \\
& C_{2}=\frac{f_{\text {cut }}}{\epsilon}-f_{\text {cut }}-f_{\text {cut }} \ln \frac{1}{\epsilon} .
\end{aligned}
$$

We note that for values of $\epsilon \ll f_{\text {cut }}$ the signal efficiency will be dominated by a $\ln \frac{f_{\text {cut }}}{\epsilon}$ term in the coefficient $C_{1}$. The presence of the $f_{\text {cut }}$ constraint however means that in practice such logarithms make only modest or negligible contributions for a wide range of values of $\epsilon$ or equivalently $\delta M$. This can be contrasted to the case of filtering, computed in ref. [34], which has an identical collinear divergence to that for trimming above, but where additionally the absence of an $f_{\text {cut }}$ condition leads to a much stronger $\ln \frac{1}{\epsilon}$ enhancement, which needs to be treated with resummation. It is also straightforward to include the effects of hard collinear radiation by considering the full $p_{g q}$ splitting function rather than just its

\footnotetext{
${ }^{3}$ In this limit, the leading order result eq. (3.2) is simply $1-2 f_{\text {cut }}$, i.e. the two subjets never form a single subjet and are always subject to the asymmetry condition $x>f_{\text {cut }}$.
} 
divergent $1 / x$ piece. In this region it is possible for the quark to fall below the $f_{\text {cut }}$ threshold and therefore to be removed. Such corrections do not come with soft enhancements and produce terms that vanish with either $f_{\text {cut }}$ or $\epsilon$, hence do not have a sizeable numerical effect that would require resummation. For this reason, we do not calculate these terms explicitly, continuing to work in the soft and collinear limit.

To make the above statements more explicit let us consider the situation at $p_{T}=$ $300 \mathrm{GeV}$ and choose $f_{\text {cut }}=0.1$. The zeroth order result for signal efficiency is then $\varepsilon_{\mathrm{S}}^{(0)}=$ $1-2 f_{\text {cut }}=0.8$. If one chooses a value of $R_{\text {trim }}=0.1$ then $\ln \left(\Delta / R_{\text {trim }}^{2}\right) \sim \ln 17$ and one may expect significant (collinear enhanced) radiative corrections. Choosing a larger $R_{\text {trim }}=0.3$ one can instead reduce $\ln \Delta / R_{\text {trim }}^{2}$ to $\sim \ln 2$, which is not enhanced and does not require resummation, implying a much more modest FSR contribution. However we should also examine the effect of this increased $R_{\text {trim }}$ on ISR and UE contributions.

For our choice of parameters it is evident from MC studies that we do not pay a significant price for the increased $R_{\text {trim }}$ value in terms of the ISR contribution. At the same value of $p_{T}$ the UE contribution for $R_{\text {trim }}=0.3$ is also small (see figure 6 ). If one moves to higher $p_{T}$, say $3 \mathrm{TeV}$, one should correspondingly lower $R_{\text {trim }}$. Here one has $\Delta=0.0017$ and choosing a value of $R_{\text {trim }} \sim 0.1$ would ensure a small FSR contribution as well as reduce the impact of ISR and the underlying event. This illustrates that by an appropriate choice of $R_{\text {trim }}$ one can negate large radiative losses due to FSR, without necessarily suffering from large ISR/UE effects. In general the optimal value of $R_{\text {trim }}$ will involve a trade-off between FSR radiative corrections and ISR/UE effects. We shall return to this point in section 7.

We should also examine the role of soft divergences that are formally important in the $\epsilon \rightarrow 0$ limit. Taking a value of $\delta M=2 \mathrm{GeV}$ leads to $\epsilon=0.032$. For our choice of $f_{\text {cut }}=0.1$, we have $\ln f_{\text {cut }} / \epsilon \sim \ln 3$, which is not a genuinely large logarithm. The overall coefficient of $-2 C_{F} \frac{\alpha_{s}}{\pi} \ln \Delta / R_{\text {trim }}^{2}$, which is given by the $C_{1}$ and $C_{2}$ terms in eq. (3.12), is for $\delta M=$ $2 \mathrm{GeV}$, approximately 1.58 and for $\delta M=10 \mathrm{GeV}$ approximately 0.34 , thus indicating that resummation of soft logarithms is not a necessity. Expressed as a percentage of the tree level result $1-2 f_{\text {cut }}$, the FSR corrections, as computed above, constitute a roughly two percent to ten percent effect for $\delta M$ ranging from $2 \mathrm{GeV}$ to $10 \mathrm{GeV}$, if one chooses $R_{\text {trim }}^{2} \simeq \Delta / 2{ }^{4}$

Hence, we find that even the leading soft-collinear enhanced contribution makes only modest contributions to the signal efficiency, at best comparable to pure order $\alpha_{s}$ corrections. The main implication of this finding is that full fixed-order calculations or combinations of fixed-order results with parton showers (see refs. $[58,59]$ for a review of the latter methods), would give a better description of the signal efficiency than pure soft showers. We further explore in appendix B, in somewhat more detail, the role of fixed-order calculations in a description of the signal efficiency.

Eq. (3.11) is intended to address the formal limit $R_{\text {trim }}^{2} \ll \Delta$. It indicates that choosing such small values of $R_{\text {trim }}$ is problematic due to degradation of the jet from FSR loss. In the opposite limit i.e. $R_{\text {trim }}^{2} \gg \Delta$ the FSR dependence will be similar to the plain jet mass i.e. one may expect FSR losses to be negligible. On the other hand eq. (3.5) for ISR

\footnotetext{
${ }^{4}$ As we shall see later, this constitutes a somewhat non-optimal choice for $R_{\text {trim }}$ and is made here for purely illustrative purposes, in order to estimate the size of soft but non-collinear enhanced effects.
} 

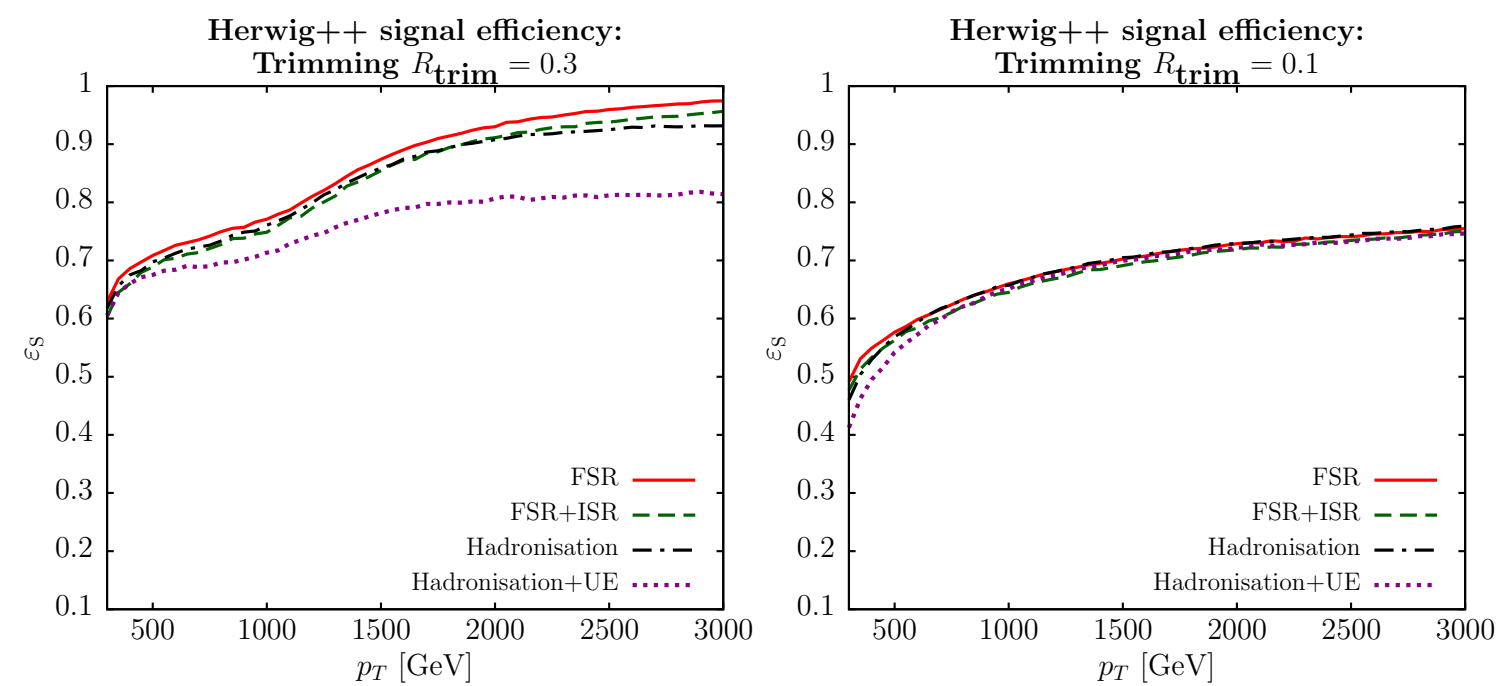

Figure 6. An MC study of the impact of hadronisation and underlying event (UE) on the signal efficiency using the the trimmed jet $\left(f_{\text {cut }}=0.1\right)$ as a function of the minimum jet transverse momentum for two different values of $R_{\text {trim }}$. One can see the impact of hadronisation and underlying event on the signal efficiency in the window $\delta M=16 \mathrm{GeV}$. Details of generation given in figure 2 .

corrections warns us that large choices of $R_{\text {trim }}$ may not be optimal due to increased ISR (and UE) contamination. One is thus led to think about the region $R_{\text {trim }}^{2} \sim \Delta$. This is reminiscent of the choice made in pruning for $R_{\text {prune }}^{2} \sim M_{j}^{2} / p_{T}^{2}$. In this limit the behaviour of FSR corrections for trimming is therefore expected to be similar to that for pruning for which a detailed calculation is carried out in section 4.1. We simply note here that in the region $R_{\text {trim }}^{2} \sim \Delta$ FSR corrections are relatively modest and can be thought of as pure order $\alpha_{s}$ corrections, rather than carrying significant logarithmic enhancements.

\subsection{Non-perturbative contributions}

Let us now study the impact of non-perturbative corrections to the signal efficiency using trimming on boosted Higgs jets.

In figure 6 we show the signal efficiency for a boosted Higgs signal jet after application of trimming with parameters $R_{\text {trim }}=0.3$ (left), $R_{\text {trim }}=0.1$ (right) with $f_{\text {cut }}=0.1$, as a function of the jet transverse momentum. One can see that hadronisation has little effect on the tagging rate of signal jets, due to the action of trimming on contributions which are soft and wide angle in the jet. UE has a larger impact on the signal efficiency due to soft contamination which is not checked for energy asymmetry. In other words inside the trimming radius the algorithm is inactive, and we automatically include all contamination coming from UE, which inside this region would contribute on average to a change in the jet mass squared varying as $R_{\text {trim }}^{4}$. The UE contribution could thus be substantially reduced by choosing a smaller $R_{\text {trim }}$. This is in particular required at higher $p_{T}$ as evident from figure 6 . Also, in contrast to the plain jet result in figure 2, one notes a significant reduction in sensitivity to non-perturbative effects when tagging signal jets using trimming. 


\section{Pruning and mMDT}

In this section we shall study pruning $[19,20]$ and the modified mass drop tagger $[3,31]$. We describe these methods together because unlike for the case of the QCD background studied in detail in ref. [31] where the taggers can exhibit substantial differences, for the signal one finds quite similar behaviour.

\subsection{Pruning}

Pruning uses the initial jet to calculate a pruning radius which is dependent on the mass of the jet and its transverse momentum $R_{\text {prune }}=R_{\text {fact }} \times \frac{2 M_{j}}{p_{T}}$ where $R_{\text {fact }}$ is a parameter of the tagger. It proceeds by reclustering the jet, at each step checking if both the angle between the two objects $i$ and $j$ is greater than the pruning radius $\Delta R_{i j}>R_{\text {prune }}$ and the splitting is $p_{T}$ asymmetric i.e. $\min \left(p_{T_{i}}, p_{T_{j}}\right)<z_{\text {cut }} \times p_{T_{(i+j)}}$. If these conditions are both true, pruning discards the softer of $i$ and $j$, else $i, j$ are combined as usual. This is repeated for each clustering step until all particles are either discarded or combined into the final pruned jet. For this study we use the default value $R_{\text {fact }}=\frac{1}{2}$ [19] and again use the C/A algorithm to both find and recluster the jets.

At zeroth order the two signal prongs are always at an angle larger than $R_{\text {prune }}$ and so the result is simply $1-2 z_{\text {cut }}$. For initial state radiation one can consider pruning to be similar to trimming with $R_{\text {trim }}$ replaced by $R_{\text {prune }}$. The pruning radius is given by

$$
R_{\text {prune }}^{2}=\frac{\left(p_{1}+p_{2}+k\right)^{2}}{p_{T}^{2}} \approx \Delta+\frac{2 p_{H} \cdot k}{p_{T}^{2}} \approx \Delta+x \theta^{2},
$$

where $\theta$ is the angle between the soft gluon and the Higgs direction (or equivalently, with neglect of recoil against soft ISR, the fat jet axis).

One then ends up comparing the gluon angle $\theta^{2}$ to $x \theta^{2}+\Delta$ and thus for sufficiently soft emissions i.e. in the limit $x \rightarrow 0$, responsible for logarithmic corrections, one can just replace $R_{\text {prune }}^{2}$ by $\Delta$. The situation is therefore identical to trimming but with $R_{\text {trim }}^{2}$ replaced by $\Delta$. Since we work in the limit $\Delta \ll 1$ we can neglect corrections varying as powers of $\Delta$, that replace the $R_{\text {trim }}$ dependence in eq. (3.5). The result should then be identical to trimming in that one should obtain a $\ln \frac{1}{z_{\text {cut }}}$, dependence with a transition to the plain mass behaviour visible for smaller $z_{\text {cut }}$ values as in figure 4 . We have verified that this is indeed the case with MC and that the efficiencies for pruning and trimming look essentially identical in terms of the response to ISR. An MC plot comparing ISR for all taggers is shown in the next section (figure 10), after we discuss the cases of mMDT, Y-pruning and Y-splitter.

Next we discuss briefly the situation with regard to FSR, in the context of pruning. Again one can employ the insight we gained in the previous section for the case of trimming. For the case of pruning there is no collinear enhancement since radiation that is lost is emitted at an angle (w.r.t. both hard prongs) larger than $R_{\text {prune }}^{2} \sim \Delta=z(1-z) \theta_{b \bar{b}}^{2}$ i.e. essentially of order $\theta_{b \bar{b}}^{2}$. Thus the angular integration produces a finite $\mathcal{O}(1)$ coefficient and we will thus obtain only a single logarithmic enhancement, that results from the loss of soft radiation at relative large angles i.e. those comparable to the $b \bar{b}$ dipole size. The 
corresponding loss in mass can be expressed as

$$
M_{H}^{2}-M_{j}^{2}=\left(p_{1}+p_{2}+k\right)^{2}-\left(p_{1}+p_{2}\right)^{2}=2 k \cdot\left(p_{1}+p_{2}\right) \approx \omega p_{T}\left(\theta^{2}+\Delta\right),
$$

where $\theta$ is the angle between the gluon and the jet direction. Noting that $\theta^{2}$ is at most of order $\Delta$ (contributions from the region where $\theta^{2} \gg \Delta$ are negligible due to angular ordering of soft radiation) one can replace $M_{H}^{2}-M_{j}^{2}$ by $\omega p_{T} \Delta$, ignoring any multiplicative factors of order one, that lead to only subleading logarithmic terms. The condition on gluon energy due to the mass window constraint is then $2 \frac{\delta M}{M_{H}}>\frac{\omega}{p_{T}}$. One also requires a constraint on the gluon energy such that it fails the $z_{\text {cut }}$ condition. ${ }^{5}$ In the soft limit and to leading logarithmic accuracy this condition is just that $\frac{\omega}{p_{T}}<z_{\text {cut }}$. Denoting $\frac{\omega}{p_{T}}$ by $x$, and accounting for virtual corrections as for the case of trimming, we have the expression for FSR corrections to pruning:

$$
\varepsilon_{\mathrm{S}, \mathrm{FSR}}^{(1)} \simeq-C_{F} \frac{\alpha_{s}}{\pi} \int_{z_{\mathrm{cut}}}^{1-z_{\mathrm{cut}}} d z \int \frac{d x}{x} \Theta\left(z_{\mathrm{cut}}-x\right) \Theta(x-\epsilon) \int \frac{(b \bar{b})}{(b k)(\bar{b} k)} \frac{d \Omega}{2 \pi} \Theta_{\mathrm{prune}}^{\mathrm{FSR}}
$$

where the condition $\Theta_{\text {prune }}^{\mathrm{FSR}}$ is simply the condition that the gluon is emitted outside an angle

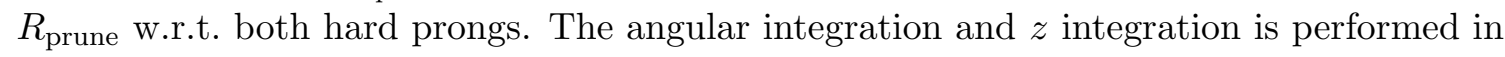
appendix A and carrying out also the integrals over $x$ we obtain the result:

$$
\varepsilon_{\mathrm{S}, \mathrm{FSR}}^{(1)}=-C_{F} \frac{\alpha_{s}}{\pi} \frac{2 \pi}{\sqrt{3}} \ln \frac{z_{\mathrm{cut}}}{\epsilon}, z_{\mathrm{cut}}>\epsilon .
$$

The above results suggest that logarithmic enhancements for pruning are in principle present for $\epsilon \ll z_{\text {cut }}$. However even with a choice of $\delta M$ as low as $2 \mathrm{GeV}$, with a choice of $z_{\text {cut }}=0.1$ we obtain a modest logarithm $\sim \ln 3$, implying that soft enhanced effects can be neglected. To therefore assess FSR corrections in more detail, as we found for trimming, it is necessary to go beyond the soft approximation and study hard corrections, which we explore further in appendix B. However it should be apparent that radiative corrections due to FSR corrections represent essentially order $\alpha_{s}$ corrections without significant log enhancements over a wide range of values of $\delta M, p_{T}$ and $z_{\text {cut }}$. We can exploit this stability against radiative corrections in optimising the tagger parameters, which we do in a subsequent section.

To obtain a complete picture of pruning we also need to account for non-perturbative corrections arising from hadronisation and UE corrections. We shall comment on MC results for these aspects together with the mMDT results below.

\subsection{Modified mass drop tagger}

Here we shall consider the modified mass drop tagger along similar lines. We start by recalling the definition first of the regular mass drop tagger: the mass drop tagger (MDT) [3] is intended for use with jets clustered using the $\mathrm{C} / \mathrm{A}$ algorithm. For each jet $j$ one applies the algorithm:

\footnotetext{
${ }^{5}$ Strictly, with the precise definition of the $z_{\text {cut }}$ condition we would have to consider a cut on $\omega$ normalised to the energy of the corresponding declustered prong i.e. $z p_{T}$ or $(1-z) p_{T}$ but at single-log accuracy one can just always take a cut on $\omega / p_{T}$.
} 
1. If jet $j$ contains subjets, split $j$ into two subjets $j_{1}$ and $j_{2}$ by undoing the last stage of clustering such that $m_{j_{1}}>m_{j_{2}}$.

2. If there is a significant mass drop $\mu \times m_{j}>m_{j_{1}}$ and the splitting is not too asymmetric $\min \left(p_{T, j_{1}}^{2}, p_{T, j_{2}}^{2}\right) \Delta R_{j_{1}, j_{2}}^{2} / m_{j}^{2}>y_{\text {cut }}$ deem $j$ to be a "tagged" jet and exit the loop.

3. Else relabel $j_{1}=j$ and repeat from step 1 .

The modified mass drop tagger corrected a flaw in the mass drop tagger so that in the event that the mass drop and asymmetry conditions are not satisfied one follows the more energetic (higher $p_{T}$ branch) rather than the heavier branch $j_{1}$ as advocated above. This is not only physically relevant (as one ensures that one identifies hard substructure rather than, for a small fraction of events, following soft massive jets) but also ameliorates significantly the perturbative structure for calculations related to QCD background jets, rendering for instance the QCD jet mass distribution purely single logarithmic and free from non-global logarithms.

One other observation that was made in ref. [31] concerned the role of the mass drop parameter $\mu$ itself. There it was noted that the mass drop condition had a negligible impact on the result obtained for the jet mass distribution for QCD background jets and hence that it was possible to entirely ignore the mass drop requirement. For the current paper we shall consider this variant of the mMDT, where we do not impose the mass-drop condition but just the asymmetry requirement via a $y_{\text {cut }}$ cut-off.

At zeroth order we obtain a signal efficiency $\varepsilon_{\mathrm{S}}^{(0)}=1-2 y_{\text {cut }}$ coming from the asymmetry cut, which is the same result as for pruning. As far as the response to ISR is concerned, one can straightforwardly see that the general behaviour will be similar to the taggers we have considered before. Consider a fat jet consisting of a $b \bar{b}$ pair and an ISR gluon. If the gluon makes an angle less than $\theta_{b \bar{b}}$ with either of the hard prongs of the fat jet then on declustering, we will break the jet into a massless prong and a prong with a small mass consisting of a quark and the soft gluon. In the soft limit the asymmetry condition will pass if the hard prongs are sufficiently energetic, i.e. exactly as at zeroth order, and the soft ISR will contaminate the jet. Such corrections will vanish with $M_{H} / p_{T}$ just like for the case of pruning and hence we can ignore them here, at high $p_{T}$. For relatively large angle ISR, the soft gluon emerges first on declustering the jet. If it fails the asymmetry condition it is removed and its effects cancel against virtual corrections. If it passes the asymmetry cut one obtains, in the small $\Delta$ limit, essentially a logarithm in $y_{\text {cut }}$ as for pruning and trimming. One should note that, if one uses the asymmetry condition, with the $y_{\text {cut }}$ measure exactly as defined above, the condition for the gluon to pass the asymmetry can be expressed as $x^{2} \theta^{2} /\left(\Delta+x \theta^{2}\right)>y_{\text {cut }}$, where $x=\omega / p_{T}$. Combining this with the condition $\theta^{2}<R^{2}$, on the angular integration, we get the constraint:

$$
x>\frac{y_{\text {cut }}}{2}\left(1+\sqrt{1+\frac{4 \Delta}{y_{\text {cut }} R^{2}}}\right),
$$

which for $y_{\text {cut }} \gg \Delta / R^{2}$ reduces to the same constraint as for the case of pruning and trimming i.e. $x>y_{\text {cut }}$. The main effect of this slightly different relationship between the gluon 
energy and $y_{\text {cut }}$ manifests itself, only for rather small $y_{\text {cut }}$ values and at low $p_{T}$, in terms of a change in the transition point to the plain jet mass like behaviour. The position of the transition can be computed as before by setting the r.h.s. of eq. (4.5) equal to $\frac{2 M_{H} \delta M}{p^{2}}$ recalling that we take the fat jet radius $R=1$. Let us consider $f_{\text {cut }}=0.005$ and $\delta M=16 \mathrm{GeV}$, for which one obtains a transition point with trimming at $p_{T} \sim 900 \mathrm{GeV}$, below which one sees a plain jet like behaviour. For the same value of $y_{\text {cut }}$ the corresponding transition point for mMDT occurs at roughly $p_{T} \sim 400 \mathrm{GeV}$, i.e. is absent over the range of $p_{T}$ considered here. In any case very small values of $y_{\text {cut }}$ would mean that large logarithms in $y_{\text {cut }}$ become important and hence should in general be avoided. For reasonable values of $y_{\text {cut }} \sim 0.1$, mMDT behaves essentially identical to pruning and trimming. We have verified all of the above points with MC studies and shall provide a plot comparing the response of taggers to ISR in the next section, after discussing Y-pruning and Y-splitter (see figure 10). Lastly we shall mention that for FSR corrections in the soft approximation, we do not observe any significant differences between mMDT and pruning. To understand this it is enough to realise that a soft FSR gluon emitted at an angle smaller than $\theta_{b \bar{b}}$ w.r.t. either the $b$ or $\bar{b}$ direction is not examined for the asymmetry condition and hence does not contribute to a loss in jet mass, implying also the absence of any collinear enhancements. Emissions at an angle larger than $\theta_{b \bar{b}}^{2}=\frac{\Delta}{z(1-z)}$ contribute to a loss in mass and give a soft single logarithmic contribution identical to that for pruning. The result obtained is identical to eq. (4.4) with the coefficient $2 \pi / \sqrt{3} \approx 3.63$ replaced by a coefficient that we have determined numerically. For $p_{T}=3 \mathrm{TeV}$ the coefficient we obtain is $\approx 0.646 .{ }^{6}$ The key point however is that no large logarithmic corrections arise due to soft FSR emissions, owing to the presence of the $y_{\text {cut }}$ cut-off. Once again it would therefore be of interest to study the role of genuinely hard radiative corrections beyond the eikonal approximation, a study we carry out in appendix B.

\subsection{Non-perturbative effects and MC results}

We have analysed the effects of ISR and FSR for both pruning and mMDT and concluded that the taggers have an essentially similar behaviour for the case of signal jets. Of course our studies have focussed thus far on the perturbative component and hence it is prudent to examine non-perturbative effects before reaching any firm conclusions.

We show in figure 7 the MC results for pruning and mMDT. One observes that the signal efficiency has only a weak dependence on $p_{T}$ and that relative to the lowest order expectation of $\varepsilon_{\mathrm{S}}^{(0)}=0.8$, at high $p_{T}$ one sees a roughly 10 percent difference for the full parton level result with radiative corrections. One also sees a remarkable similarity between the two taggers over the entire $p_{T}$ range as far as parton level results and those including hadronisation are concerned. The UE contamination is however more clearly visible in the mMDT case towards lower $p_{T}$ values which owes to the larger effective radius $\theta_{b \bar{b}}=\frac{M_{H}}{p_{T} \sqrt{z(1-z)}}$ as compared to $R_{\text {prune }} \approx M_{H} / p_{T}$ for pruning as well as differences in the definitions of the asymmetry parameters $y_{\text {cut }}$ vs $z_{\text {cut }}{ }^{7}$

\footnotetext{
${ }^{6}$ This is the same result, $J(1)$, found by Rubin in ref. [34] for the coefficient of the FSR soft logarithm for filtering when $R_{\text {filter }}=\theta_{b \bar{b}}$.

${ }^{7}$ It is of course possible to use mMDT with a $z_{\text {cut }}$ constraint defined as for pruning instead of $y_{\text {cut }}$, as
} 

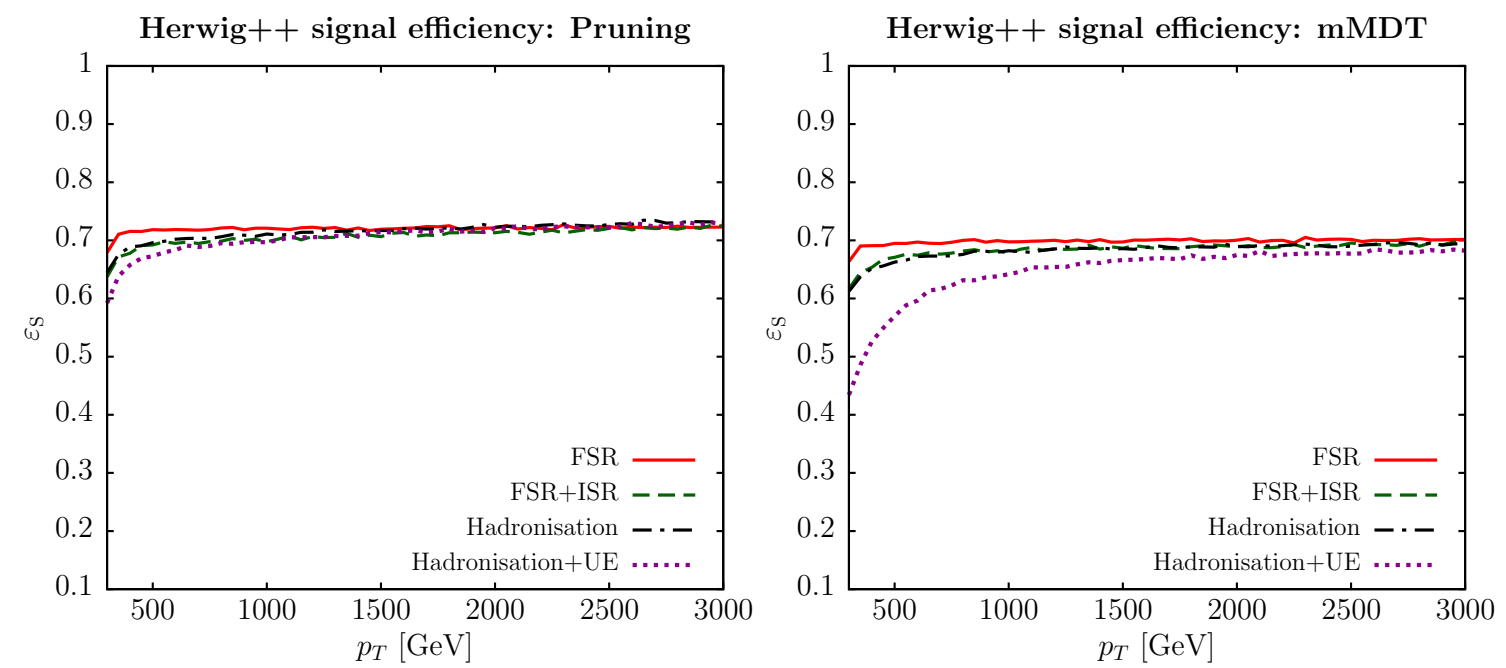

Figure 7. An MC study of the impact of hadronisation and underlying event (UE) on the signal efficiency for pruning (left) and mMDT (right) $\left(z_{\text {cut }}, y_{\text {cut }}=0.1\right)$ as a function of jet transverse momentum with $\delta M=16 \mathrm{GeV}$. Details of generation are given in figure 2 .

At lower $p_{T}$ therefore it has been standard practice to use the mass drop tagger in conjunction with filtering as suggested in the original reference ref. [3]. One should also bear in mind the results of ref. [31] where for QCD background jets much more pronounced nonperturbative effects were observed for pruning than for $\mathrm{mMDT}$, and in the final analysis one expects the impact on the background to dictate the ultimate performance of the taggers, rather than the comparatively small corrections one sees here for the signal, over most of the $p_{T}$ range studied.

A final point to make about figure 7 is about the contrast between the FSR corrections observed for mMDT and pruning to those seen in figure 6 for trimming. To make the comparison we note the fact that for figure 6 we have chosen $f_{\text {cut }}=0.1$ and consider $R_{\text {trim }}=0.1$. Then the zeroth order result for trimming is simply $1-2 f_{\text {cut }}$ as for mMDT and pruning, within the $p_{T}$ range we are studying. It is evident from figures 6 and 7 that while the FSR results for mMDT and pruning show hardly any dependence on $p_{T}$ over the range studied, the corresponding results for trimming show a more pronounced $p_{T}$ dependence. This feature already emerges from our simplified fixed-coupling analytics where the FSR corrections for trimming depend on $p_{T}$ via a dependence on $\ln \Delta / R_{\text {trim }}^{2}$ while for pruning and mMDT we have shown that the FSR corrections are $p_{T}$ independent (see e.g. eq. (4.4) for pruning). We shall return to this point in section 7.

\section{$5 \quad$ Y-pruning and Y-splitter}

In this section we shall study the Y-pruning modification of pruning suggested recently [31], along with the older Y-splitter method [2]. We shall study these two methods

was studied in ref. [31]. This choice would further enhance the similarity we observe for signal jets and is the default in the current public implementation of mMDT in FastJet [50]. 
together because they have a remarkably similar action on QCD background jets, and are particularly effective in cutting out QCD background in the vicinity of signal peaks, for boosted Higgs and electroweak gauge bosons, which makes them potentially valuable tools. They however differ very significantly from each other and from other taggers in their response to initial state radiation (and even more significantly to UE), for reasons we highlight below, and which were also mentioned in the case of Y-pruning, in ref. [31]. In the next subsection we shall use the insight we gain in the present section to suggest improvements to $\mathrm{Y}$-splitter in particular.

\section{$5.1 \quad$ Y-pruning}

We begin by examining the case of Y-pruning. Let us recall that this is a modification of pruning where one requires that at least one clustering is explicitly checked for and passes the pruning criteria else one discards the jet. In this way one removes spurious configurations where all emissions that are left after pruning is applied, are within an angular distance $R_{\text {prune }}$ of one another and hence never get examined for an asymmetry condition, resulting in the tagging of structures with only a single hard prong.

A known issue with Y-pruning for the case of signal jets, already discovered in ref. [31], concerns its response to soft wide-angle emissions from ISR or UE. Here one can have a situation where a soft ISR emission contributes to setting the pruning radius but is itself removed by pruning. If the pruning radius set by the ISR emission is larger than $\theta_{b \bar{b}}$ then one would discard the resulting jet as it does not satisfy Y-pruning, causing a loss of signal. In the same kinematic region virtual corrections would lead to a jet accepted by Y-pruning (assuming the hard prongs arising from Higgs decay satisfy the $z_{\text {cut }}$ criterion), and hence contribute to the signal tagging efficiency, as we shall demonstrate below.

We first consider that for an ISR emission with energy, or equivalently $k_{t}$, fraction $x \ll 1$ which makes an angle $\theta$ with the fat jet axis (again we neglect recoil against soft ISR), we have that $R_{\text {prune }}^{2}=M_{j}^{2} / p_{T}^{2} \approx \Delta+x \theta^{2}$. We wish to compute the virtual contribution in the region where the real ISR is removed i.e. in the kinematic region where $\theta^{2}>R_{\text {prune }}^{2}$ and where $x<z_{\text {cut }}$. Moreover we require that $R_{\text {prune }}^{2}>\theta_{b \bar{b}}^{2}$ so that the hard prongs are inside the pruning radius.

Thus we have, for the contribution of uncancelled virtual gluons, the equation

$$
\Delta \varepsilon_{\mathrm{S}}=-C_{F} \frac{\alpha_{s}}{\pi} \int_{z_{\text {cut }}}^{1-z_{\text {cut }}} d z \int \frac{d x}{x} d \theta^{2} \Theta\left(\theta^{2}-\Delta\right) \Theta\left(\theta^{2}-\frac{\Delta}{x} f(z)\right) \Theta\left(R^{2}-\theta^{2}\right) \Theta\left(z_{\text {cut }}-x\right),
$$

where we have denoted the extra contribution for Y-pruning, relative to pruning, by $\Delta \varepsilon_{\mathrm{S}}$, have defined the function $f(z)=\frac{1}{z(1-z)}-1$ and where the overall minus sign indicates that we are considering the virtual contribution. The first step function in the above equation comes from the requirement that we are considering the region where the ISR emission lies at a larger angle than $R_{\text {prune }}$ relative to the jet axis (with $x \ll 1$ ) while the second step function expresses the constraint that the $b \bar{b}$ opening angle is less than $R_{\text {prune }}$, the pruning radius. Lastly we have conditions corresponding to the ISR radiation being in the fat jet $R^{2}>\theta^{2}$ and the energy condition $x<z_{\text {cut }}$ corresponding to removal of the 

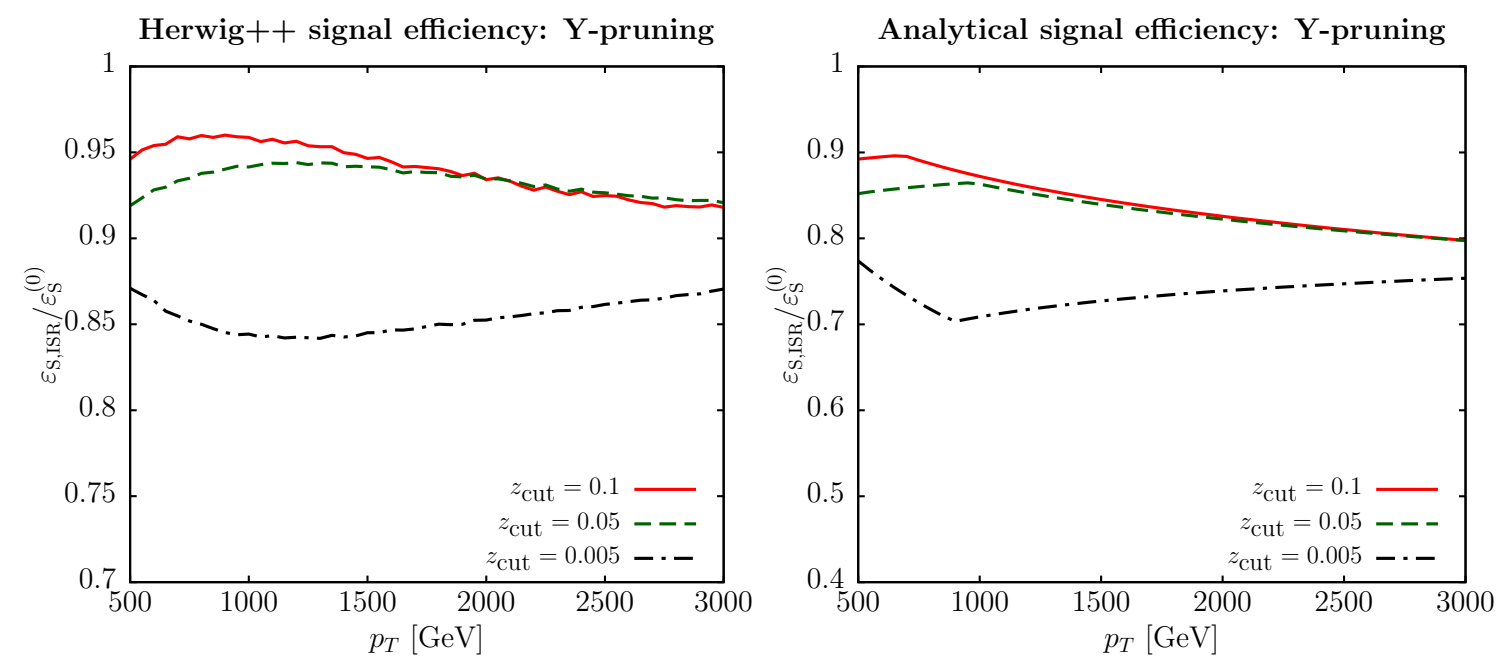

Figure 8. Comparison of MC (left) and analytic (right) Y-pruning signal tagging efficiencies for a range of $z_{\text {cut }}$ values as a function of a generator level cut on jet transverse momentum. This result has been generated using Herwig ++2.7 .0 [44] at parton level with ISR only for $H \rightarrow b \bar{b}$ jets, setting $M_{H}=125 \mathrm{GeV}$ and $\delta M=16 \mathrm{GeV}$. We have divided out the contribution due to the born configuration in both panels for clarity.

corresponding real radiation. We can straightforwardly carry out the integrals over $\theta^{2}, x$ and $z$. In particular the integration over $x$ produces the logarithmically enhanced term we seek, where one obtains a logarithm in the ratio of $z_{\text {cut }}$ to $\Delta$. One may expect that this logarithm becomes large and hence should have a visible effect at high $p_{T}$, for values of $z_{\text {cut }}$ that are not too small. The final result, discarding all other terms that are less singular in the high $p_{T}$ limit (e.g. those that vanish with $\Delta$ ), is

$$
\begin{aligned}
\Delta \varepsilon_{\mathrm{S}} \approx-C_{F} \frac{\alpha_{s}}{\pi} R^{2} \ln \frac{z_{\text {cut }} R^{2}}{\Delta} \Theta(\beta-3) & {\left[\sqrt{1-\frac{4}{1+\beta}} \Theta\left(\frac{1}{1+\beta}-z_{\text {cut }}\left(1-z_{\text {cut }}\right)\right)\right.} \\
& \left.+\left(1-2 z_{\text {cut }}\right) \Theta\left(z_{\text {cut }}\left(1-z_{\text {cut }}\right)-\frac{1}{1+\beta}\right)\right],
\end{aligned}
$$

where we defined $\beta=\frac{z_{\text {cut }} R^{2}}{\Delta}$.

At high $p_{T}$ therefore, $\Delta \varepsilon_{S}$ potentially dominates the normal logarithmic dependence of pruning on $z_{\text {cut }}$. We can therefore distinguish Y-pruning from other taggers by looking at the transverse momentum dependence of the signal response to ISR in the high $p_{T}$ limit.

In figure 8 we compare the sum of the analytic result from pruning and the additional contribution described in eq. (5.2) to MC with ISR for a range of $z_{\text {cut }}$ values. One immediately notices, in both analytical and $\mathrm{MC}$ plots, that the $p_{T}$ dependence of Y-pruning is significantly different from that of pruning for the commonly used value $z_{\text {cut }}=0.1$, (see figure 10). In agreement with our expectations the signal efficiency as given by MC in figure 8 first increases with $p_{T}$ as for the case of pruning and then decreases beyond a certain point which we expect to be the onset of the logarithmic behaviour we have computed above. Our calculation indicates that the onset of the logarithm in $z_{\text {cut }} / \Delta$ is for $\beta>3$ which for 


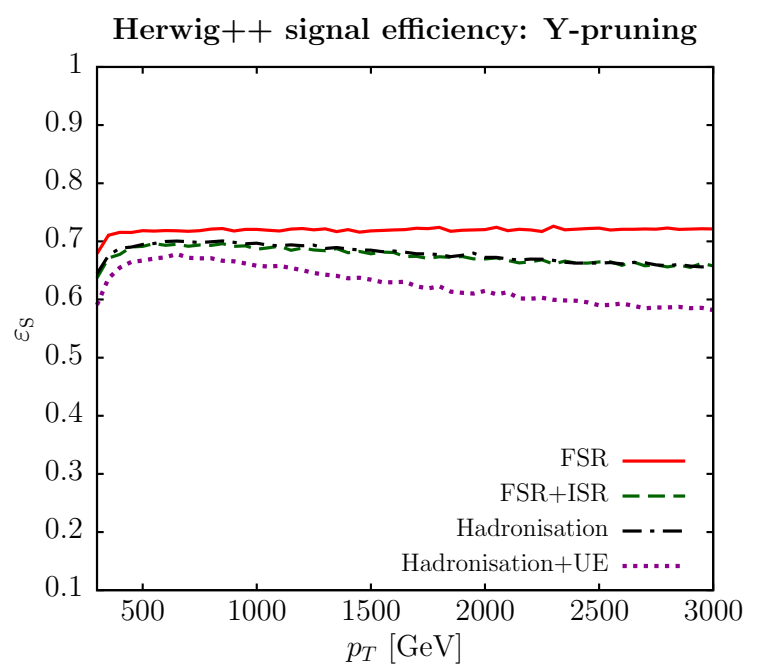

Figure 9. An MC study of the impact of hadronisation and underlying event (UE) on the signal efficiency using the the Y-pruned jet $\left(z_{\text {cut }}=0.1\right)$ as a function of the minimum jet transverse momentum. One can see negligible impact coming from hadronisation and but some degradation coming from underlying event contamination on the signal efficiency in the window $\delta M=16 \mathrm{GeV}$. Details of generation given in figure 2 .

$z_{\text {cut }}=0.1$ corresponds to approximately $680 \mathrm{GeV}$ and for $z_{\text {cut }}=0.05$ to approximately $970 \mathrm{GeV}$. This is consistent with what is seen in MC though of course the transitions are not as sharp as in the analytical result.

As far as final state radiation is concerned there is no significant difference between Y-pruning and pruning. The soft large-angle contributions that are responsible for the loss of signal we saw for ISR are strongly suppressed for the case of FSR, due to the colour singlet nature of the parent Higgs particle and angular ordering. We conclude by showing in figure 9 an MC plot for Y-pruning at both parton level (including both ISR and FSR) and with non-perturbative corrections. As expected there is some significant loss of signal due to UE contributions for precisely the same reasons as for the case of ISR and as also observed in ref. [31]. In spite of this deficiency it was also shown in ref. [31] that due to its strong suppression of background jets Y-pruning produced a signal significance that was at least comparable and at high $p_{T}$ exceeded that from the other taggers studied (mMDT, pruning and trimming), especially for gluon jet backgrounds. In the next section we shall study an older method, Y-splitter, that has a similar action to Y-pruning for background jets, which makes its action on signal worth exploring further.

\section{$5.2 \quad$ Y-splitter}

The Y-splitter technique was first introduced in ref. [2] in the context of $\mathrm{W}$ boson tagging. The main observation was that the $k_{t}$ distance measure (as employed in the $k_{t}$ algorithm [60]) between the two partonic prongs of a $W$ decay tended to be close to the $W$ mass, which is a consequence of a typically symmetric energy sharing between the two prongs, in contrast to the case of QCD background where the energy sharing is typically asymmetric. To exploit this fact one takes a fat jet constructed with the $k_{t}$ algorithm and 
undoes the last step of the clustering. This produces two prongs and we let them have energy fractions $z$ and $1-z$. The $k_{t}$ distance $d_{i j}$ is given, at small opening angles, by the square of the transverse momentum of the softer prong w.r.t. the direction of the harder prong which gives:

$$
d_{i j}=\min (z, 1-z)^{2} p_{T}^{2} \theta_{i j}^{2}
$$

One can either cut directly on this distance by requiring it to be of order $M_{W}^{2}\left(M_{H}^{2}\right.$ in our case) or cut on the ratio of $d_{i j}$ to the jet invariant mass squared $M_{j}^{2}=p_{T}^{2} z(1-z) \theta_{i j}^{2}$ (see e.g. ref. [29, 61]). In the present case we shall choose the latter option and hence demand that

$$
\frac{d_{i j}}{M_{j}^{2}}=\frac{\min (z, 1-z)}{\max (z, 1-z)}>y_{\mathrm{cut}} .
$$

If this condition is satisfied then one tags the jet else one discards it. Taking for instance $z<1-z$ the cut amounts to requiring that $z>y_{\text {cut }} /\left(1+y_{\text {cut }}\right)=y_{\text {cut }}-\mathcal{O}\left(y_{\text {cut }}^{2}\right)$. Likewise for $z>1-z$ one obtains $z<1-y_{\text {cut }}+\mathcal{O}\left(y_{\text {cut }}^{2}\right)$.

The Y-splitter method has not been as widely used in recent times as some of the other methods we have studied here, though one relatively recent application has been for the purposes of top tagging [15]. Also, a detailed comparison of Y-splitter with N-subjettiness was carried out in ref. [62].

Let us first consider the action of Y-splitter on QCD background. If one considers a quark jet with an additional soft-collinear gluon emission, then the $y_{\text {cut }}$ condition is active on the gluon energy, which means it regulates the soft divergence associated to gluon emission. The usual double logarithmic structure of the QCD jet mass gives way to a single logarithmic answer precisely as for the mMDT, pruning and Y-pruning methods [31]:

$$
\frac{\rho}{\sigma} \frac{d \sigma}{d \rho}^{(\text {Y-splitter,LO })} \simeq C_{F} \frac{\alpha_{s}}{\pi}\left(\ln \frac{1}{y_{\text {cut }}}-\frac{3}{4}\right), \rho<y_{\text {cut }},
$$

with $\rho=\frac{M_{j}^{2}}{p_{T}^{2} R^{2}}$ and where we neglected terms varying as powers of $y_{\text {cut }}$. For $\rho>y_{\text {cut }}$ one obtains a transition to the normal jet mass result.

At all orders the result for Y-splitter can be derived using methods similar to those in ref. [31]. Since the derivation of this result takes us away from our current focus on signals, we shall not provide it here, but shall do so in a forthcoming paper [63]. The basic fixed coupling result, for small $\rho$ can be expressed in the form:

$$
\frac{\rho}{\sigma}{\frac{d \sigma^{(Y-s p l i t t e r)}}{d \rho}}^{\left(C_{F}\right.} \frac{\alpha_{s}}{\pi}\left(\ln \frac{1}{y_{\text {cut }}}-\frac{3}{4}\right) \exp \left[-\frac{C_{F} \alpha_{s}}{2 \pi} \ln ^{2} \frac{1}{\rho}\right]
$$

which represents a Sudakov suppression of the leading order result. The form of this result is identical to that derived for Y-pruning in the region $\rho<z_{\text {cut }}^{2}$ and when $\alpha_{s} \ln \frac{1}{z_{\text {cut }}} \ln \frac{1}{\rho} \ll 1$ (see eq. (5.10b) of ref. [31]), though subleading logarithmic terms will differ. One can verify this similarity of Y-splitter to Y-pruning, for the case of QCD jets, by examining the results produced by MC and we shall do so in the next subsection.

Next we shall study the response of Y-splitter to signal jets, for our case of Higgs decays. At zeroth order the result is similar to that for mMDT and pruning and with 
neglect of order $y_{\text {cut }}^{2}$ terms one simply gets $\varepsilon_{\mathrm{S}}^{(0)}=1-2 y_{\text {cut }}$, which is as usual a consequence of the uniform $z$ distribution and the asymmetry cuts on $z$.

Beyond zeroth order however one should expect very significant differences between Y-splitter and the other taggers. These come essentially from the response to ISR (and UE and as one can expect also from pile-up). In order to understand the ISR response let us consider our usual configuration of a $b \bar{b}$ pair and a large-angle soft ISR gluon with $\theta \sim 1 \gg \theta_{b \bar{b}}$. There are two configurations of interest. Firstly when the distance $d_{i j}$ between the ISR gluon and both the hard prongs is larger than that of the $b \bar{b}$ quark pair one examines $k_{t}^{2} / M_{j}^{2}$ where $k_{t}$ is the transverse momentum of the gluon w.r.t. the jet axis, and require that this be greater than $y_{\text {cut }}$, for the jet to be retained. Also we require that the mass window constraint is satisfied as for the plain jet mass.

On the other hand when the $d_{i j}$ between the gluon and the $b \bar{b}$ pair is smaller than that between the $b$ and $\bar{b}$ prongs, the gluon is simply clustered into the jet and one just imposes the $y_{\text {cut }}$ condition on the two hard prongs as at zeroth order. The gluon thus contaminates the jet and one has to impose the mass window constraint again as for the plain jet mass.

In fact one can argue that the first configuration where the gluon has a larger $k_{t}$ distance, along with the fact that it should not be too energetic so as to comply with the mass window constraint, is limited to a small corner of phase space that vanishes with $\delta M / p_{T}$, where $\delta M$, as before, is the size of the window.

To see this most straightforwardly one notes that the gluon has a $k_{t}$ distance from the $b \bar{b}$ pair (or equivalently in our soft large-angle approximation from either the $b$ or $\bar{b}$ ) which is given essentially by $d_{i j}=x^{2} p_{T}^{2} \theta^{2} \simeq x^{2} p_{T}^{2}$ where $x=\omega / p_{T}$. On the other hand we have the $k_{t}$ distance between the $b$ and $\bar{b}$ is $\min (z, 1-z)^{2} p_{T}^{2} \theta_{b \bar{b}}^{2}$, and let us for convenience suppose that $z<1 / 2$. One requires therefore that $x^{2} p_{T}^{2}>z^{2} p_{T}^{2} \theta_{b \bar{b}}^{2}$, while the mass window condition again for $\theta^{2} \sim 1 \gg \Delta$ gives $x<\frac{2 M_{H} \delta M}{p_{T}^{2}}$. These conditions are only simultaneously satisfied if $z /(1-z)<\left(2 \delta M / p_{T}\right)^{2}$, which corresponds to a negligibly small region of phase space and given the uniform distribution in $z$, can be ignored. Hence we are left with the situation that, modulo small corrections, the ISR gluon contaminates the jet and gives a result that is essentially like the plain jet mass. This implies considerable degradation in mass due to ISR and further due to UE and, of course in the final analysis, pile-up.

Let us compare Y-splitter with the other taggers and the plain jet mass using MC. In figure 10 we show the response of all taggers studied thus far to ISR, as a function of jet $p_{T}$. One can immediately see that Y-splitter and the plain jet mass are essentially identical. One also notes that mMDT, trimming and pruning have a very similar behaviour to one another as we expected from our analytical estimates while Y-pruning suffers at high $p_{T}$ as already observed, while still remaining far better than Y-splitter.

As far as FSR is concerned, one does not expect any significant issues with Y-splitter. In contrast to ISR a soft FSR gluon will nearly always be clustered with the hard emitting partons, as a consequence of its softness and angular ordering, and so end up as part of the fat jet, thus not contributing to a loss in mass. Its effects will cancel against soft virtual corrections leaving us to study genuinely hard non-collinear configurations which ought to have a relatively modest impact at the level of pure order $\alpha_{s}$ corrections. 


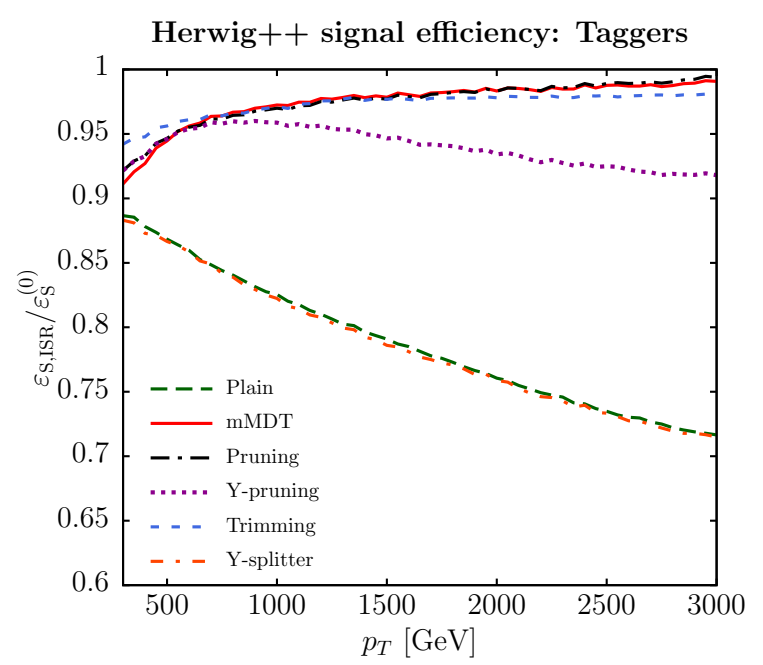

Figure 10. A MC study of the impact of ISR on the signal efficiency for various taggers ( $\left.y_{\text {cut }}, z_{\text {cut }}, f_{\text {cut }}=0.1, R_{\text {trim }}=0.3\right)$ as a function of the minimum jet transverse momentum. One can note the similarity of mMDT, pruning and trimming while Y-splitter and Y-pruning are different, with Y-splitter in particular virtually indistinguishable from the plain jet mass. Details of generation given in figure 1.

As far as NP effects are concerned we have also carried out MC studies for Y-splitter with hadronisation and UE. The findings here are that the effects are comparable in size to the plain jet mass.

Thus in the final analysis it appears that Y-splitter may not be as useful as the other methods studied here and in particular Y-pruning, even though it shares a very similar suppression of the QCD background. While Y-splitter appears effective at identifying hard substructure and removing background, it is not effective in grooming away soft contamination, as is inbuilt to varying extents in the other methods we have studied. This suggests using Y-splitter along with another method more effective in grooming may alleviate some of the issues we see with the signal. Therefore in the next subsection we shall consider its combination with trimming, which we find has some noteworthy features and produces interesting results.

\section{$6 \quad$ Y-splitter with trimming}

Here we shall study the combination of Y-splitter with trimming, in view of the lack of any effective grooming element in Y-splitter, as mentioned above. We do not have to necessarily choose trimming in this respect and it is possible to study a combination of Y-splitter with other methods such as mMDT and the recently introduced soft drop method [33]. Indeed it has been known for some time that combinations of substructure tools can often produce better results than the individual tools themselves [64] and thus one may hope to improve the performance of Y-splitter using a suitable complementary tool.

We first study the impact of applying trimming on signal jets that are tagged by Ysplitter. For the combination of Y-splitter with trimming we choose $f_{\text {cut }}=y_{\text {cut }}=0.1$ and $R_{\text {trim }}=0.3$. 


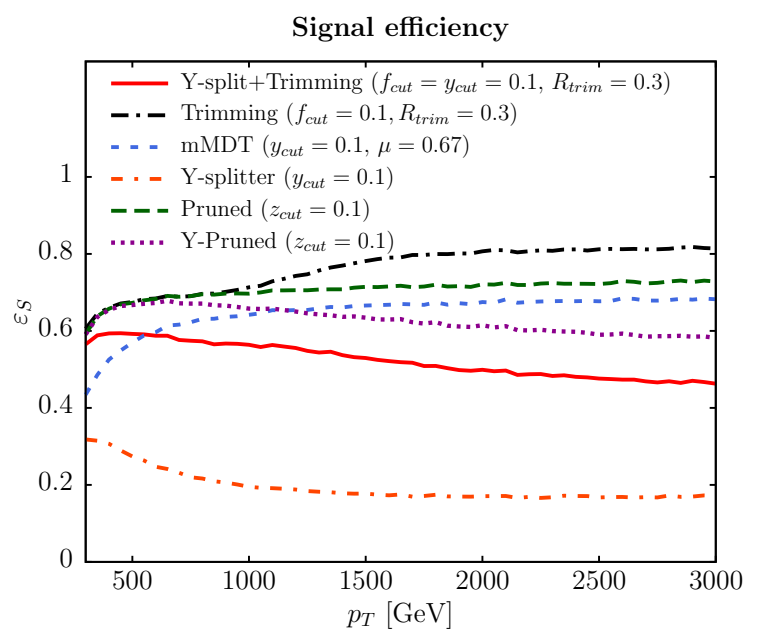

Figure 11. Signal efficiency for tagging hadronic $H$ jets using Herwig ++2.7 .0 [44] with underlying event and hadronisation as a function of a generator level cut $p_{T}$ on transverse jet momentum.

The MC analysis is shown in figure 11 which demonstrates that the use of trimming substantially ameliorates the loss of signal we saw with Y-splitter. It is evident from the same figure that while $\mathrm{Y}$-splitter with trimming still does not reach the signal efficiency of some other methods, the difference is much less pronounced than before. In fact one observes that the signal efficiency for Y-splitter with trimming bears a qualitative similarity to Ypruning. The reason for this is that the use of trimming turns the plain mass like behaviour of Y-splitter into the behaviour for trimming, except for configurations that have been rejected by Y-splitter, on which subsequent trimming does not act. This corresponds to the Y-splitter rejection region whereby an ISR gluon has the largest $k_{t}$ distance but fails the $y_{\text {cut }}$ requirement. This kinematic configuration is reminiscent of that which resulted in the extra $\Delta \varepsilon_{\mathrm{S}}$ term for Y-pruning. In the present case uncancelled virtual corrections integrated over the Y-splitter rejection region produce a term $\sim-C_{F} \frac{\alpha_{s}}{\pi} R^{2} \ln \frac{y_{\text {cut }}}{\sqrt{\Delta}}$, which corrects the simple trimming result. However the overall efficiency remains considerably higher than the Y-splitter or plain mass result, due to elimination of the plain jet mass like logarithm.

Next one should study also the impact of using trimming in conjunction with Ysplitter on the QCD background. In this article, given our focus on signal jets, we shall not attempt to provide a detailed analytical study of the background case, which together with the derivation of the basic Y-splitter formula eq. (5.6) we shall carry out in a forthcoming article [63]. Here we shall confine ourselves to MC studies and the result of these is shown in figure 12. Once again we study the action of trimming on jets that have been tagged by Y-splitter where we choose $f_{\text {cut }}=y_{\text {cut }}=0.1$ for both Y-splitter and trimming and $R_{\text {trim }}=0.3$. In this plot, we remind the reader that $\rho=\frac{M_{j}^{2}}{p_{T}^{2} R^{2}}$ i.e. the normalised jet mass as defined and used in ref. [31].

One notes from figure 12 that Y-pruning, Y-splitter and Y-splitter+trimming all have a fairly similar action on background jets and provide, for our choice of parameters, a significant suppression of background around the signal mass-peak $\sim 100 \mathrm{GeV}$. These results are for quark backgrounds but similar results are obtained for gluon jets. It is noteworthy 


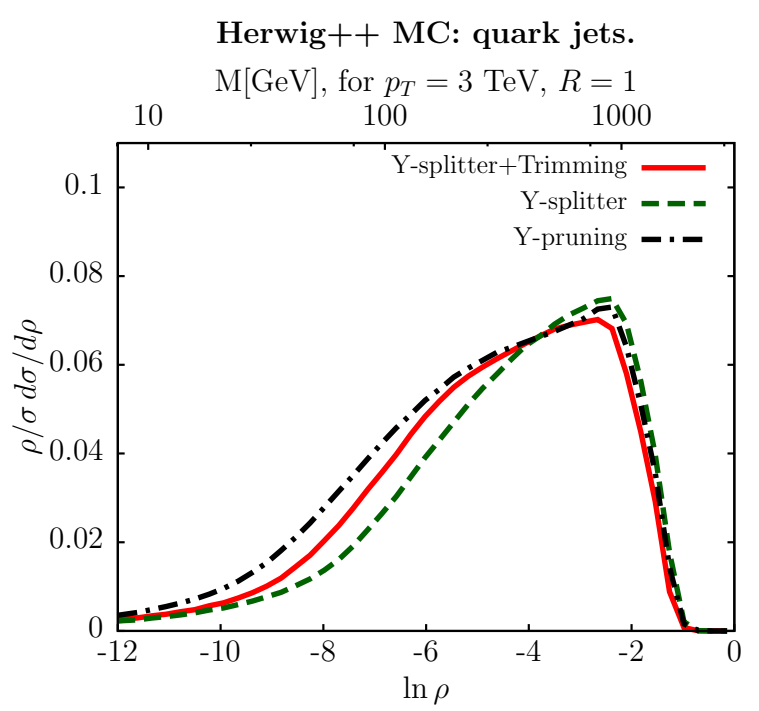

Figure 12. MC results for the differential jet mass distribution using Y-splitter with trimming $\left(y_{\text {cut }}=0.1, R_{\text {trim }}=0.3\right)$, Y-splitter $\left(y_{\text {cut }}=0.1\right)$ and Y-pruning $\left(z_{\text {cut }}=0.1\right)$. This result has been generated using Herwig ++2.7 .0 [44] at parton level. A minimum $p_{T}$ cut on generation of the hard process $q q \rightarrow q q$ was made at $3 \mathrm{TeV}$ for $14 \mathrm{TeV}$ pp collisions. We take the mass of the two hardest jets found using the $\mathrm{C} / \mathrm{A}$ algorithm with $R=1.0$ and apply the algorithms with a fixed $z_{\text {cut }} / y_{\text {cut }}$.

that the action of trimming for the chosen parameters, appears only to have an apparently subleading effect and hence the desirable property of Y-splitter, that of reducing background via a Sudakov suppression term (see eq. (5.6)), is largely unaffected. Such findings are certainly worthy of analytical follow-up for general choices of parameters, which we shall provide in our forthcoming work.

Given the improvement in signal efficiency that we have achieved with Y-splitter with trimming, and the fact that the backgrounds are comparably (and in fact apparently somewhat more) suppressed compared to Y-pruning in the mass region of interest, it is worth examining the signal significances (i.e. the ratio $\varepsilon_{S} / \sqrt{\varepsilon_{B}}$ of signal efficiency to the square root of background efficiency) that can be achieved with the various taggers, as a function of transverse momentum. These are shown in figure 13 for quark and gluon backgrounds. One observes that the Y-splitter with trimming method outperforms the taggers discussed here, particularly at high $p_{T}$. Note that we have used $R_{\text {trim }}=0.3$ for Y-splitter with trimming. For trimming however this represents a non-optimal choice at high $p_{T}$ (see figure 18 later) and hence we have chosen to present our results for trimming with $R_{\text {trim }}=0.1$. A detailed study of optimal parameters for Y-splitter+trimming remains to be carried out and we shall aim to present the results of such a study in forthcoming work.

The results shown in figure 13 are for our standard process, $p p \rightarrow Z H$, but similar results are also obtained for $W$ tagging as shown in figure 14 . Here we observe that $\mathrm{Y}$ splitter with trimming now consistently outperforms the other taggers discussed over a range of $p_{T}$. This emerges from the different mass window of the $\mathrm{W}$ boson $(64-96 \mathrm{GeV})$ compared to the Higgs $(109-141 \mathrm{GeV})$. In the window $M_{W} \pm 16 \mathrm{GeV}$, the background mass distribution of Y-splitter+trimming is smaller relative to Y-pruning than the window 

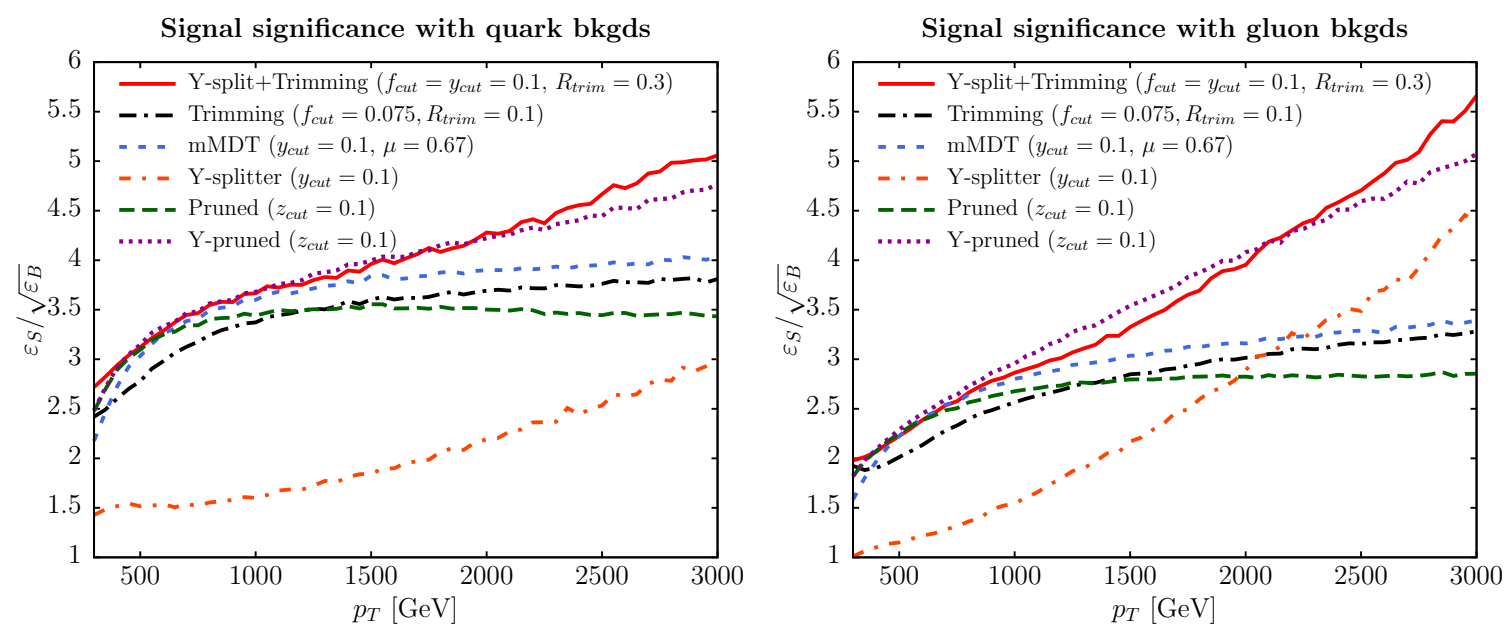

Figure 13. Signal significance for tagging hadronic $\mathrm{H}$ jets with quark (left panel) and gluon (right panel) backgrounds using Herwig ++ 2.7.0 [44] with underlying event and hadronisation as a function of a generator level cut $p_{T}$ on transverse jet momentum. We compare the signal significance for different algorithms to Y-splitter+trimming and find that the latter outperforms the others at high $p_{T}$. Here we have used $R_{\text {trim }}=0.1$ for pure trimming since, in contrast to Y-splitter+trimming, we expect this value to be closer to optimal than $R_{\text {trim }}=0.3$ at high transverse momenta (see figure 18).

around $M_{H}$ (see figure 12). Hence, we observe a greater signal significance tagging $W$ rather than Higgs relative to the other taggers for large $p_{T} \cdot{ }^{8}$

\section{Optimal parameter values}

In this section we shall use analytical expressions to derive values of parameters that maximise the signal significance $\frac{\varepsilon_{S}}{\sqrt{\varepsilon_{B}}}$ for the different taggers. We do not expect the values so derived to really be optimal in the sense that they will not take into account non-perturbative effects. Indeed we should emphasise that optimal parameter values have already been extracted using full MC studies for all methods considered in the original papers and also examined in subsequent studies such as in ref. [26]. Analytical studies of optimal parameters have also been carried out by Rubin in ref. [34] in the context of a filtering analysis, which we do not consider here.

Nevertheless we can regard it as one of the tests of the robustness of these methods that the values derived here with analytical formulae as inputs should be reasonable approximations to what one obtains in complete MC studies. This is because one wants ideally to have substructure methods where statements about performance are largely independent of our detailed knowledge about non-perturbative corrections. We are also interested in examining to what extent general trends that emerge with analytics, such as the dependence of optimal parameters on $p_{T}$, are replicated in full $\mathrm{MC}$ studies. For the following

\footnotetext{
${ }^{8} \mathrm{We}$ have performed preliminary studies for other possible combinations such as Ysplitter+mMDT/pruning/soft drop. These all have a similar qualitative effect on both the background and signal jet mass distribution as $\mathrm{Y}$-splitter+trimming. Hence, one observes a comparable gain in signal significance over $\mathrm{Y}$-splitter for all of these combinations. However, we find that $\mathrm{Y}$-splitter+trimming has the best signal significance for tagging $\mathrm{W}$ bosons over background in the high $p_{T}$ limit.
} 

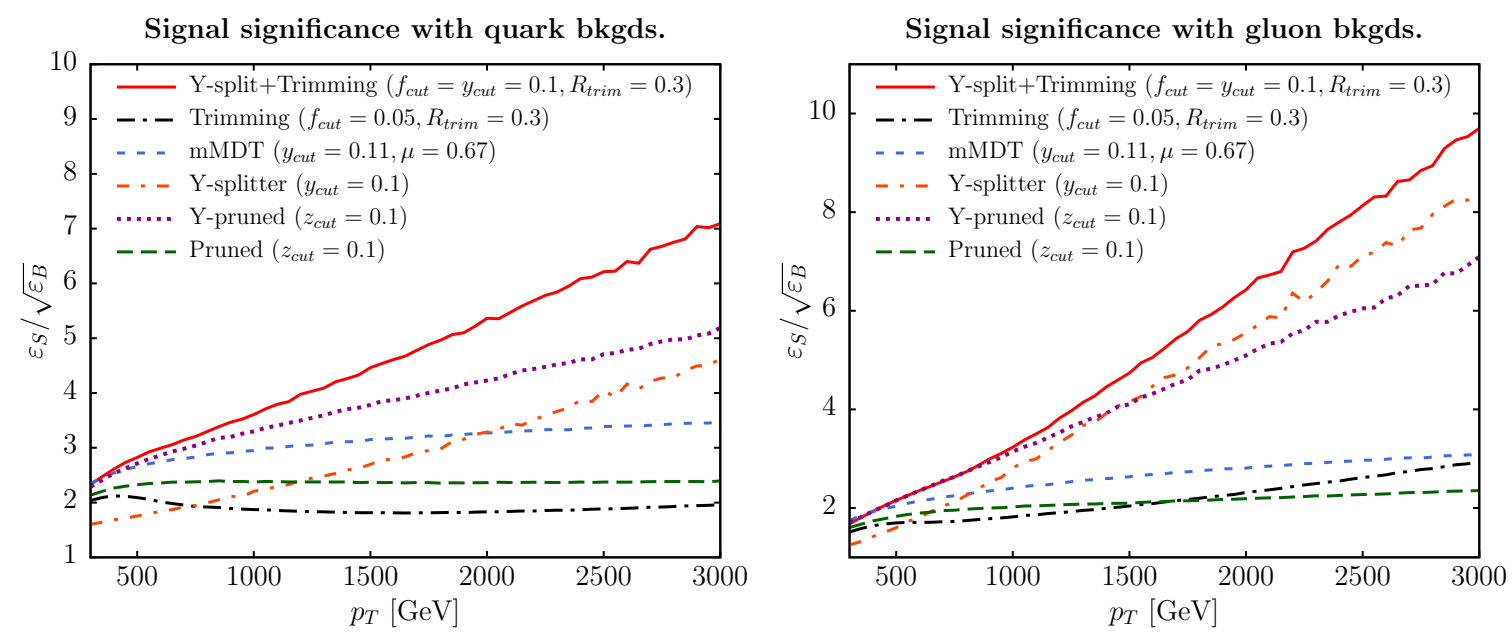

Figure 14. Signal significance for tagging hadronic W jets with quark (left panel) and gluon (right panel) backgrounds using Herwig $++2.7 .0[44]$ with underlying event and hadronisation as a function of a generator level cut $p_{T}$ on transverse jet momentum. We deem a jet tagged if it has a final mass in the window $64-96 \mathrm{GeV}$. We compare the signal significance for different algorithms to Ysplitter+trimming and find that the latter outperforms the others at high $p_{T}$. In this plot, we use all tagger parameters which match those used for $\mathrm{W}$ tagging in the paper [31] for ease of comparison.

studies we confine ourselves to quark backgrounds as we have no reason to believe that gluon backgrounds will differ significantly in terms of the conclusions we reach here.

Having observed in this paper the relatively small radiative corrections, both for ISR and FSR, that emerge for signal processes over a broad range of parameter values, one feels encouraged in a first approximation to turn off these effects and treat the signal in a treelevel approximation, except for the case of trimming as we discuss below in more detail. In other words we anticipate that the signal significance ought to primarily be driven by the tree-level results for signal while for the background we shall use the resummed formulae first derived in [31]. For self-consistency, one should then also verify that for the optimal values one derives, the radiative corrections to signal efficiency can indeed be considered small relative to the tree level result.

\section{1 mMDT}

Let us follow the above described procedure for the mMDT and extract the optimal value of $y_{\text {cut. }}$.

One needs to study the background mistag rate in the window $M_{H}-\delta M<M_{j}<$ $M_{H}+\delta M$ which corresponds to a range in $\rho, \rho_{H}-\delta \rho<\rho<\rho_{H}+\delta \rho$, with $\delta \rho \approx 2 M_{H} \delta M / p_{T}^{2}$ where we have used $R=1$.

We then have the following expression for the signal significance:

$$
\frac{\varepsilon_{S}}{\sqrt{\varepsilon_{B}}}=\frac{1-2 y_{\text {cut }}}{\sqrt{\sum\left(y_{\text {cut }}, \rho_{H}+\delta \rho\right)-\Sigma\left(y_{\text {cut }}, \rho_{H}-\delta \rho\right)}},
$$

with $\rho_{H}=\frac{M_{H}^{2}}{p_{T}^{2}}$ and where $\Sigma\left(y_{\text {cut }}, \rho\right)$ is the integrated mMDT background jet mass distribution calculated in ref. [31]. Thus the quantity $\Sigma\left(y_{\text {cut }}, \rho_{H}+\delta \rho\right)-\Sigma\left(y_{\text {cut }}, \rho_{H}-\delta \rho\right)$ represents 
the integral of the background jet-mass distribution over the mass window corresponding to signal tagging with mMDT. Note that we have treated the signal efficiency at lowest order.

We can find the value of $y_{\text {cut }}$ that maximises signal significance by taking the derivative of the r.h.s. of eq. (7.1) w.r.t. $y_{\text {cut }}$ and setting it to zero which gives:

$$
\frac{-4}{1-2 y_{\text {cut }}}=\frac{\Sigma^{\prime}\left(y_{\text {cut }}, \rho_{H}+\delta \rho\right)-\Sigma^{\prime}\left(y_{\text {cut }}, \rho_{H}-\delta \rho\right)}{\Sigma\left(y_{\text {cut }}, \rho_{H}+\delta \rho\right)-\Sigma\left(y_{\text {cut }}, \rho_{H}-\delta \rho\right)}
$$

where $\Sigma^{\prime}$ denotes a derivative w.r.t. $y_{\text {cut }}$. Neglecting higher order corrections in $\delta \rho$, the optimal value for $y_{\text {cut }}$ satisfies

$$
\frac{-4}{1-2 y_{\mathrm{cut}}}=\frac{\frac{d}{d y_{\mathrm{cut}}}\left(\frac{\partial \Sigma}{\partial \rho}\right)_{\rho=\rho_{H}}}{\left(\frac{\partial \Sigma}{\partial \rho}\right)_{\rho=\rho_{H}}} .
$$

Next we use the analytical expressions for $\Sigma\left(y_{\text {cut }}, \rho\right)$ derived in ref. [31]. The fixed-coupling result for the mMDT for $\rho<y_{\text {cut }}$ reads:

$$
\Sigma\left(y_{\text {cut }}, \rho\right)=\exp \left[-\frac{C_{F} \alpha_{s}}{\pi}\left(\ln \frac{1}{y_{\text {cut }}} \ln \frac{y_{\text {cut }}}{\rho}-\frac{3}{4} \ln \frac{1}{\rho}+\frac{1}{2} \ln ^{2} \frac{1}{y_{\text {cut }}}\right)\right] .
$$

We can use this result in eq. (7.3), assuming that the optimal value lies in the region $\rho<y_{\text {cut }},{ }^{9}$ and doing so gives us an implicit equation for optimal $y_{\text {cut }}=y_{\text {max }}$ :

$$
\frac{-4 y_{\max }}{1-2 y_{\max }}=C_{F} \frac{\alpha_{s}}{\pi} \ln \frac{y_{\max }}{\rho_{H}}+\frac{4}{3+4 \ln y_{\max }} .
$$

One can numerically solve the above equation, which contains the essential information about the optimal $y_{\text {cut }}$ and its dependence on $p_{T}$. The values we obtain for $p_{T}=1,2,3 \mathrm{TeV}$ with $\alpha_{s}=0.1$ are approximately $0.124,0.102$ and 0.088 respectively. Whilst we have not included running coupling effects in the above derivation, one finds it is straightforward to do so. Using the full calculation of ref. [31] for the background, i.e. including running coupling effects and a transition to the plain mass like behaviour for $\rho>y_{\text {cut }}$, we compute the analytical signal significance plotted in figure 15 as a function of $y_{\text {cut }}$.

From figure 15 we note firstly that the peak position of the analytical signal significance is approximately in agreement with the numbers we quoted immediately above for the fixed-coupling calculation. A kink can be seen in the analytical result for $p_{T}=1 \mathrm{TeV}$, the origin of which is the transition from a single-logarithmic dependence on $\rho$ valid at $\rho<y_{\text {cut }}$, to the usual double logarithmic result for the plain mass for $\rho>y_{\text {cut }}$. We have also shown, in the same figure, results from Herwig ++2.7 .0 at both parton level and at full hadron level including UE. We find the Herwig ++2.7 .0 results at parton level in quite reasonable agreement with the simple analytical estimates we have made, for both the peak positions and the evolution of optimal $y_{\text {cut }}$ with $p_{T}$, though the values of the peak signal significance itself differ somewhat. It is noteworthy also that hadronisation and UE

\footnotetext{
${ }^{9}$ This is reasonable at high $p_{T}$, since $\rho_{H}(1 \mathrm{TeV}) \approx 0.015$ much smaller than typical $y_{\text {cut }}$ values $\sim 0.1$ quoted in the literature.
} 

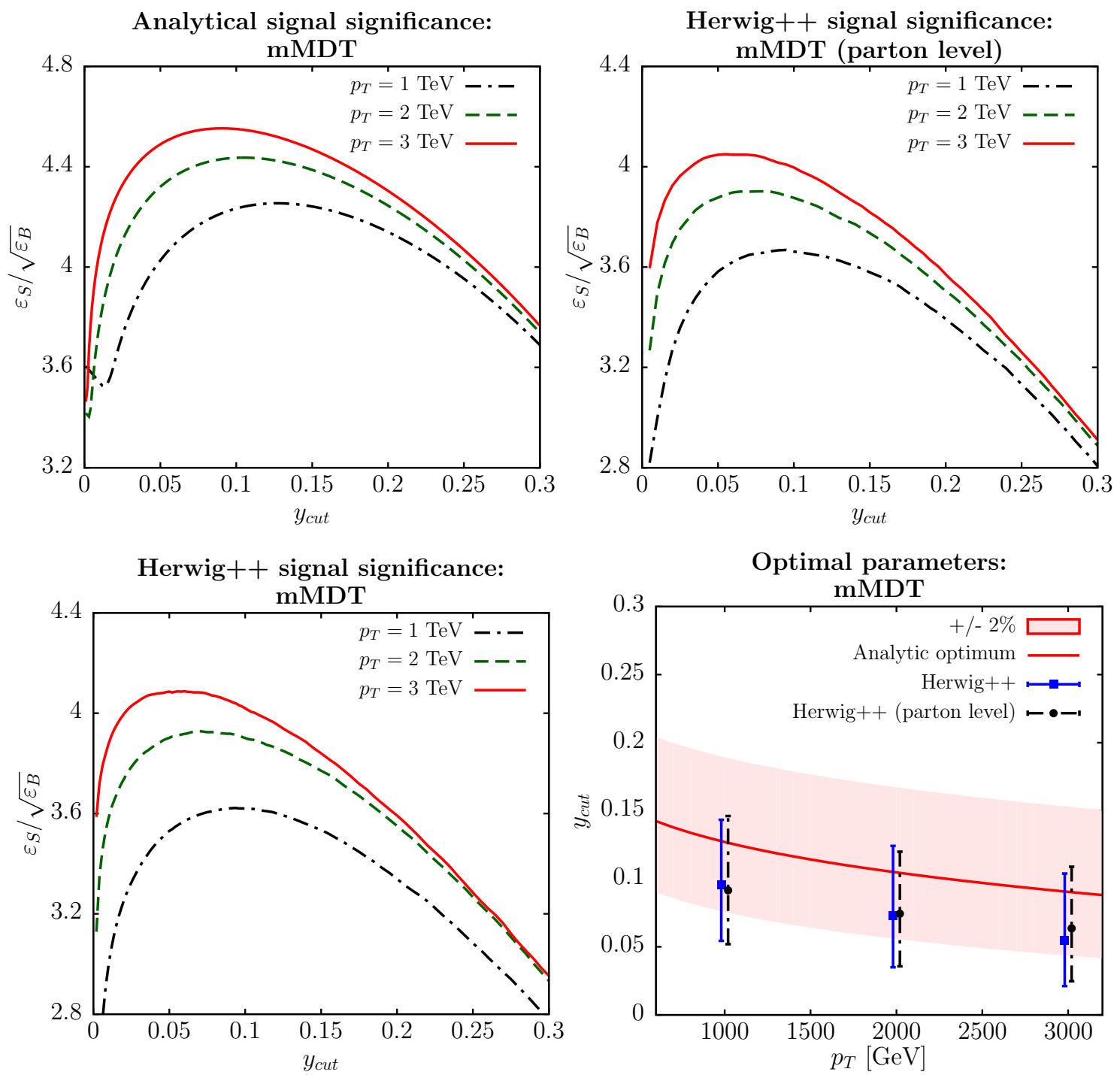

Figure 15. mMDT analytical signal significance from tree level signal and resummed background as a function of $y_{\text {cut }}$ (top left) compared to Herwig ++2.7 .0 [44] at parton level (top right) and with hadronisation and MPI (bottom left). The signal process used is $p p \rightarrow Z H$ where we require the Higgs and Z to decay hadronically and leptonically respectively with quark backgrounds. We place a generator level cut on the Higgs transverse momentum $p_{T}$ of 1,2 and $3 \mathrm{TeV}$. Jets are tagged around the Higgs mass with a mass window $\delta M=16 \mathrm{GeV}$. The bottom right panel shows the analytic optimal $y_{\text {cut }}$ values as a function of $p_{T}$ (red line) with a $2 \%$ variation in signal significance about the peak (red shaded area). We overlay the optimal results for $y_{\text {cut }}$ obtained using Herwig ++2.7 .0$ with hadronisation and underlying event at 1, 2 and $3 \mathrm{TeV}$, with an equivalent $2 \%$ variation about the peak signal significance (blue bars) and at parton level (black bars). 
do not change the picture significantly at the $p_{T}$ values we have studied here. One other feature that emerges from both analytical and MC studies is that the peaks themselves are fairly broad so that choosing a slightly non-optimal $y_{\text {cut }}$ does not greatly impact the tagger performance. We have also provided in figure 15 a direct comparison between optimal values from Herwig ++2.7 .0 (including all effects) and analytical estimates. We show the results for the range of $y_{\text {cut }}$ values (denoted by the pink shaded region) that correspond to a $\pm 2 \%$ variation around the peak signal significance. For Herwig ++2.7 .0 instead we indicate the same range of $y_{\text {cut }}$ values by the blue bars shown. We find a good degree of overlap within this tolerance band between full Herwig ++2.7 .0 results and analytical estimates.

One can draw at least a couple of inferences from our observations above. Firstly, as we have argued, radiative corrections to the signal are clearly of minor significance to the tagger performance for mMDT. The fact that the analytics are generally in good agreement with Herwig ++2.7 .0 points to the importance of the background contribution in the context of the signal significance and the success of analytical approaches in describing this background [31]. The fact that non-perturbative effects play an evidently minor role at the values of $p_{T}$ studied above is also reassuring from the point of view of a robust understanding of tagger performance.

We end with a caveat. If one moves to still lower $p_{T}$ values then one has to reconsider some of the arguments above. Here one would have a situation where say at $200-300 \mathrm{GeV}$ we expect to be in the region where $\rho>y_{\text {cut }}$ and so eq. (7.5) does not directly apply. This apart, perhaps more significantly one can expect UE to start playing a larger role due to the larger effective radius $\sim \frac{M_{j}}{p_{T}}$ where UE particles accumulate without being removed by the asymmetry cut. Here one ought to consider the use of mMDT with filtering and optimise the parameters of both methods together as in the original analysis [3].

\subsection{Pruning and Y-pruning}

Here we carry out a similar analysis for the case of pruning. The resummed expression for pruning, for QCD jets, is considerably more complicated than for mMDT. The result essentially has two components which in ref. [31] were dubbed the Y and I components respectively. We have already dealt with Y-pruning in some detail in this article in the context of signal jets. For the background, as we have also discussed in a previous section, Y-pruning, for small jet masses, $\rho<z_{\text {cut }}^{2}$, consists of a suppression of the leading order single-logarithmic result by a Sudakov like form factor and gives rise to a desirable suppression of the background in the signal region, for high $p_{T}$ values. The I pruning contribution, on the other hand, starts at order $\alpha_{s}^{2}$ and is as singular as the plain jet mass i.e. double logarithmic. For the sum of $\mathrm{Y}$ and I components, i.e. for pruning as a whole, one observes two transition points: for $\rho>z_{\text {cut }}$ the behaviour is like the plain mass (as for mMDT), for $z_{\text {cut }}^{2}<\rho<z_{\text {cut }}$ there is a single log behaviour as in the leading-order result and as for mMDT, while for $\rho<z_{\text {cut }}^{2}$ we see the I-pruning contribution starts to become more important which can cause growth of the background and the appearance of a second peak for quark jets and a shoulder like structure for gluon jets. 

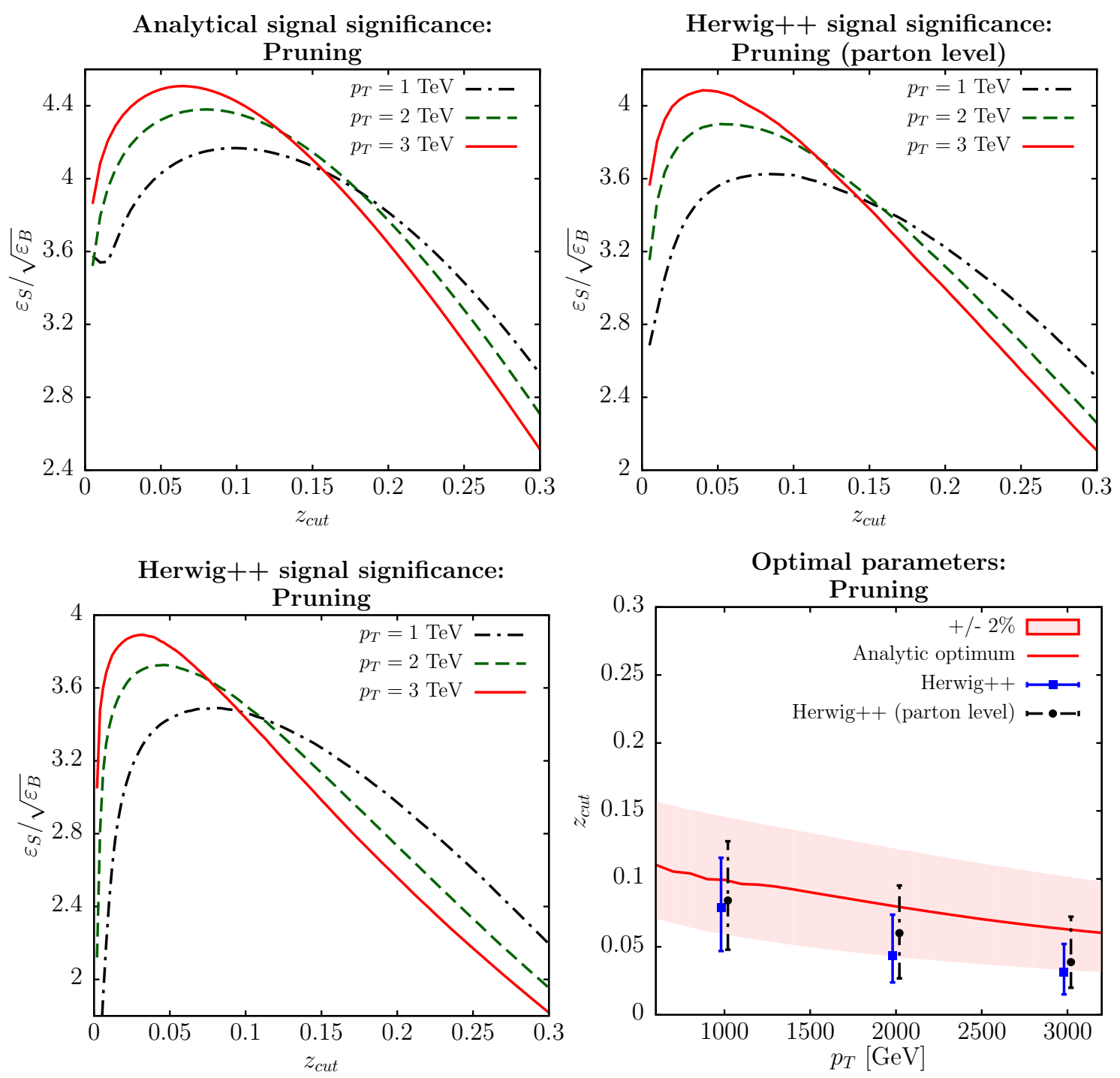

Figure 16. Pruning analytical signal significance from tree level signal and resummed background as a function of $z_{\text {cut }}$ compared to Herwig ++2.7 .0 at parton level and with hadronisation and MPI. Details of generation given in figure 15 .

We do not, for brevity, present here the resummed results for pruning for QCD jets, referring the reader instead to section 5.3 of ref. [31]. Here we simply plot the analytical signal significance for pruning as for $\mathrm{mMDT}$, with neglect of radiative corrections to the signal efficiency, but with the full resummed calculation for QCD background, which we take to be quark jets alone. The resulting signal significance is displayed in figure 16 along with MC results at parton and hadron level. One would expect the optimal $z_{\text {cut }}$ to lie in a region that corresponds to the mMDT like region i.e. such that $\rho_{H}>z_{\text {cut }}^{2}$. Choosing a larger $z_{\text {cut }}$ would push us into the region where the background starts to grow due to onset of I-pruning and hence the signal significance falls off. At $1 \mathrm{TeV}$ where $\rho_{H} \approx 0.0017$ one can expect an optimal value of $z_{\text {cut }}$ to be below $\sqrt{\rho_{H}} \approx 0.125$ while for $3 \mathrm{TeV}$ one 
may expect a value closer to 0.04 and these expectations are roughly consistent with what one notes with both the analytical and MC results shown. Once again we observe that non-perturbative effects do not change the essential picture one obtains from analytics and have only a limited impact on the signal significance relative to parton level.

The pruning results have clear qualitative differences from the case of mMDT. In particular at higher $p_{T}$ we have to be more precise about the choice of $z_{\text {cut }}$ due to the somewhat narrower peak in the signal significance. We can compare, as for mMDT, analytical results to those from Herwig ++2.7 .0 , once again with a $\pm 2 \%$ tolerance band shown in the bottom right figure of figure 16 . We observe that within this small tolerance band the results are compatible though at higher $p_{T}$ perhaps less so than for mMDT.

In the original paper [20], the authors conclude that the optimal $z_{\text {cut }}$ value for pruning is 0.1 when using the $\mathrm{C} / \mathrm{A}$ algorithm to cluster the initial jet, as we do here. This optimisation was performed at a moderate transverse momenta $(100-500 \mathrm{GeV}$ for $\mathrm{W}$ bosons) compared to this paper, however our results are consistent as we approach this region. For larger boosts, we observe that the optimal value choice for $z_{\text {cut }}$ tends to slightly smaller values $\left(z_{\text {cut }} \sim 0.075\right)$.

We also present in figure 17 results for the signal significance of Y-pruning, again taking quark jets as background. Here we note firstly that analytics are again broadly in agreement with MC results for the shape of the signal significance as a function of $z_{\text {cut }}$. Secondly the peaks are quite broad and so choosing a somewhat non-optimal value of $z_{\text {cut }}$ does not critically affect the significance. Furthermore, the optimal $z_{\text {cut }}$ does not depend strongly on $p_{T}$ and is virtually constant over the limited $p_{T}$ range studied. Lastly within a $\pm 2 \%$ tolerance band there is good agreement between full $\mathrm{MC}$ results and simple analytics on the optimal values of $z_{\text {cut }}$.

Hence for mMDT and Y-pruning and to a slightly smaller degree for pruning we find that, over the $p_{T}$ values we studied here, analytical results based on resummed calculations for QCD background and lowest order results for signals, with neglect of non-perturbative effects, capture the essential features of tagger performance, as reflected in the signal significance. An extension of our studies to lower $p_{T}$ values would be of interest in order to ascertain the further validity of the simple picture we have used for our analytical results and probe in more detail the role of radiative corrections to the signal and that of non-perturbative contributions. We shall next examine the more involved case of optimal parameters for trimming.

\subsection{Trimming}

Here we carry out a similar analysis for trimming, but one now has to optimise two parameters, $R_{\text {trim }}$ and $f_{\text {cut }}$. As performed for the pruning analysis, we use the analytic resummed expression for QCD jets given in ref. [31]. The result for the background jet mass distribution consists of a region with single log behaviour (equivalent in structure to mMDT) for $f_{\text {cut }} R_{\text {trim }}^{2}<\rho<f_{\text {cut }}$ which transitions into a double logarithmic growth in the background distribution for $\rho<f_{\text {cut }} R_{\text {trim }}^{2}$. However, in contrast to mMDT and (Y-)pruning, FSR radiative corrections to the signal efficiency are crucial for optimisation. If one naively uses the tree level result given in eq. (3.2), it follows that the optimum value for $R_{\text {trim }}$ tends to zero. 

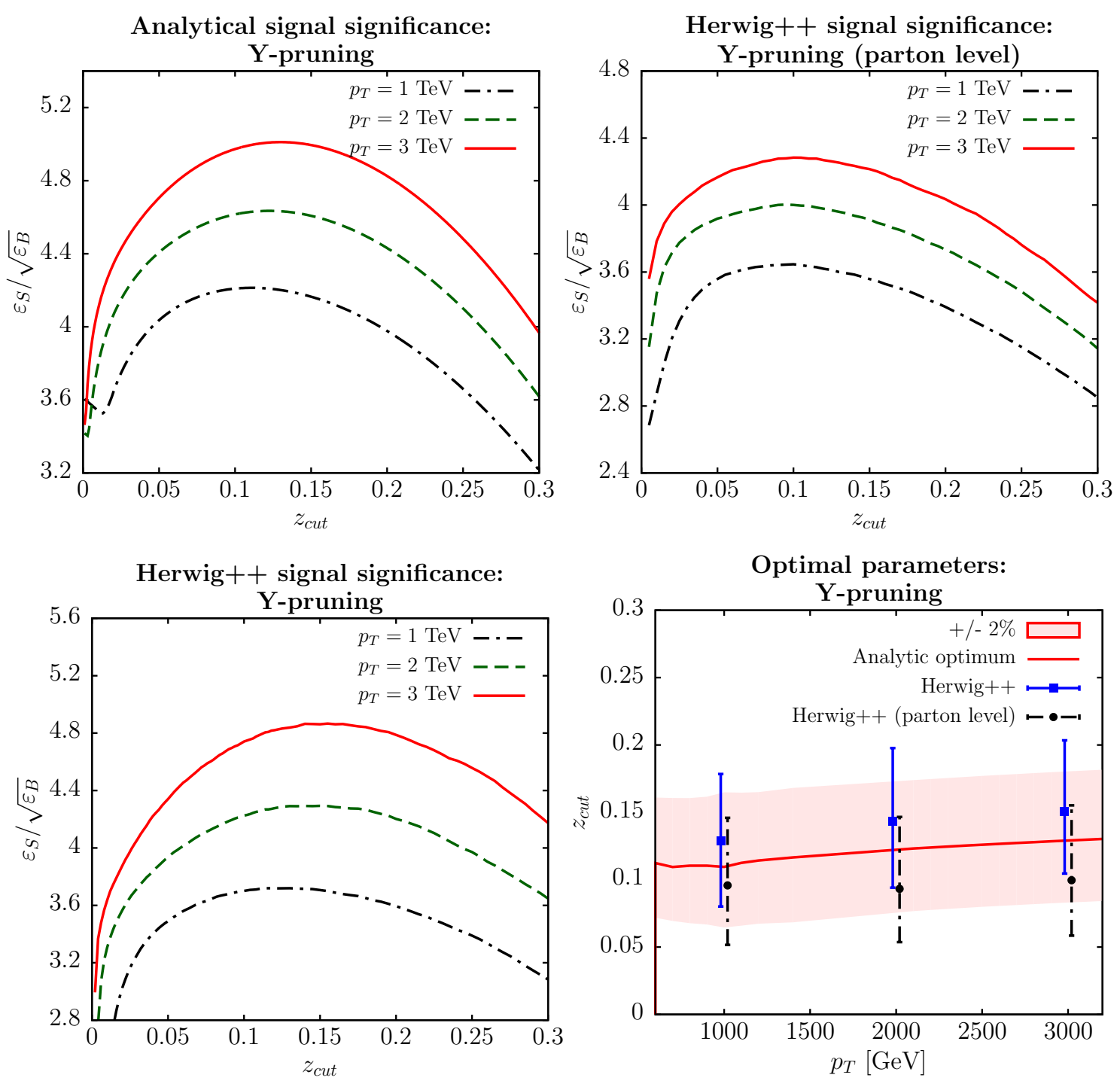

Figure 17. Y-pruning analytical signal significance from tree level signal and resummed background as a function of $z_{\text {cut }}$ compared to Herwig ++2.7 .0 at parton level and with hadronisation and MPI. Details of generation given in figure 15.

This is because one can ensure that signal mass window is within the single logarithmic region by simply pushing the location of the double logarithmic transition $\left(\rho=f_{\text {cut }} R_{\text {trim }}^{2}\right)$ to small values of the QCD jet mass, thereby avoiding the double logarithmic peak.

However, as shown in this paper, in the limit $R_{\text {trim }} \rightarrow 0$, one encounters large logarithmic corrections to the signal efficiency associated with final state radiation from the signal jet (see eq. (3.11)). This puts a limit on how small one can reduce the trimming radius whilst maintaining reasonable signal mass resolution. Hence, we now include FSR radiative corrections to the signal efficiency by integrating the the expression given in eq. (3.10) over $z$ and adding this term to the Born level result eq. (3.2). Including this radiative correction, along with the resummed QCD background, we can obtain analytical estimates for the signal significance. 

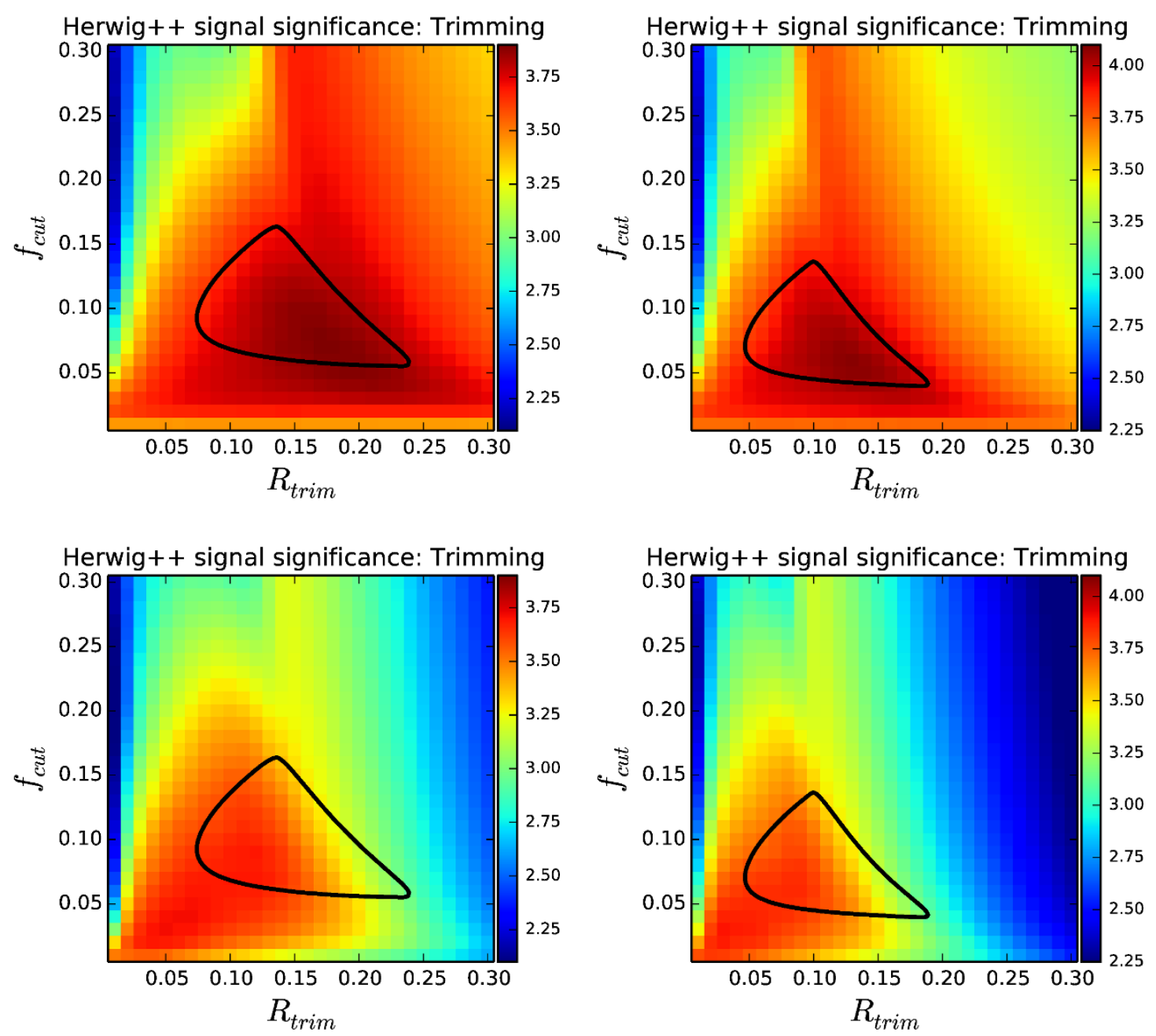

Figure 18. 2D density plot showing the trimming signal significance as a function of $R_{\text {trim }}$ and $f_{\text {cut }}$ using Herwig ++2.7 .0 for $H \rightarrow b \bar{b}$ jets with quark backgrounds with a minimum jet transverse momentum cut. The top panels are generated at parton level with transverse momenta $2 \mathrm{TeV}$ and $3 \mathrm{TeV}$ left and right and the bottom panels include hadronisation and underlying event. The area inside the black contour represents the analytic prediction with FSR radiative corrections to the signal efficiency for optimal values within $2 \%$ of the analytic peak signal significance.

In figure 18 we show a 2D density plot for the signal significance with trimming over a range of $R_{\text {trim }}$ and $f_{\text {cut }}$ values using Monte Carlo at parton level (top) and with full hadronisation and underlying event (bottom) with a transverse momentum cut at 2 and $3 \mathrm{TeV}$ left and right respectively. We overlay a black analytical contour representing the region in which the analytical signal significance is no more than $\pm 2 \%$ away from the analytically derived peak value for $R_{\text {trim }}$ and $f_{\text {cut }}$. One can see that we have reasonable agreement between the simple analytical estimates and the Herwig ++2.7 .0 results at parton level. However, when one includes non-perturbative effects, we observe that contamination from underlying event significantly reduces the signal significance as $R_{\text {trim }}$ increases. 

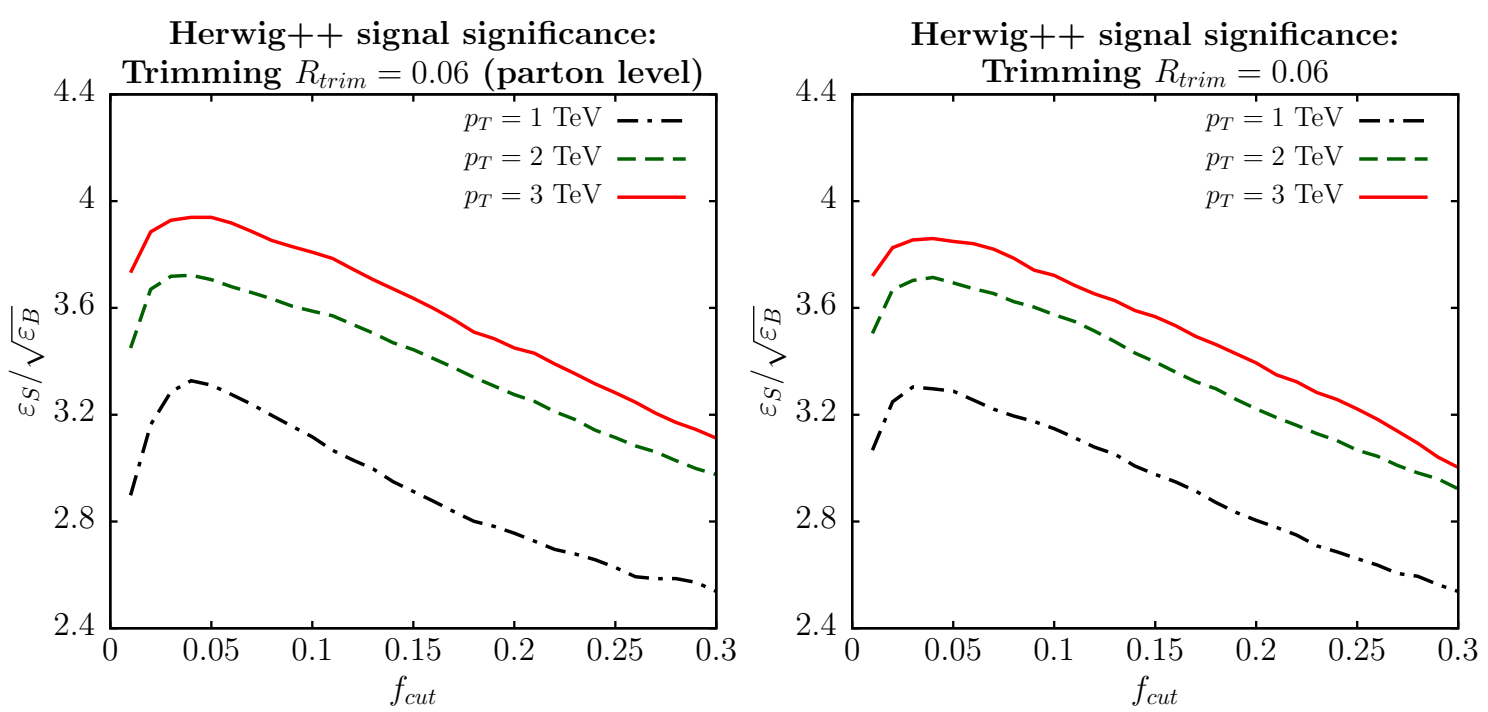

Figure 19. Trimming signal significance for $R_{\text {trim }} \sim \sqrt{\Delta} \sim 0.06$ as a function of $f_{\text {cut }}$ generated at parton level and with hadronisation and MPI using Herwig++ 2.7.0.

We can use our simple analytical estimates to comment on the optimal values we observe from MC. Firstly, for optimal values of $f_{\text {cut }}$ and $R_{\text {trim }}$, one would expect the signal mass window to reside in the single logarithmic mMDT-like region of the background, hence we anticipate that the optimal parameters satisfy the constraint $\Delta>f_{\text {cut }} R_{\text {trim }}^{2}$. This expectation is consistent with both the analytical contour (top right edge) and MC results both at parton and full hadron level. This background driven effect is manifest as a suppression in signal significance when the product $f_{\text {cut }} R_{\text {trim }}^{2}$ becomes large (i.e top right of the contour plots). For example, at $3 \mathrm{TeV}$ and fixed $f_{\text {cut }}=0.1$, one would analytically expect an optimal value for $R_{\text {trim }} \lesssim 0.13$, whilst at $f_{\text {cut }}=0.05$ one expects $R_{\text {trim }} \lesssim 0.19$. These numbers are in agreement with the analytical contour and MC results. Secondly, FSR corrections to the signal efficiency become significant in the region $R_{\text {trim }}^{2} \ll \Delta$, hence one would expect the optimal trimming radius to reside in the region $R_{\text {trim }} \gtrsim \sqrt{\Delta}$. At $3 \mathrm{TeV}$ this corresponds to $R_{\text {trim }}>0.04$ and at $2 \mathrm{TeV}$ corresponds to $R_{\text {trim }}>0.06$. This is consistent with the analytical contour and MC, where we observe a reduction in signal significance in the limit $R_{\text {trim }} \ll \sqrt{\Delta}$.

We notice that, like mMDT and pruning, the signal significance is fairly insensitive to variations in $f_{\text {cut }}$ provided we choose $R_{\text {trim }}$ such that $\sqrt{\Delta / f_{\text {cut }}}>R_{\text {trim }}$. However, the signal significance is subject to non-perturbative corrections which increase with $R_{\text {trim }}$, and consequently one should favour the small $R_{\text {trim }}$ limit of the analytical optimal contour region $R_{\text {trim }} \approx \sqrt{\Delta}$ to minimise both signal FSR and non-perturbative corrections to the signal significance. It is thus of interest to choose $R_{\text {trim }}^{2} \sim \Delta$ and study the dependence of the signal significance on the choice of $f_{\text {cut }}$ as in figures $15,16,17$. The results can be found in figure 19 for $R_{\text {trim }}=0.06$ which is identical to $\Delta$ at $2 \mathrm{TeV}$ and of order $\Delta$ for the other $p_{T}$ values. With the given choice of $R_{\text {trim }}$, reminiscent of the pruning radius, it is natural to compare the results to those for pruning reported in figure 16. One notes that even 
with a similar choice of radius there are differences between the two techniques. While for pruning the optimal $z_{\text {cut }}$ decreases with increasing $p_{T}$ the optimal value for trimming stays more constant. The peak signal significance itself increases with $p_{T}$ in both cases. For a given $p_{T}$ the behaviour as a function of $f_{\text {cut }}$ is also different, especially at larger $f_{\text {cut }}$. These differences originate in a number of sources: the difference in FSR corrections and their $p_{T}$ dependence which is more pronounced for trimming, differences in the definitions of $f_{\text {cut }}$ and $z_{\text {cut }}$ and last but not least differences arising from QCD background jets with pruning and trimming (see ref. [31]). In order to better understand the role for example of FSR effects, in the above context, we note that for pruning one can simply replace the signal efficiency by $1-2 z_{\text {cut }}$ as we have done for our analytical studies of optimal parameter values in pruning. If one similarly uses $1-2 f_{\text {cut }}$ in computing the signal efficiency for trimming one observes that the result with $R_{\text {trim }}^{2} \sim \Delta$ is closer to that for pruning and optimal $f_{\text {cut }}$ values show a very similar trend with $p_{T}$ to those for pruning. However for trimming $p_{T}$ dependent FSR corrections cannot be neglected, especially at low $p_{T}$, and play an important role in pushing the optimal $f_{\text {cut }}$ to smaller values than would be obtained by turning off FSR effects. This is the main reason behind the relative insensitivity of optimal $f_{\text {cut }}$ values seen with trimming, over the $p_{T}$ range studied in figure 19.

\section{Conclusions}

In this article we have studied perturbative radiative corrections and non-perturbative effects for the case of signal jets, specifically for boosted Higgs production with $H \rightarrow b \bar{b}$, with the application of jet substructure taggers. For the former we have carried out relatively simple analytical calculations both to assess the impact of ISR and FSR as well as to study its dependence on various parameters, such as a mass window of width $\delta M$ on either side of the signal mass, the fat jet $p_{T}$, the mass of the resonance $M_{H}$, and the parameters of the various taggers. To examine non-perturbative effects we have confined ourselves to MC studies.

Our study was motivated by relatively recent calculations dedicated to the case of QCD background jets and in particular work presented in ref. [31]. There it was noted that while taggers should in principle discriminate against jets from QCD background, the degree to which this happened and the impact on the background jet mass distribution was not always as desired. While taggers such as pruning, mMDT and trimming were essentially identical over a limited range in the normalised square jet mass $\rho=M_{j}^{2} /\left(p_{T}^{2} R^{2}\right)$, significant differences in performance and behaviour were observed at small values of $\rho$, which especially at high $p_{T}$ corresponded to masses in the signal mass region of interest. Likewise taggers should, in principle, not affect significantly signal jets, retaining them as far as possible. Additionally most taggers have a grooming element (via the $f_{\text {cut }} / y_{\text {cut }} / z_{\text {cut }}$ criteria) that is responsible for clearing the jet of contamination from ISR/UE thereby helping in the reconstruction of sharper mass peaks. Here our aim was to carry out analytical and MC studies to investigate in detail the impact of taggers on signal especially with regard to the interplay between tagger parameters as well as kinematic cuts such as jet $p_{T}$, masses and mass windows. 
Our findings on the whole indicate that tagger performance is more robust for the case of signal jets than was apparent for QCD background. Most taggers are quite similar in their response to ISR and generally significantly ameliorate the loss of the signal efficiency seen for plain jet mass cuts, without these substructure techniques. An exception to this situation was the case of Y-splitter where the ISR and UE contamination resulted in a loss of signal efficiency identical to that seen for plain jets.

Likewise for FSR, the radiative losses that one sees are on the whole modest for a reasonably wide range of tagger parameters. Here an interesting question opens up about the potential role for fixed-order calculations in the context of jet substructure studies. This is because one observes an absence of genuine logarithmic enhancements for sensibly chosen tagger parameter values. The signal efficiency, for the taggers studied here, ought then to be better described by exact calculations that incorporate hard gluon radiation or by combinations of matrix element corrections and parton showers than by the soft/collinear emissions encoded in pure parton showers. We carried out a comparison between an MC description of the signal efficiency and exact order $\alpha_{s}$ results for various taggers, reported in appendix B. We find that we can reasonably adjust parameters such as the size of our mass window $\delta M$ to obtain good agreement between the two descriptions. Such observations may also be useful beyond the immediate context of our work, in situations where differences in tagger performance could come from regions of phase space that are not under the control of a soft eikonal approximation. In these situations one would ideally want to combine resummed calculations, where necessary, with fixed-order calculations i.e. carry out matched resummed calculations. A summary of the results presented in this paper for the logarithmic structure of radiative corrections to the signal efficiency for each tagger are given in table. 1.

A development we have made here is the introduction of a combination of Y-splitter with trimming in an attempt to improve the response of Y-splitter to ISR/UE contamination. The main reason why we made this effort was due to the fact that we observed that Y-splitter was very effective at suppressing QCD background in the signal region. The resulting improvement of signal efficiency coupled with the fact that the background suppression from Y-splitter remained essentially intact after the use of trimming, meant that the combination of Y-splitter+trimming actually outperforms other taggers studied here, in particular, at high $p_{T}$. Our observation is in keeping with the general idea that suitably chosen tagger combinations may prove to be superior discovery tools compared to currently proposed individual methods [64]. In fact it is now becoming increasingly common to use combinations of techniques such as N-subjettiness [62] with for instance mMDT in an effort to maximise tagger performance (see e.g. [65]). There is also much effort aimed at better understanding tagger correlations and we expect that our forthcoming analytical calculations for the case of Y-splitter with trimming will shed further light on some of these issues [63].

Lastly, we have carried out an analytical study of optimal parameter values for various taggers. Having observed modest radiative corrections to the signal we neglected these effects and found that analytical estimates, based on lowest order results for the signal and resummed calculations for QCD background, generally provide a good indicator of the dependence of signal significance on the tagger parameters. The analytical formulae 


\begin{tabular}{|l|cc|}
\hline Tagger & ISR & FSR \\
\hline Plain & $R^{2} \ln \frac{R^{2}}{\epsilon \Delta}$ & $\Delta \ln \Delta$ \\
Trimming & $R^{2} \ln \frac{1}{f_{\text {cut }}}$ & $2 \ln \frac{\Delta}{R_{\text {trim }}^{2}} C_{2}\left(f_{\text {cut }}, \epsilon\right)$ \\
Pruning & $R^{2} \ln \frac{1}{z_{\text {cut }}}$ & $\frac{2 \pi}{\sqrt{3}} \ln \frac{z_{\text {cut }}}{\epsilon}$ \\
Y-pruning & $R^{2} \ln \frac{z_{\text {cut }} R^{2}}{\Delta}$ & $\frac{2 \pi}{\sqrt{3}} \ln \frac{z_{\text {cut }}}{\epsilon}$ \\
mMDT & $R^{2} \ln \frac{1}{y_{\text {cut }}}$ & $0.646 \ln \frac{y_{\text {cut }}}{\epsilon}$ \\
Y-splitter & $R^{2} \ln \frac{R^{2}}{\epsilon \Delta}$ & $\mathcal{O}\left(y_{\text {cut }}\right)$ \\
\hline
\end{tabular}

Table 1. A table summarising the logarithmic structure of radiative corrections to the signal efficiency for each tagger. For each tagger we show the coefficient of $-\frac{\alpha_{s} C_{F}}{\pi}$ for ISR and FSR results in the small $\Delta$ and $z_{\text {cut }} / y_{\text {cut }} / f_{\text {cut }}$ limit. We have defined $\epsilon=2 \delta M / M_{H}$ as in the main text. The coefficient $C_{2}$ for the trimming FSR logarithm is given in eq. (3.12).

which also do not include non-perturbative effects give rise to optimal values that are fairly compatible with those produced by full MC studies. This is encouraging from the point of view of robustness of the various methods considered since a dependence of optimal values on MC features (hadronisation models or MC tunes) are potentially not ideal.

We note in closing that for other methods, such as N-subjettiness for example, there will also be a suppression of signal jets due to the fact that such observables directly restrict radiation from the signal prongs. Thus in those cases radiative corrections arising from soft/collinear emissions by signal prongs are highly significant as can be noted from ref. [35]. We hope that our work taken together with studies of such observables will enable a more complete understanding of features of signal jets in the context of jet substructure studies and provide yet stronger foundations for future developments.

\section{Acknowledgments}

We are particularly grateful to Gavin Salam and Mike Seymour for illuminating discussions on the topic of jet substructure. We also thank Simone Marzani and Gregory Soyez for further useful discussions. We thank an anonymous referee for helpful remarks and suggestions which we have implemented in the current version of the article. We are also grateful to the Cloud Computing for Science and Economy project (CC1) at IFJ PAN (POIG 02.03.0300-033/09-04) in Cracow whose resources were used to carry out most of the numerical calculations for this project. Thanks also to Mariusz Witek and Miłosz Zdybał for their help with CC1. This work was funded in part by the MCnetITN FP7 Marie Curie Initial Training Network PITN-GA-2012-315877. We would like to thank the U.K.'s STFC for 
financial support. This work is supported in part by the Lancaster-Manchester-Sheffield Consortium for Fundamental Physics under STFC grant ST/L000520/1.

\section{A Angular integration for FSR}

To work out the coefficient of the soft FSR we need to perform the angular and $z$ integrals for the antenna pattern in eq. (3.7) for trimming and likewise for all taggers. Generally, for a single gluon emission, one has to evaluate the contribution from FSR emission outside two cones of radius $r$ centred on the $b$ and $\bar{b}$ quarks. The choice of $r$ depends on the tagger in question, so after carrying out the angular integration, one can set $r^{2}$ as $R_{\text {trim }}^{2}$ for trimming, $\Delta$ for pruning and $\theta_{b \bar{b}}^{2}=\Delta /(z(1-z))$ for mMDT and lastly integrate over $z$.

One has to then evaluate the integral

$$
I=\int \frac{d \Omega}{2 \pi} \frac{1-\cos \theta_{b \bar{b}}}{\left(1-\cos \theta_{b k}\right)\left(1-\cos \theta_{\bar{b} k}\right)} \Theta\left(\theta_{b k}^{2}-r^{2}\right)\left(\theta_{\bar{b} k}^{2}-r^{2}\right),
$$

where we have now explicitly written the conditions for the gluon to be at an angle $\theta^{2}>r^{2}$ w.r.t. both hard partons. ${ }^{10}$ The simplest way to evaluate the integral above is to first consider an integration over the entire solid angle and then to remove the contribution from inside two cones around the hard parton directions. We shall assume that the cones do not overlap, so shall consider $r<\theta_{b \bar{b}} / 2$. For larger $r$, as appropriate for mMDT where $r=\theta_{b \bar{b}}$, we perform a numerical calculation and find that our results agree with those of Rubin [34].

Therefore we write

$$
I=I_{\text {all }}-I_{\mathcal{C}_{b}}-I_{\mathcal{C}_{\bar{b}}}
$$

where $I_{\text {all }}$ is the integration over the full solid angle and $I_{\mathcal{C}_{b, \bar{b}}}$ are the integrals inside the region corresponding to cones around $b$ and $\bar{b}$ directions respectively. $I_{\text {all }}$ can be evaluated by standard techniques and yields, after azimuthal averaging, the textbook result corresponding to angular ordering of soft emission.

$$
I_{\text {all }}=\int d\left(\cos \theta_{b k}\right) \frac{\Theta\left(\theta_{b \bar{b}}^{2}-\theta_{b k}^{2}\right)}{1-\cos \theta_{b k}}+\int d\left(\cos \theta_{\bar{b} k}\right) \frac{\Theta\left(\theta_{b \bar{b}}^{2}-\theta_{\bar{b} k}^{2}\right)}{1-\cos \theta_{\bar{b} k}} .
$$

The contribution inside the cone around $b, I_{\mathcal{C}_{b}}$, can be evaluated as follows. Taking the $b$ direction as the " $z$ " axis we define the parton directions by the unit vectors:

$$
\begin{aligned}
& \vec{n}_{b}=(0,0,1) \\
& \vec{n}_{\bar{b}}=\left(0, \sin \theta_{b \bar{b}}, \cos \theta_{b \bar{b}}\right), \\
& \vec{n}_{k}=\left(\sin \theta_{b k} \sin \phi, \sin \theta_{b k} \cos \phi, \cos \theta_{b k}\right) .
\end{aligned}
$$

The in-cone subtraction term for $\mathcal{C}_{1}$ can then be written as

$$
I_{\mathcal{C}_{b}}=\int \frac{d \phi}{2 \pi} d\left(\cos \theta_{b k}\right) \frac{1-\cos \theta_{b \bar{b}}}{\left(1-\cos \theta_{b k}\right)\left(1-\cos \theta_{b \bar{b}} \cos \theta_{b k}-\sin \theta_{b k} \sin \theta_{b \bar{b}} \cos \phi\right)} \Theta\left(r^{2}-\theta_{b k}^{2}\right) .
$$

\footnotetext{
${ }^{10}$ While we have retained, at this stage, the full angular antenna pattern for ease of comparison to standard formulae, we shall later take the small angle approximation to compute the final answer.
} 
Integrating over the azimuthal angle $\phi$ gives

$$
I_{\mathcal{C}_{b}}=\int d\left(\cos \theta_{b k}\right) \frac{1-\cos \theta_{b \bar{b}}}{\left(1-\cos \theta_{b k}\right)\left|\cos \theta_{b k}-\cos \theta_{b \bar{b}}\right|} \Theta\left(r^{2}-\theta_{b k}^{2}\right) .
$$

This term can be combined with the corresponding contribution (the first term) in $I_{\text {all }}$, and taking the small-angle approximation for $\cos \theta \approx 1-\theta^{2} / 2$ ! one obtains

$$
I=\int_{0}^{r^{2}} d \theta_{b k}^{2}\left(\frac{1}{\theta_{b k}^{2}}-\frac{\theta_{b \bar{b}}^{2}}{\theta_{b k}^{2}\left(\theta_{b \bar{b}}^{2}-\theta_{b k}^{2}\right)}\right)+\int_{r^{2}}^{\theta_{b \bar{b}}^{2}} \frac{d \theta_{b k}^{2}}{\theta_{b k}^{2}}+b \leftrightarrow \bar{b}
$$

where we have also included $I_{\mathcal{C}_{\bar{b}}}$ via the interchange $b \leftrightarrow \bar{b}$. The collinear divergence along each hard parton direction is cancelled by the in-cone contributions, leaving only a wide-angle contribution. Carrying out the angular integrations we get

$$
I=2 \log \left(\frac{\theta_{b \bar{b}}^{2}-r^{2}}{r^{2}}\right),
$$

which agrees with the result found by Rubin [34] written in terms of the variable $\eta=\frac{r}{\theta_{b \bar{b}}}$, for $\eta<\frac{1}{2}$. In the collinear limit, $r \ll \theta_{b \bar{b}}$, we get the result for trimming quoted in the main text and used in eq. (3.10). To obtain the result for pruning we substitute $\theta_{b \bar{b}}^{2}=\Delta /(z(1-z))$ and $r^{2}=\Delta$, then carry out the $z$ integral, which gives:

$$
I=2 \int_{z_{\mathrm{cut}}}^{1-z_{\mathrm{cut}}} d z \ln \left(\frac{1-z(1-z)}{z(1-z)}\right)=\frac{2 \pi}{\sqrt{3}}+\mathcal{O}\left(z_{\mathrm{cut}}\right)
$$

which corresponds to the result quoted for pruning in eq. (4.4).

For mMDT where $r=\theta_{b \bar{b}}$ our calculation above, which assumed non-overlapping cones around the $b$ and $\bar{b}$, does not apply. For this purpose we have evaluated the angular integration numerically and for $M_{H} / p_{T} \ll 1$ i.e. when one can use the small-angle approximation, the result is $\approx 0.646$ as found by Rubin for the corresponding quantity $J(1)$.

\section{B Fixed-order results vs parton showers for FSR corrections}

We have noted that FSR computed using the soft approximation gives numerically very small corrections to the leading-order results, for sensible choices of the mass window $\delta M$, and the tagger parameters $y_{\text {cut }}, z_{\text {cut }}, f_{\text {cut }}$ and $R_{\text {trim }}$. This of course means that such calculations are not a good guide to the actual tagger performance i.e. the signal efficiency, since they do not produce genuine logarithmic enhancements. One can expect instead that fixed-order calculations, with correct treatment of hard non-collinear radiation at order $\alpha_{s}$ and beyond, will provide a better picture of the behaviour of taggers. Given that resummation effects are not likely to be significant it becomes of interest to compare signal efficiencies obtained with pure fixed-order calculations to those from MC generators. One may anticipate that precise order $\alpha_{s}$ calculations give quite similar results to full MC parton showers, owing to the dominance of hard radiation and the consequent lack of importance of multiple soft/collinear emissions. 
SHERPA shower vs EVENT2: mMDT

$\delta M[\mathrm{GeV}]$

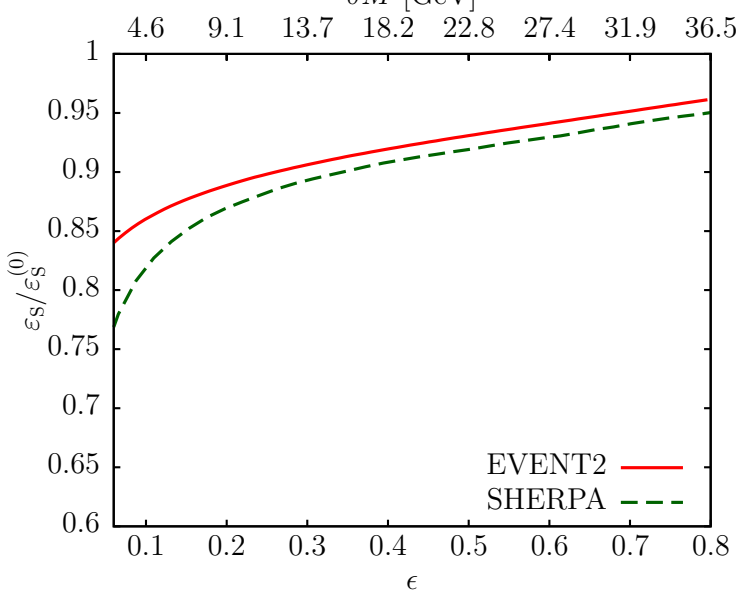

SHERPA shower vs EVENT2: Pruning

$\delta M[\mathrm{GeV}]$

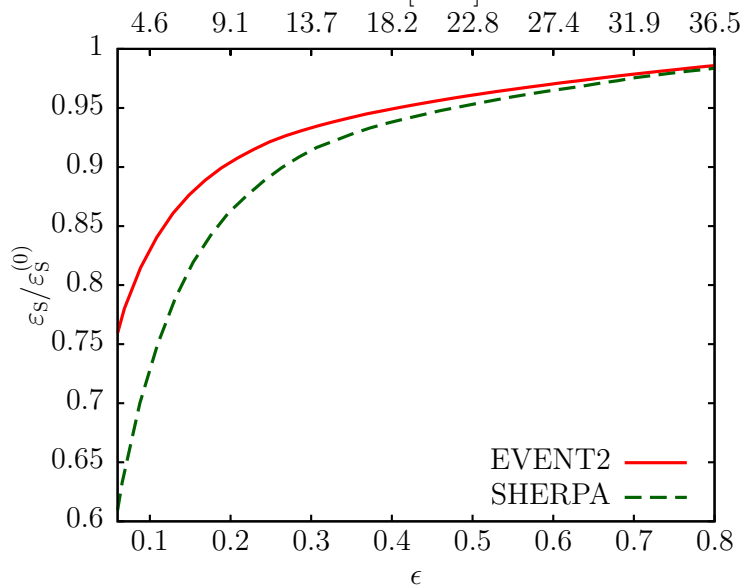

Figure 20. Ratio for signal efficiency normalised to lowest-order result, with EVENT2 and Sherpa 2.0.0, for $e^{+} e^{-}$annihilation with virtual $\mathrm{Z}$ production and hadronic decay, where we consider a $\mathrm{Z}$ boson with a transverse boost to $p_{T}=3 \mathrm{TeV}$.

To test this we ideally need to carry out an exact order $\alpha_{s}$ calculation for the process $H \rightarrow b \bar{b} g$. Such a calculation can be straightforwardly performed by taking the exact $H \rightarrow$ $b \bar{b} g$ matrix element and integrating over phase space after application of cuts corresponding to jet finding and tagging in various algorithms. While straightforward this exercise proves cumbersome and has in any case to be carried out with numerical integration. One may instead try to obtain the same information more economically by exploiting existing fixedorder codes.

One of the most reliable and long-standing fixed-order programs available to us is the code EVENT2 [66] for $e^{+} e^{-}$annihilation. We can exploit this program by considering the process $e^{+} e^{-} \rightarrow Z^{0} \rightarrow q \bar{q}$ at lowest order and with an extra gluon emission i.e. up to order $\alpha_{s}$. One can perform a boost such that the $Z^{0}$ is produced with a large momentum along a given direction and then its decay products will, a significant fraction of the time, form a single fat jet. One can then apply the boosted object taggers to tag the $Z$ boson imposing a mass window requirement $\delta M$, around $M_{Z}$ as we have done throughout this paper, for the Higgs boson. The situation is similar but not identical to the case of the Higgs we have thus far considered, due to the polarisation of the $Z$ boson so that the matrix element for $Z$ decay to quarks differs from Higgs case and efficiencies at tree-level and beyond are affected, giving for example a different dependence on $z_{\text {cut }}, y_{\text {cut }}$ at lowestorder. Nevertheless all of our conclusions about radiative corrections apply to this case as well, including our findings about the logarithmic structure of FSR contributions, since these results follow from the radiation of a gluon from the $q \bar{q}$ pair, which is given by a process independent antenna pattern, that factorises from the process dependent lowest order decay of a scalar (i.e. Higgs) or a $Z$ boson.

Therefore in order to test our basic notion that fixed-order calculations should give a comparable FSR contribution to tagging efficiency, to that from MC event generators, it 
SHERPA shower vs EVENT2 Difference: Pruning

SHERPA shower vs EVENT2 Difference: Trimming
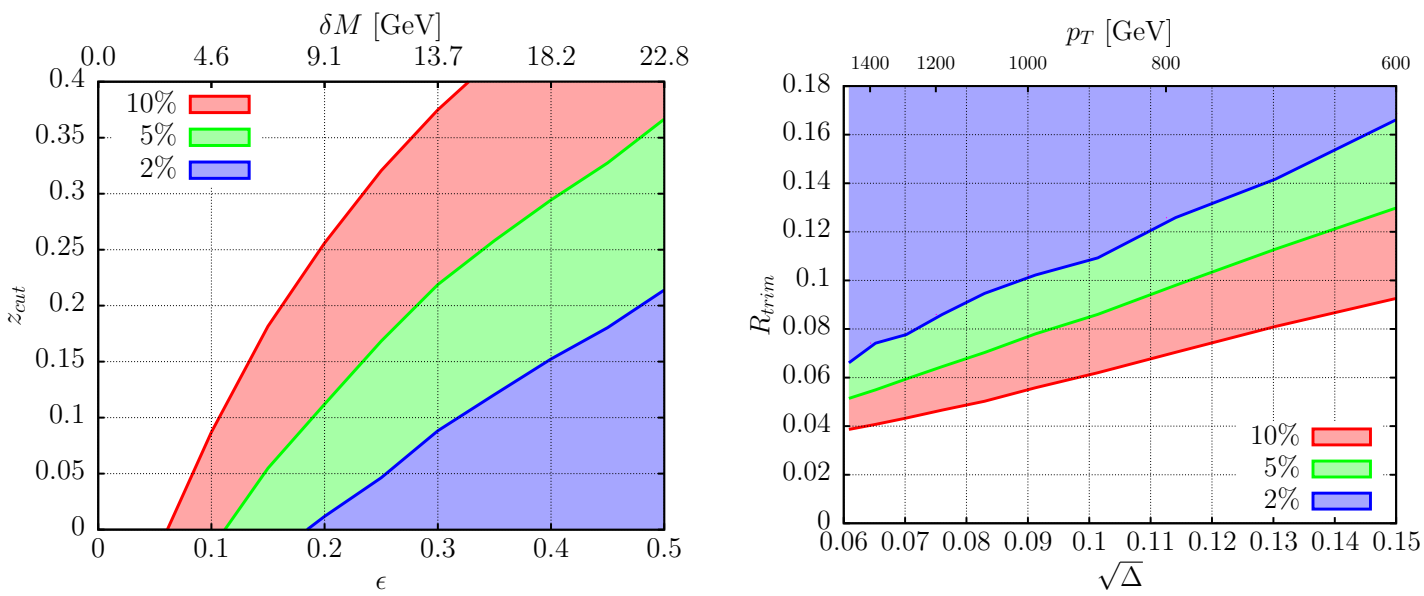

Figure 21. Contour plot showing the maximum percentage difference in signal efficiency between EVENT2 at order $\alpha_{s}$ and Sherpa 2.0.0 final state shower both normalised to the lowest order result. In the left hand panel we apply pruning with different values for $\epsilon$ and $z_{\text {cut }}$ with $p_{T}=3 \mathrm{TeV}$. In the right hand panel we apply trimming with different values for $\sqrt{\Delta}$ and $R_{\text {trim }}$ with $f_{\text {cut }}=0.1$.

should suffice to study results for boosted $Z$ bosons from EVENT2 on the one hand and MC on the other. In order to minimise any process dependence one should choose precisely the same hard process for both the fixed-order and MC and hence we choose to study the virtual $Z$ boson contribution in $e^{+} e^{-} \rightarrow q \bar{q}$ events, with the hadronically decaying $Z$ boosted to $3 \mathrm{TeV}$ (as in the EVENT2 case) with the MC generator Sherpa 2.0.0 shower [67].

We first study the signal efficiencies, normalised to the lowest order result, that are obtained with EVENT2 and Sherpa 2.0.0 for mMDT and pruning for $y_{\text {cut }}=z_{\text {cut }}=0.1$. These are shown in figure 20 as a function of the mass window, where $\epsilon=2 \delta M / M_{Z}$ as in the main text.

A first observation is that there is a reasonable degree of qualitative and quantitative similarity between LO and shower estimates, over a wide range of mass windows, which establishes further our point about the essential perturbative stability of taggers against FSR corrections. The difference between the normalised signal efficiencies for SHERPA and EVENT2 are $2 \%$ or less when $\delta M$ is greater than $\sim 8 \mathrm{GeV}$ or $\sim 13 \mathrm{GeV}$ for mMDT and pruning respectively. One should not in any case consider mass windows significantly lower than these values at high $p_{T}$, in order to minimise NP hadronisation corrections from ISR. Differences start to become more marked for very low mass windows in particular for pruning, signalling the need for resummation and hadronisation corrections. We have also verified that hadronisation corrections have a minimal impact above the $\delta M$ values stated above and hence basically preserve the picture one obtains already at leading-order. One can also similarly study trimming where the choice of $R_{\text {trim }}$ is additionally crucial to ensure that radiative corrections are minimised so that signal efficiency is maintained. Another way of making this comparison is provided in figure 21 where we show the difference between EVENT2 and Sherpa 2.0.0 efficiencies (normalised to the lowest order result) as a function of $z_{\text {cut }}$ and $\epsilon$ for pruning and as a function of $R_{\text {trim }}$ and $\Delta$ for trimming. The values of $\delta M$, 
corresponding to the $\epsilon=2 \delta M / M_{Z}$ values, are shown on the upper axis for pruning and values for $p_{T}$ corresponding to $\Delta$ are shown for trimming. The blue shaded region in each case represents parameter values where the difference between the normalised signal efficiencies for Sherpa 2.0.0 and EVENT2 are less than two percent while the green and pink regions correspond to less than five and ten percent respectively. From the plot for pruning one notes that there is a correlation between values of $\epsilon$ and $z_{\text {cut }}$ needed to minimise radiative corrections. As one goes up in $z_{\text {cut }}$, to stay within say the five percent zone, one has to correspondingly increase the size of the window. This is also in accordance with expectations from our simple analytics where one can expect large radiative corrections for $\epsilon \ll z_{\text {cut }}$.

Also, for trimming, one may expect a correlation between the value of $\Delta$ and the value of $R_{\text {trim }}$ required to minimise radiative degradation of mass. This is also reflected in figure 21 where once again the green and pink shaded regions represent differences of 5 percent and 10 percent respectively, for the normalised signal efficiencies. For $p_{T}=600 \mathrm{GeV}$, for example, choosing $R_{\text {trim }} \approx 0.13$ or larger gives less than 5 percent difference between leading order and shower descriptions. As one lowers $R_{\text {trim }}$ radiative losses get progressively larger.

Open Access. This article is distributed under the terms of the Creative Commons Attribution License (CC-BY 4.0), which permits any use, distribution and reproduction in any medium, provided the original author(s) and source are credited.

\section{References}

[1] M.H. Seymour, Searches for new particles using cone and cluster jet algorithms: a comparative study, Z. Phys. C 62 (1994) 127 [InSPIRE].

[2] J.M. Butterworth, B.E. Cox and J.R. Forshaw, WW scattering at the CERN LHC, Phys. Rev. D 65 (2002) 096014 [hep-ph/0201098] [INSPIRE].

[3] J.M. Butterworth, A.R. Davison, M. Rubin and G.P. Salam, Jet substructure as a new Higgs search channel at the LHC, Phys. Rev. Lett. 100 (2008) 242001 [arXiv:0802.2470] [INSPIRE].

[4] ATLAS collaboration, Jet mass and substructure of inclusive jets in $\sqrt{s}=7 \mathrm{TeV} p p$ collisions with the ATLAS experiment, JHEP 05 (2012) 128 [arXiv:1203.4606] [INSPIRE].

[5] ATLAS collaboration, ATLAS measurements of the properties of jets for boosted particle searches, Phys. Rev. D 86 (2012) 072006 [arXiv:1206.5369] [InSPIRE].

[6] ATLAS collaboration, Performance of jet substructure techniques for large- $R$ jets in proton-proton collisions at $\sqrt{s}=7 \mathrm{TeV}$ using the ATLAS detector, JHEP 09 (2013) 076 [arXiv: 1306.4945] [INSPIRE].

[7] CMS collaboration, Studies of jet mass in dijet and $W / Z+$ jet events, JHEP 05 (2013) 090 [arXiv: 1303.4811] [INSPIRE].

[8] ATLAS collaboration, Search for resonances decaying into top-quark pairs using fully hadronic decays in pp collisions with ATLAS at $\sqrt{s}=7$ TeV, JHEP 01 (2013) 116 [arXiv: 1211.2202] [INSPIRE].

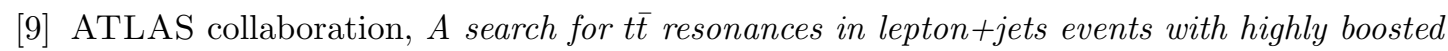
top quarks collected in pp collisions at $\sqrt{s}=7 \mathrm{TeV}$ with the ATLAS detector, JHEP 09 (2012) 041 [arXiv:1207.2409] [INSPIRE]. 
[10] ATLAS collaboration, Search for pair production of massive particles decaying into three quarks with the ATLAS detector in $\sqrt{s}=7 \mathrm{TeV}$ pp collisions at the LHC, JHEP 12 (2012) 086 [arXiv: 1210.4813] [INSPIRE].

[11] ATLAS collaboration, Search for pair-produced massive coloured scalars in four-jet final states with the ATLAS detector in proton-proton collisions at $\sqrt{s}=7 \mathrm{TeV}$, Eur. Phys. J. C 73 (2013) 2263 [arXiv:1210.4826] [INSPIRE].

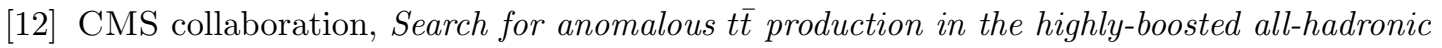
final state, JHEP 09 (2012) 029 [Erratum ibid. 03 (2014) 132] [arXiv:1204.2488] [INSPIRE].

[13] CMS collaboration, Search for resonant $t \bar{t}$ production in lepton $+j e t s$ events in pp collisions at $\sqrt{s}=7 \mathrm{TeV}$, JHEP 12 (2012) 015 [arXiv: 1209.4397] [INSPIRE].

[14] CMS collaboration, Search for heavy resonances in the $W / Z$-tagged dijet mass spectrum in pp collisions at $7 \mathrm{TeV}$, Phys. Lett. B 723 (2013) 280 [arXiv:1212.1910] [INSPIRE].

[15] G. Brooijmans, High $p_{T}$ hadronic top quark identification, ATL-PHYS-CONF-2008-008, CERN, Geneva Switzerland (2008).

[16] CMS collaboration, Search for high mass tt resonances in the all-hadronic mode, CMS-PAS-EXO-09-002, CERN, Geneva Switzerland (2009).

[17] D.E. Kaplan, K. Rehermann, M.D. Schwartz and B. Tweedie, Top tagging: a method for identifying boosted hadronically decaying top quarks, Phys. Rev. Lett. 101 (2008) 142001 [arXiv: 0806.0848] [INSPIRE].

[18] T. Plehn, M. Spannowsky, M. Takeuchi and D. Zerwas, Stop reconstruction with tagged tops, JHEP 10 (2010) 078 [arXiv: 1006. 2833] [InSPIRE].

[19] S.D. Ellis, C.K. Vermilion and J.R. Walsh, Techniques for improved heavy particle searches with jet substructure, Phys. Rev. D 80 (2009) 051501 [arXiv:0903.5081] [INSPIRE].

[20] S.D. Ellis, C.K. Vermilion and J.R. Walsh, Recombination algorithms and jet substructure: pruning as a tool for heavy particle searches, Phys. Rev. D 81 (2010) 094023 [arXiv: 0912.0033] [INSPIRE].

[21] D. Krohn, J. Thaler and L.-T. Wang, Jet trimming, JHEP 02 (2010) 084 [arXiv:0912.1342] [INSPIRE].

[22] L.G. Almeida, S.J. Lee, G. Perez, G. Sterman and I. Sung, Template overlap method for massive jets, Phys. Rev. D 82 (2010) 054034 [arXiv:1006.2035] [INSPIRE].

[23] L.G. Almeida, O. Erdogan, J. Juknevich, S.J. Lee, G. Perez and G. Sterman, Three-particle templates for a boosted Higgs boson, Phys. Rev. D 85 (2012) 114046 [arXiv:1112.1957] [INSPIRE].

[24] S.D. Ellis, A. Hornig, T.S. Roy, D. Krohn and M.D. Schwartz, Qjets: a non-deterministic approach to tree-based jet substructure, Phys. Rev. Lett. 108 (2012) 182003 [arXiv: 1201.1914] [INSPIRE].

[25] D.E. Soper and M. Spannowsky, Finding physics signals with shower deconstruction, Phys. Rev. D 84 (2011) 074002 [arXiv:1102.3480] [INSPIRE].

[26] A. Abdesselam et al., Boosted objects: a probe of beyond the standard model physics, Eur. Phys. J. C 71 (2011) 1661 [arXiv:1012.5412] [INSPIRE].

[27] A. Altheimer et al., Jet substructure at the Tevatron and LHC: new results, new tools, new benchmarks, J. Phys. G 39 (2012) 063001 [arXiv:1201.0008] [INSPIRE].

[28] A. Altheimer et al., Boosted objects and jet substructure at the LHC. Report of BOOST2012, held at IFIC Valencia, $23^{\text {rd }}-27^{\text {th }}$ of July 2012, Eur. Phys. J. C 74 (2014) 2792 [arXiv: 1311.2708] [INSPIRE]. 
[29] G.P. Salam, Towards jetography, Eur. Phys. J. C 67 (2010) 637 [arXiv:0906.1833] [INSPIRE].

[30] A.J. Larkoski, G.P. Salam and J. Thaler, Energy correlation functions for jet substructure, JHEP 06 (2013) 108 [arXiv: 1305.0007] [INSPIRE].

[31] M. Dasgupta, A. Fregoso, S. Marzani and G.P. Salam, Towards an understanding of jet substructure, JHEP 09 (2013) 029 [arXiv: 1307.0007] [INSPIRE].

[32] M. Dasgupta, A. Fregoso, S. Marzani and A. Powling, Jet substructure with analytical methods, Eur. Phys. J. C 73 (2013) 2623 [arXiv:1307.0013] [INSPIRE].

[33] A.J. Larkoski, S. Marzani, G. Soyez and J. Thaler, Soft drop, JHEP 05 (2014) 146 [arXiv: 1402.2657] [INSPIRE].

[34] M. Rubin, Non-global logarithms in filtered jet algorithms, JHEP 05 (2010) 005 [arXiv: 1002 .4557] [INSPIRE].

[35] I. Feige, M.D. Schwartz, I.W. Stewart and J. Thaler, Precision jet substructure from boosted event shapes, Phys. Rev. Lett. 109 (2012) 092001 [arXiv:1204.3898] [INSPIRE].

[36] A. Banfi and J. Cancino, Implications of QCD radiative corrections on high- $p_{T}$ Higgs searches, Phys. Lett. B 718 (2012) 499 [arXiv:1207.0674] [INSPIRE].

[37] A.J. Larkoski, I. Moult and D. Neill, Building a better boosted top tagger, Phys. Rev. D 91 (2015) 034035 [arXiv:1411.0665] [INSPIRE].

[38] M. Dasgupta, K. Khelifa-Kerfa, S. Marzani and M. Spannowsky, On jet mass distributions in $Z+j e t$ and dijet processes at the LHC, JHEP 10 (2012) 126 [arXiv:1207.1640] [INSPIRE].

[39] J.R. Forshaw, A. Kyrieleis and M.H. Seymour, Super-leading logarithms in non-global observables in QCD, JHEP 08 (2006) 059 [hep-ph/0604094] [INSPIRE].

[40] M. Dasgupta and G.P. Salam, Resummation of nonglobal QCD observables, Phys. Lett. B 512 (2001) 323 [hep-ph/0104277] [INSPIRE].

[41] R.B. Appleby and M.H. Seymour, Nonglobal logarithms in interjet energy flow with $k_{t}$ clustering requirement, JHEP 12 (2002) 063 [hep-ph/0211426] [INSPIRE].

[42] A. Banfi and M. Dasgupta, Problems in resumming interjet energy flows with $k_{t}$ clustering, Phys. Lett. B 628 (2005) 49 [hep-ph/0508159] [INSPIRE].

[43] Y. Delenda, R. Appleby, M. Dasgupta and A. Banfi, On QCD resummation with $k_{t}$ clustering, JHEP 12 (2006) 044 [hep-ph/0610242] [INSPIRE].

[44] M. Bahr et al., HERWIG++ physics and manual, Eur. Phys. J. C 58 (2008) 639 [arXiv:0803.0883] [INSPIRE].

[45] J. Bellm et al., HERWIG++ 2.7 release note, arXiv:1310.6877 [INSPIRE].

[46] M.H. Seymour and A. Siodmok, Constraining MPI models using $\sigma_{\text {eff }}$ and recent Tevatron and LHC underlying event data, JHEP 10 (2013) 113 [arXiv:1307.5015] [INSPIRE].

[47] A. Buckley et al., Rivet user manual, Comput. Phys. Commun. 184 (2013) 2803 [arXiv: 1003.0694] [INSPIRE].

[48] Y.L. Dokshitzer, G.D. Leder, S. Moretti and B.R. Webber, Better jet clustering algorithms, JHEP 08 (1997) 001 [hep-ph/9707323] [INSPIRE].

[49] M. Wobisch and T. Wengler, Hadronization corrections to jet cross-sections in deep inelastic scattering, in Monte Carlo generators for HERA physics, Hamburg Germany (1999), pg. 270 [hep-ph/9907280] [INSPIRE]. 
[50] M. Cacciari, G.P. Salam and G. Soyez, FastJet user manual, Eur. Phys. J. C 72 (2012) 1896 [arXiv: 1111.6097] [INSPIRE].

[51] S. Gieseke, C. Rohr and A. Siodmok, Colour reconnections in HERWIG++, Eur. Phys. J. C 72 (2012) 2225 [arXiv: 1206.0041] [inSPIRE].

[52] M. Bahr, M. Myska, M.H. Seymour and A. Siodmok, Extracting $\sigma_{\text {effective }}$ from the $C D F \gamma+3$ jets measurement, JHEP 03 (2013) 129 [arXiv:1302.4325] [INSPIRE].

[53] CDF, D0 collaboration, T. Aaltonen, A. Buzatu, B. Kilminster, Y. Nagai and W. Yao, Improved b-jet energy correction for $H \rightarrow b \bar{b}$ searches at $C D F$, arXiv:1107.3026 [INSPIRE].

[54] M. Dasgupta, L. Magnea and G.P. Salam, Non-perturbative QCD effects in jets at hadron colliders, JHEP 02 (2008) 055 [arXiv:0712.3014] [INSPIRE].

[55] G. Soyez, G.P. Salam, J. Kim, S. Dutta and M. Cacciari, Pileup subtraction for jet shapes, Phys. Rev. Lett. 110 (2013) 162001 [arXiv:1211.2811] [INSPIRE].

[56] D. Krohn, M.D. Schwartz, M. Low and L.-T. Wang, Jet cleansing: pileup removal at high luminosity, Phys. Rev. D 90 (2014) 065020 [arXiv:1309.4777] [INSPIRE].

[57] M. Cacciari and G.P. Salam, Pileup subtraction using jet areas, Phys. Lett. B 659 (2008) 119 [arXiv: 0707.1378] [INSPIRE].

[58] P. Richardson and D. Winn, Investigation of Monte Carlo uncertainties on Higgs boson searches using jet substructure, Eur. Phys. J. C 72 (2012) 2178 [arXiv:1207.0380] [INSPIRE].

[59] A. Buckley et al., General-purpose event generators for LHC physics, Phys. Rept. 504 (2011) 145 [arXiv: 1101.2599] [INSPIRE].

[60] S.D. Ellis and D.E. Soper, Successive combination jet algorithm for hadron collisions, Phys. Rev. D 48 (1993) 3160 [hep-ph/9305266] [INSPIRE].

[61] J. Thaler and L.-T. Wang, Strategies to identify boosted tops, JHEP 07 (2008) 092 [arXiv: 0806.0023] [INSPIRE].

[62] J. Thaler and K. Van Tilburg, Identifying boosted objects with $N$-subjettiness, JHEP 03 (2011) 015 [arXiv: 1011.2268] [INSPIRE].

[63] M. Dasgupta, A. Powling and A. Siodmok, work in progress.

[64] D.E. Soper and M. Spannowsky, Combining subjet algorithms to enhance ZH detection at the LHC, JHEP 08 (2010) 029 [arXiv: 1005. 0417] [INSPIRE].

[65] G. Soyez, Theory lessons from LHC Run I, talk delivered at BOOST 2014, http://www.hep.ucl.ac.uk/boost2014, University College London, London U.K. August 18-22 2014.

[66] S. Catani and M.H. Seymour, The dipole formalism for the calculation of QCD jet cross-sections at next-to-leading order, Phys. Lett. B 378 (1996) 287 [hep-ph/9602277] [INSPIRE].

[67] T. Gleisberg et al., Event generation with SHERPA 1.1, JHEP 02 (2009) 007 [arXiv:0811.4622] [INSPIRE].

[68] T. Sjöstrand, S. Mrenna and P.Z. Skands, PYTHIA 6.4 physics and manual, JHEP 05 (2006) 026 [hep-ph/0603175] [INSPIRE].

[69] CDF collaboration, T. Aaltonen et al., Studying the underlying event in Drell-Yan and high transverse momentum jet production at the Tevatron, Phys. Rev. D 82 (2010) 034001 [arXiv: 1003.3146] [INSPIRE]. 